M. Ueda

Nagoya Math. J.

Vol. 149 (1998), 117-171

\title{
ON TWISTING OPERATORS AND NEWFORMS OF HALF-INTEGRAL WEIGHT II COMPLETE THEORY OF NEWFORMS FOR KOHNEN SPACE
}

\author{
MASARU UEDA ${ }^{1}$
}

\begin{abstract}
The author continues his previous work with the purpose of establishing a theory of newforms in the case of half-integral weight. In this paper, the author formulates and proves a complete theory of newforms for Kohnen space. Kohnen space is an important canonical subspace in the space of cusp forms of half-integral weight $k+1 / 2(k>0)$. Every Hecke common eigenform in Kohnen space corresponds to a primitive form of integral weight $2 k$ and of odd level via Shimura Correspondence.

These newforms for Kohnen space satisfy the Strong multiplicity One theorem. Moreover, we explicitly determine the corresponding primitive form (of weight $2 k$ ) to each newform for Kohnen space. The space of oldforms is also explicitly described.

In order to find all newforms for Kohnen space, the author needs a certain non-vanishing property of Fourier coefficients of cusp forms. Such property proves by using representation theory of finite linear groups. The method of proof of newform theory is mainly based on trace formulae and trace relations.
\end{abstract}

\section{Introduction}

The theory of newforms is very important and useful for arithmetical study of modular forms of integral weight. This theory has the following nice properties:

(i) The space of newforms have an orthogonal C-basis consisting of common eigenforms for all Hecke operators and such common eigenforms are uniquely determined up to multiplication of complex numbers. Moreover such common eigenforms satisfy the Strong Multiplicity One Theorem (cf. $[\mathrm{M}, \S 4.6])$.

\footnotetext{
Received June 13, 1996.

${ }^{1}$ Partly supported by the Grants-in Aid for Scientific Research, the Ministry of Education of Japan.
} 
(ii) The full space of cusp forms, $S(2 k, N)$, can be reconstructed by the space of newforms, i.e., we have the following decomposition:

$$
S(2 k, N)=\bigoplus_{0<B \mid N} \bigoplus_{0<A \mid(N / B)} S^{0}(2 k, B) \mid\left[\left(\begin{array}{ll}
A & 0 \\
0 & 1
\end{array}\right)\right]_{2 k}
$$

where $S^{0}(2 k, B)$ is the space of newforms (see below $\S 0$ and [M, $\left.\S 4.6\right]$ ).

(iii) The above operator $f \rightarrow f \mid\left[\left(\begin{array}{ll}A & 0 \\ 0 & 1\end{array}\right)\right]_{2 k}$ (almost) preserves the Fourier coefficients of the cusp form. Hence for studying the Fourier coefficients of cusp forms, it is sufficient to study cusp forms only in the space of newforms.

(iv) The theory of newforms has tight relations to both Representation theory and Geometry.

Until now, several authors have attempted to find a similar theory of newforms of half-integral weight which satisfy similar properties like the above (i)-(iv) (cf. [She], [N], [K], [M-R-V], [U1], [She-W]).

In the paper $[\mathrm{K}], \mathrm{W}$. Kohnen defined (what is called) Kohnen space which can be considered as the canonical subspace corresponding to cusp forms of integral weight and of odd level via Shimura correspondence. And when the level is a $4 \times$ (odd squarefree integer), he also established a theory of newforms for this Kohnen space.

In the previous paper [U1], the author generalized Kohnen's work and obtained a similar theory of newforms for Kohnen space of arbitrary level $(=4 \times$ (odd integer), cf. [U1, §3]). But those results are half-way for a technical reason (cf. [U1, §4]).

The aim of this paper is to complete the results in the previous paper and to formulate and state a complete theory of newforms of Kohnen space.

Let us precisely state a formulation of the theory of newforms for Kohnen space.

Let $k, N \in \mathbf{Z}_{+}$and $N$ divisible by 4 . Let $\chi$ be an even character modulo $N$ with $\chi^{2}=1$. We denote the $p$-adic additive valuation for any integer $m$ by $\operatorname{ord}_{p}(m)$. We decompose $N$ as follows:

$$
N=2^{\operatorname{ord}_{2}(N)} M_{1} M_{2+}, \quad M_{1}:=\prod_{\substack{p \mid N, p \neq 2 \\ \operatorname{ord} p(N)=1}} p, \quad M_{2+}:=\prod_{\substack{p \mid N, p \neq 2 \\ \operatorname{ord} p(N) \geq 2}} p^{\operatorname{ord}_{p}(N)} .
$$

Denote the set of all prime divisors of $M_{2+}$ by $\Pi$. 
Kohnen spaces $S(k+1 / 2, N, \chi)_{K}$ can be defined only in the case of $\operatorname{ord}_{2}(N)=2$ (See below $\S 0(\mathrm{~d})$ for the definition). Put $M=M_{1} M_{2+}$.

We shall consider such a case. For simplifying the explanation, we deal with only the case of $k \geq 2$. In the case of $k=1$, we must slightly modify subspaces (cf. §0).

Define the space of oldform $\mathfrak{O}(k+1 / 2, N, \chi)_{K}$ as follows.

$$
\begin{aligned}
\mathfrak{O}(k+1 / 2, N, \chi)_{K} & =\sum_{\substack{0<B \mid M \\
B \neq M}} \sum_{\substack{0<A \mid(M / B) \\
\xi(\underline{A})=\chi}} S(k+1 / 2,4 B, \xi)_{K} \mid \tilde{\delta}_{A} \\
& +\sum_{\substack{0<B \mid M \\
B \neq M}} \sum_{\substack{0<A \mid(M / B)^{2} \\
\xi(\underline{A})=\chi}} \sum_{\substack{0 \leq e_{l} \leq 2 \\
(l \in \Pi)}} S(k+1 / 2,4 B, \xi)_{K} \mid U(A) \prod_{l \in \Pi} R_{l} e_{l} .
\end{aligned}
$$

Here, $\xi$ runs over all characters modulo $4 B$ such that $\xi(\underline{A})=\chi$. The operator $\tilde{\delta}_{A}$, the shift operator $U(A)$, and the twisting operator $R_{l}(l \in \Pi)$ are defined as follows: For $f=\sum_{n \geq 1} a(n) \mathbf{e}(n z)$,

$$
\begin{aligned}
f \mid \tilde{\delta}_{A}(z) & :=A^{k / 2+1 / 4} f(A z)=A^{k / 2+1 / 4} \sum_{n \geq 1} a(n) \mathbf{e}(A n z), \\
f \mid U(A)(z) & :=\sum_{n \geq 1} a(A n) \mathbf{e}(n z), \\
f \mid R_{l}(z) & :=\sum_{n \geq 1} a(n)\left(\frac{n}{l}\right) \mathbf{e}(n z) .
\end{aligned}
$$

The space $\mathfrak{O}(k+1 / 2, N, \chi)_{K}$ is a subspace of $S(k+1 / 2, N, \chi)_{K}$. We denote by $\mathfrak{N}(k+1 / 2, N, \chi)_{K}$ the orthogonal complement of $\mathfrak{O}(k+1 / 2, N, \chi)_{K}$ in $S(k+1 / 2, N, \chi)_{K}$.

The space $\mathfrak{N}(k+1 / 2, N, \chi)_{K}$ is stable by the twisting operators $R_{p}$ for all $p \in \Pi$. Hence we can decompose this space into common eigen subspaces as follows:

$$
\begin{gathered}
\mathfrak{N}(k+1 / 2, N, \chi)_{K}=\oplus_{\kappa \in\{ \pm 1\} \Pi} \mathfrak{N}^{\emptyset, \kappa}(k+1 / 2, N, \chi)_{K} \\
\mathfrak{N}^{\emptyset, \kappa}(k+1 / 2, N, \chi)_{K}:=\left\{f \in \mathfrak{N}(k+1 / 2, N, \chi)_{K} ;\right. \\
\left.f \mid R_{p}=\kappa(p) f \text { for all } p \in \Pi\right\} .
\end{gathered}
$$

Here $\{ \pm 1\}^{\Pi}:=\operatorname{Map}(\Pi,\{ \pm 1\})$. 
We call these spaces $\mathfrak{N}^{\emptyset, \kappa}(k+1 / 2, N, \chi)_{K}\left(\kappa \in\{ \pm 1\}^{\Pi}\right)$ the spaces of newforms, because these subspaces have the following nice properties (cf. below $\S 3$ and especially Theorem 4).

(1) $\mathfrak{N}^{\emptyset, \kappa}(k+1 / 2, N, \chi)_{K}$ has an orthogonal $\mathbf{C}$-basis consisting of common eigenforms for all Hecke operators $\tilde{T}_{k+1 / 2, N, \chi}\left(p^{2}\right)(p$ : prime, $p \nmid \backslash M)$ and $U\left(p^{2}\right)$ ( $p$ : prime, $\left.p \mid M\right)$, which are uniquely determined up to multiplication with non-zero complex numbers. Let $f$ be such a common eigenform and $\lambda_{p}$ the eigenvalue of $f$ with respect to $\tilde{T}_{k+1 / 2, N, \chi}\left(p^{2}\right)(p \nmid M)$ resp. $U\left(p^{2}\right)(p \mid M)$. Then there exists a primitive form $F \in S^{0}(2 k, M)$ of weight $2 k$ and of conductor $M$ which is uniquely determined and satisfies the following: For a prime $p$,

$$
F \mid T_{2 k, M}(p)=\lambda_{p} F \quad \text { if }(p, M)=1 \quad \text { and } \quad F \mid U(p)=\lambda_{p} F \quad \text { if } p \mid M
$$

Furthermore we can find, by using the trace relation of Theorem 2 (1), which primitive form occurs via the above correspondence.

\section{(2) (The Strong Multiplicity One Theorem)}

Let $f, g$ be two non-zero elements of $\mathfrak{N}^{\emptyset, \kappa}(k+1 / 2, N, \chi)_{K}$. If $f$ and $g$ are common eigenforms of $\tilde{T}_{k+1 / 2, N, \chi}\left(p^{2}\right)$ with the same eigenvalue for all prime numbers $p$ prime to some integer $A$, then $\mathbf{C} f=\mathbf{C} g$.

Therefore $\mathfrak{N}^{\emptyset, \kappa}(k+1 / 2, N, \chi)_{K} \hookrightarrow S^{0}(2 k, M)$ as modules on the full Hecke algebra.

(3) The space of oldforms $\mathfrak{O}(k+1 / 2, N, \chi)_{K}$ has also an orthogonal C-basis consisting of common eigenforms for all operators $\tilde{T}_{k+1 / 2, N, \chi}\left(p^{2}\right)$ ( $p$ : prime, $p \nmid N)$. The system of eigenvalues of such a common eigenform corresponds to a primitive form of weight $2 k$ whose conductor is a divisor of $M$ and is less than $M$ (cf. Theorem 3).

(4) The space of oldform $\mathfrak{O}(k+1 / 2, N, \chi)_{K}$ is generated by the spaces of cusp forms of lower level. Hence, by induction, we see that the spaces $S(k+1 / 2, N, \chi)_{K}$ are reconstructed by the spaces of the types of $\mathfrak{N}^{\emptyset, \kappa}(k+$ $1 / 2,4 B, \xi)_{K}$ and the operators of the types of $\tilde{\delta}_{A}, U(A)$, and $R_{l}$.

From the above definition these operators $\tilde{\delta}_{A}, U(A)$, and $R_{l}$ (almost) preserve Fourier coefficients of cusp forms. Hence for studying Fourier coefficients of cusp forms $\in S(k+1 / 2, N, \chi)_{K}$, it is sufficient to study cusp forms only in the spaces of newforms $\mathfrak{N}^{\emptyset, \kappa}(k+1 / 2, N, \chi)_{K}$.

Finally the author has some comments. 
There exists a case such that $\mathfrak{N}^{\emptyset, \kappa}(k+1 / 2, N, \chi)_{K} \cong \mathfrak{N}^{\emptyset, \kappa^{\prime}}(k+1 / 2, N, \chi)_{K}$ as modules on Hecke algebra for two distinct $\kappa, \kappa^{\prime} \in\{ \pm 1\}^{\Pi}$. See the formula (3.4) for such an example.

It seems likely that there exists a similar theory for any full spaces of cusp forms $S(k+1 / 2, N, \chi)$ even if $\operatorname{ord}_{2}(N) \geq 3$.

In the case of $\operatorname{ord}_{2}(N) \leq 3$, the author thinks that necessary preparations have already done in the author's previous papers [U3-5]. But situations are quite different in the case of $\operatorname{ord}_{2}(N) \geq 4$ (cf. [U5]).

It seems that its reason is the existence of the twisting operators for characters $(-\underline{-1})$ and $(\underline{2})$. These twisting operators can be defined only if $\operatorname{ord}_{2}(N) \geq 4$ and $\operatorname{ord}_{2}(N) \geq 6$, respectively. See the forthcoming papers [U6].

This paper is composed as follows: $\S 0$ is general preliminaries. $\S 1$ is preparation from representation theory of finite groups. We apply results of $\S 1$ to calculations of $\S 2$. In $\S 2$, we will complete an attempt in the previous paper [U1, §4]. §3 is the main part of this paper. We will formulate and state a complete theory of newforms for Kohnen space in $\S 3$. In the Appendix, we will prove several general formulae which are used in calculations of $\S 2$.

The author wrote this paper while he was staying at Max-PlanckInstitut. The author would like to express his hearty thanks to Max-PlanckInstitut and its staff for their warm hospitality.

\section{§o. Notational Preliminary}

Throughout this paper, we use the following notations.

(a) General notations.

Let $A, B$ be subsets of a set $X$ and $\left\{A_{i}\right\}_{i \in I}$ a family of subsets of $X$. If $A \cup B$ is a disjoint union, then we denote $A+B:=A \cup B$ for simplicity. Similarly, if $\bigcup_{i \in I} A_{i}$ is a disjoint union, then we denote $\sum_{i \in I} A_{i}:=\bigcup_{i \in I} A_{i}$.

We denote the set of positive integers by $\mathbf{Z}_{+}$. We denote the additive valuation for any integer $m$ by $\operatorname{ord}_{p}(m)$.

See $[\mathrm{M}, \mathrm{p} .82]$ for the definition of the Kronecker symbol $\left(\frac{a}{b}\right)(a, b$ integers with $(a, b) \neq(0,0))$.

Let $N$ be a positive integer and $m$ an integer $\neq 0$. We write $m \mid N^{\infty}$ if every prime factor of $m$ divides $N$.

Let $k$ denote a non-negative integer. If $z \in \mathbf{C}$ and $x \in \mathbf{C}$, we put $z^{x}=\exp (x \cdot \log (z))$ with $\log (z)=\log (|z|)+\sqrt{-1} \arg (z), \arg (z)$ being 
determined by $-\pi<\arg (z) \leq \pi$. Also we put $\mathbf{e}(z)=\exp (2 \pi \sqrt{-1} z)$.

Let $\mathfrak{H}$ be the complex upper half plane. For a complex-valued function $f(z)$ on $\mathfrak{H}, \alpha=\left(\begin{array}{ll}a & b \\ c & d\end{array}\right) \in G L_{2}^{+}(\mathbf{R}), \gamma=\left(\begin{array}{ll}u & v \\ w & x\end{array}\right) \in \Gamma_{0}(4)$ and $z \in \mathfrak{H}$, we define functions $J(\alpha, z), j(\gamma, z)$ and $f \mid[\alpha]_{k}(z)$ on $\mathfrak{H}$ by: $J(\alpha, z)=c z+d, j(\gamma, z)=$ $\left(\frac{-1}{x}\right)^{-1 / 2}\left(\frac{w}{x}\right)(w z+x)^{1 / 2}$ and $f \mid[\alpha]_{k}(z)=(\operatorname{det} \alpha)^{k / 2} J(\alpha, z)^{-k} f(\alpha z)$.

For $m \in \mathbf{Z}_{+}$we define a shift operator $U(m)$ on formal power series in $\mathbf{e}(z)$ by

$$
\sum_{n \geq 0} a(n) \mathbf{e}(n z) \mid U(m):=\sum_{n \geq 0} a(m n) \mathbf{e}(n z) .
$$

Let $\chi$ be a Dirichlet character modulo $N$. We denote the conductor of $\chi$ by $f(\chi)$ and the $p$-primary component of $\chi$ by $\chi_{p}$ for each prime divisor $p$ of $N$.

Let $V, V^{\prime}$ be finite-dimensional vector spaces over $\mathbf{C}$. We denote the trace of a linear operator $T$ on $V$ by $\operatorname{tr}(T ; V)$ and also the kernel of a linear map $F$ from $V$ to $V^{\prime}$ by $\operatorname{Ker}(F ; V)$.

We denote the set of all mapping from a set $A$ to a set $B$ by $\operatorname{Map}(A, B)$. Furthermore we use the abbreviated notation $B^{A}(=\operatorname{Map}(A, B))$.

Let $A$ be a set of prime numbers and $\left(\alpha_{p}\right)_{p \in A}$ a system of integers. We put the following notation: $A(\alpha)_{i}:=\left\{p \in A \mid \alpha_{p}=i\right\}$ and $A(\alpha)_{i+}:=\{p \in$ $\left.A \mid \alpha_{p} \geq i\right\}$ for any $i \in \mathbf{Z}$.

(b) Modular forms of integral weight.

Let $k$ and $N$ be positive integers. By $S(2 k, N)$, we denote the space of all holomorphic cusp forms of weight $2 k$ with the trivial character on the group $\Gamma=\Gamma_{0}(N)$. We also denote the subspace of $S(2 k, N)$ spanned by all newforms in $S(2 k, N)$ by $S^{0}(2 k, N)$.

Let $\alpha \in G L_{2}^{+}(\mathbf{R})$. If $\Gamma$ and $\alpha^{-1} \Gamma \alpha$ are commensurable, we define a linear operator $[\Gamma \alpha \Gamma]_{2 k}$ on $S(2 k, N)$ by: $f\left|[\Gamma \alpha \Gamma]_{2 k}=(\operatorname{det} \alpha)^{k-1} \sum_{\alpha_{i}} f\right|$ $\left[\alpha_{i}\right]_{2 k}$, where $\alpha_{i}$ runs over a system of representatives for $\Gamma \backslash \Gamma \alpha \Gamma$. For a positive integer $n$ with $(n, N)=1$, we put $T_{2 k, N}(n)=\sum_{a d=n}\left[\Gamma\left(\begin{array}{ll}a & 0 \\ 0 & d\end{array}\right) \Gamma\right]_{2 k}$, where the sum is extended over all pairs of integers $(a, d)$ such that $a, d>0$, $a \mid d, a d=n$.

Let $Q$ be a positive divisor of $N$ such that $(Q, N / Q)=1$. Take any 
element $\gamma_{Q} \in S L_{2}(\mathbf{Z})$ which satisfies the conditions:

$$
\gamma_{Q} \equiv \begin{cases}\left(\begin{array}{cc}
0 & -1 \\
1 & 0
\end{array}\right) & (\bmod Q) \\
\left(\begin{array}{ll}
1 & 0 \\
0 & 1
\end{array}\right) & (\bmod N / Q)\end{cases}
$$

Put $W(Q)=\gamma_{Q}\left(\begin{array}{ll}Q & 0 \\ 0 & 1\end{array}\right)$. The following facts are well-known: $W(Q)$ is a normalizer of $\Gamma ;[W(Q)]_{2 k}$ induces a C-linear automorphism of order 2 on $S(2 k, N)$ and this operator is independent of a choice of an element $\gamma_{Q}$. For $Q=1$, we can take $\gamma_{1}=W(1)=\left(\begin{array}{ll}1 & 0 \\ 0 & 1\end{array}\right)$. Hence we have $[W(1)]_{2 k}=$ 1. Moreover for the sake of simplicity, we use the following abbreviated notation: Let $A$ be a subset of the set of all prime divisors of $N$. Then $W_{A}:=W\left(\prod_{p \in A} p^{\operatorname{ord}_{p}(N)}\right)$. In particular, we simply write $W_{l}=W_{A}$ if $A=\{l\}$.

Moreover, if the subscripts are obvious and any confusion does not occur, we simply write $T(n)=T_{2 k, N}(n)$ and $W(Q)=[W(Q)]_{2 k}$, etc..

For any $f(z)=\sum_{n=1}^{\infty} a(n) \mathbf{e}(n z) \in S(2 k, N)$ and $\chi$ a primitive character modulo $\mathfrak{f}=\mathfrak{f}(\chi)$, put $f \mid R_{\chi}(z):=\sum_{n=1}^{\infty} \chi(n) a(n) \mathbf{e}(n z)$. From [Sh 3, Prop. 3.64] we have $f \mid R_{\chi} \in S\left(2 k, N^{\prime}, \chi^{2}\right)$, where $N^{\prime}$ is the least common multiple of $N$ and $\mathfrak{f}(\chi)^{2}$. We call this operator $R_{\chi}$ the twisting operator of $\chi$.

(c) Modular forms of half-integral weight.

Let $k$ denote a non-negative integer, $N$ a positive integer divisible by 4 , and $\chi$ an even character modulo $N$ such that $\chi^{2}=1$. Put $\mu=\operatorname{ord}_{2}(N)$, $M=2^{-\mu} N$ and $\Gamma_{0}=\Gamma_{0}(N)$. Then there is a square-free odd positive divisor $M_{0}$ of $M$ such that $\chi=\left(\frac{M_{0}}{}\right)$ or $\left(\underline{2 M_{0}}\right)$ (the Kronecker symbol).

Let $\mathfrak{G}(k+1 / 2)$ be the group consisting of all pairs $(\alpha, \varphi)$, where $\alpha=$ $\left(\begin{array}{ll}a & b \\ c & d\end{array}\right) \in G L_{2}^{+}(\mathbf{R})$ and $\varphi$ is a holomorphic function on $\mathfrak{H}$ satisfying $\varphi(z)=$ $t(\operatorname{det} \alpha)^{-k / 2-1 / 4} J(\alpha, z)^{k+1 / 2}$ with $t \in \mathbf{C}$ and $|t|=1$. The group law is defined by: $(\alpha, \varphi(z)) \cdot(\beta, \psi(z))=(\alpha \beta, \varphi(\beta z) \psi(z))$. For a complex-valued function $f$ on $\mathfrak{H}$ and $(\alpha, \varphi) \in \mathfrak{G}(k+1 / 2)$, we define a function $f \mid(\alpha, \varphi)$ on $\mathfrak{H}$ by: $f \mid(\alpha, \varphi)(z)=\varphi(z)^{-1} f(\alpha z)$. Moreover if there will be no confusion, we also write $\gamma^{*}=\left(\gamma, j(\gamma, z)^{2 k+1}\right)$ for all $\gamma \in \Gamma_{0}(4)$. 
By $\Delta_{0}=\Delta_{0}(N, \chi)=\Delta_{0}(N, \chi)_{k+1 / 2}$, we denote the subgroup of $\mathfrak{G}(k$ $1 / 2)$ consisting of all pairs $(\gamma, \varphi)$, where $\left(\begin{array}{ll}a & b \\ c & d\end{array}\right)=\gamma \in \Gamma_{0}$ and $\varphi(z)=$ $\chi(d) j(\gamma, z)^{2 k+1}$ and also denote $\Delta_{1}=\Delta_{1}(N):=\left\{\gamma^{*} \mid \gamma \in \Gamma_{1}(N)\right\}$.

We denote by $G(k+1 / 2, N, \chi)$ (resp. $S(k+1 / 2, N, \chi)$ ) the space of integral (resp. cusp) forms of weight $k+1 / 2$ with the character $\chi$ on the group $\Gamma_{0}$, namely, the space of all the complex-valued holomorphic functions $f$ on $\mathfrak{H}$ which satisfy $f \mid \xi=f$ for all $\xi \in \Delta_{0}$ and which are holomorphic (resp. are holomorphic and vanish) at all cusps of $\Gamma_{0}$. Moreover we also denote by $S\left(k+1 / 2, \Delta_{1}(N)\right)$ the space of cusp forms of weight $k+1 / 2$ on the group $\Gamma_{1}(N)$ i.e., the space of all the complex-valued holomorphic functions $f$ on $\mathfrak{H}$ which satisfy $f \mid \xi=f$ for all $\xi \in \Delta_{1}$ and which are holomorphic and vanish at all cusps of $\Gamma_{1}(N)$ ([cf. Sh 1]).

In the case $k=1$, i.e., the case of weight $3 / 2, S(3 / 2, N, \chi)$ contains theta series of special type. We know that these theta series correspond to Eisenstein series via Shimura correspondence.

From this reason we define the orthogonal complement $V(N ; \chi)$ of the space of such theta series in $S(3 / 2, N, \chi)$. In the case of weight $3 / 2$, we deal only with this complement $V(N ; \chi)$. See $[\mathrm{U} 1, \S 0, \S 1]$ for the details.

Let $\xi \in \mathfrak{G}(k+1 / 2)$. If $\Delta_{0}$ and $\xi^{-1} \Delta_{0} \xi$ are commensurable, we define a linear operator $\left[\Delta_{0} \xi \Delta_{0}\right]_{k+1 / 2}$ on $G(k+1 / 2, N, \chi)$ and $S(k+1 / 2, N, \chi)$ by: $f\left|\left[\Delta_{0} \xi \Delta_{0}\right]_{k+1 / 2}=\sum_{\eta} f\right| \eta$, where $\eta$ runs over a system of representatives for $\Delta_{0} \backslash \Delta_{0} \xi \Delta_{0}$. Similarly, if $\Delta_{1}$ and $\xi^{-1} \Delta_{1} \xi$ are commensurable, we define a linear operator $\left[\Delta_{1} \xi \Delta_{1}\right]$ on $S\left(k+1 / 2, \Delta_{1}(N)\right)$ by: $f\left|\left[\Delta_{1} \xi \Delta_{1}\right]=\sum_{\eta \in \Delta_{1} \backslash \Delta_{1} \xi \Delta_{1}} f\right| \eta$.

Then for a positive integer $n$ with $(n, N)=1$, we put

$$
\tilde{T}_{k+1 / 2, N, \chi}\left(n^{2}\right)=n^{k-3 / 2} \sum_{a d=n} a\left[\Delta_{0}\left(\left(\begin{array}{cc}
a^{2} & 0 \\
0 & d^{2}
\end{array}\right),(d / a)^{k+1 / 2}\right) \Delta_{0}\right]_{k+1 / 2},
$$

where the sum is extended over all pairs of integers $(a, d)$ such that $a$, $d>0, a \mid d$ and $a d=n$. We simply write $\tilde{T}\left(n^{2}\right):=\tilde{T}_{k+1 / 2, N, \chi}\left(n^{2}\right)$ if the subscripts are obvious and any confusion does not occur. These operators $\tilde{T}\left(n^{2}\right)((n, N)=1)$ are hermitian and commutative with each other on $S(k+1 / 2, N, \chi)$ (cf. [Sh 2, lemma 5], [Sh 3, Prop. (3.32)], [U1, (1.9)]).

For any $m \in \mathbf{Z}_{+}$, put $\tilde{\delta}_{m}:=\left(\left(\begin{array}{cc}m & 0 \\ 0 & 1\end{array}\right), m^{-k / 2-1 / 4}\right)$. 
Let $Q$ be an odd positive divisor of $N$ such that $(Q, N / Q)=1$. Take any element $\gamma_{Q} \in S L_{2}(\mathbf{Z})$ satisfying the conditions:

$$
\gamma_{Q} \equiv \begin{cases}\left(\begin{array}{cc}
0 & -1 \\
1 & 0
\end{array}\right) & (\bmod Q) \\
\left(\begin{array}{ll}
1 & 0 \\
0 & 1
\end{array}\right) & (\bmod N / Q)\end{cases}
$$

Then $\gamma_{Q} \in \Gamma_{0}(N / Q) \subseteq \Gamma_{0}(4)$. Put $\widetilde{W}(Q):=\gamma_{Q}{ }^{*} \tilde{\delta}_{Q} \in \mathfrak{G}(k+1 / 2)$. See [U1, $\S 1]$ for the details of properties of these $\tilde{\delta}_{m}$ and $\widetilde{W}(Q)$.

Let $f(z)=\sum_{n=0}^{\infty} a(n) \mathbf{e}(n z) \in G(k+1 / 2, N, \chi)$ and $\psi$ a primitive character modulo $\mathfrak{f}(\psi)$. Let $N^{\prime}$ be the least common multiple of $N, \mathfrak{f}(\psi)^{2}$, and $\mathfrak{f}(\psi) \mathfrak{f}(\chi)$. Then $f \mid R_{\psi}(z):=\sum_{n=0}^{\infty} \psi(n) a(n) \mathbf{e}(n z)$ belongs to the space $G\left(k+1 / 2, N^{\prime}, \chi \psi^{2}\right)$. In particular, if $f$ is a cusp form, so is $f \mid R_{\psi}$. [Sh 1 , Lemma 3.6]. We call this operator $R_{\psi}$ the twisting operator of $\psi$.

(d) Kohnen space.

We keep to the notations in the subsection (c).

Let $k$ be a positive integer. Suppose that $N=4 M$ and $M$ is an odd natural number. We define the Kohnen space $S(k+1 / 2, N, \chi)_{K}$ as follows:

$$
S\left(k+\frac{1}{2}, N, \chi\right)_{K}=\left\{\begin{array}{l}
S\left(k+\frac{1}{2}, N, \chi\right) \ni f(z)=\sum_{n=1}^{\infty} a(n) \mathbf{e}(n z) ; \\
a(n)=0 \text { for } \chi_{2}(-1)(-1)^{k} n \equiv 2,3 \quad(\bmod 4)
\end{array}\right\},
$$

where $\chi_{2}$ is the 2-primary component of $\chi$.

In the case of weight $3 / 2$, we define $V(N ; \chi)_{K}:=V(N ; \chi) \cap S(3 / 2, N$, $\chi)_{K}$. See $[U 1, \S 0, \S 1]$ for the details.

\section{$\S 1$. Representations of $S L_{2}$ over finite fields and non-vanishing of Fourier coefficients}

We begin with a summary of representations of $\mathbf{G}:=S L_{2}\left(\mathbf{F}_{p}\right)$ ( $p$ is an odd prime number).

Define subgroups of $\mathbf{G}$ by:

$$
\mathbf{B}:=\left\{\left(\begin{array}{cc}
a & b \\
0 & a^{-1}
\end{array}\right) \mid a \in \mathbf{F}_{p}^{\times}, b \in \mathbf{F}_{p}\right\}, \quad \mathbf{U}:=\left\{\left(\begin{array}{ll}
1 & b \\
0 & 1
\end{array}\right) \mid b \in \mathbf{F}_{p}\right\}
$$

and put

$$
\begin{aligned}
& \psi: \mathbf{F}_{p} \ni u \bmod p \longmapsto \mathbf{e}(u / p):=\exp (2 \pi \sqrt{-1} u / p) \in \mathbf{C}^{\times} \\
& \psi_{a}\left(\left(\begin{array}{ll}
1 & u \\
0 & 1
\end{array}\right)\right):=\psi(a u) \quad\left(a, u \in \mathbf{F}_{p}\right) .
\end{aligned}
$$


The set of all irreducible representations of $\mathbf{U}$ is given by $\left\{\psi_{a} \mid a \in \mathbf{F}_{p}\right\}$. For any Dirichlet character $\chi$ modulo $p$, we define the representations $\underline{\underline{\chi}}$ of $\mathbf{B}$ of degree one by: $\underline{\underline{\chi}}: \mathbf{B} \ni\left(\begin{array}{cc}a & b \\ 0 & a^{-1}\end{array}\right) \mapsto \chi(a) \in \mathbf{C}^{\times}$.

The following facts are well-known.

Proposition 1. ([S, Chapter 7, pp. 54-60]) (1) If $\chi^{2} \neq \mathbf{1}$, Ind $\mathbf{B} \underline{\underline{\mathbf{Z}}} \underline{\underline{\mathrm{f}}}$ is an irreducible representation.

(2) If $\chi=\mathbf{1}$ (the trivial representation), Ind $\mathbf{G}_{\mathbf{B}}^{\mathbf{G}} \mathbf{1}=\mathbf{1} \oplus \mathfrak{C}_{p}$, where $\mathfrak{C}_{p}$ is an irreducible representation of $\mathbf{G}$ of degree $p$ which is called Steinberg representation and $\operatorname{Res}_{\mathbf{U}} \mathfrak{C}_{p} \cong \oplus_{a \in \mathbf{F}_{p}} \psi_{a}$.

(3) If $\chi=(\bar{p})$ (Legendre symbol), Ind $\mathbf{B} \underline{\underline{\mathbf{X}}}=\mathfrak{C}_{(p+1) / 2} \oplus \mathfrak{C}_{(p+1) / 2}^{\prime}$, where $\mathfrak{C}_{(p+1) / 2}$ and $\mathfrak{C}_{(p+1) / 2}^{\prime}$ denote irreducible representations of $\mathbf{G}$ of degree $(p+$ 1) $/ 2$, which are not equivalent to each other and satisfy the following:

$$
\begin{gathered}
\operatorname{Res} \mathbf{U}\left(\mathfrak{C}_{(p+1) / 2}\right) \cong \psi_{0} \oplus\left(\bigoplus_{a \in \mathbf{F}_{p}^{\times 2}} \psi_{a}\right), \\
\operatorname{Res} \mathbf{U}\left(\mathfrak{C}_{(p+1) / 2}^{\prime}\right) \cong \psi_{0} \oplus\left(\bigoplus_{a \in \mathbf{F}_{p}^{\times}-\mathbf{F}_{p}^{\times 2}} \psi_{a}\right) .
\end{gathered}
$$

We call $\mathfrak{C}_{(p+1) / 2}\left(\right.$ resp. $\left.\mathfrak{C}_{(p+1) / 2}^{\prime}\right)$ the residual (resp. non-residual) representation.

Assumption. From now on and until the end of the paper, we assume the following:

$$
\chi^{2}=1
$$

We will determine the explicit $\mathbf{C}$-basis of the irreducible components $\mathbf{1}, \mathfrak{C}_{p}, \mathfrak{C}_{(p+1) / 2}$, and $\mathfrak{C}_{(p+1) / 2}^{\prime}$ in $\operatorname{Ind}_{\mathbf{B}}^{\mathbf{G}} \underline{\underline{\chi}}(\chi=\mathbf{1},(\bar{p}))$.

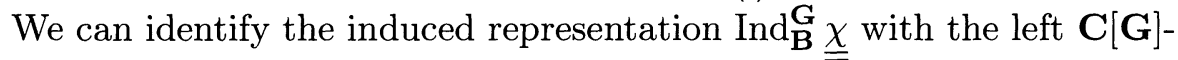

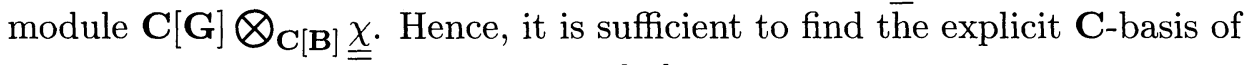
irreducible components of this left $\mathbf{C}[\mathbf{G}]$-module.

Let $e:=\left(\begin{array}{ll}1 & 0 \\ 0 & 1\end{array}\right)$ be the unit element of $\mathbf{G}$ and we choose a basis of the representation space of $\underline{\underline{\chi}}$ by $\varepsilon$, i.e., $\underline{\underline{\chi}}\left(\left(\begin{array}{cc}a & b \\ 0 & a^{-1}\end{array}\right)\right) \varepsilon=\chi(a) \varepsilon$. A system 
of all representatives for $\mathbf{G} / \mathbf{B}$ is given by the $p+1$ elements: $e=\left(\begin{array}{ll}1 & 0 \\ 0 & 1\end{array}\right)$ and $\xi_{a}:=\left(\begin{array}{ll}1 & a \\ 0 & 1\end{array}\right)\left(\begin{array}{cc}0 & 1 \\ -1 & 0\end{array}\right)\left(a \in \mathbf{F}_{p}\right)$. Under these notation, a $\mathbf{C}$-basis of $\operatorname{Ind}_{\mathbf{B}}^{\mathbf{G}} \underline{\underline{\chi}}=\mathbf{C}[\mathbf{G}] \otimes_{\mathbf{C}[\mathbf{B}]} \underline{\underline{\chi}}$ is given by the $p+1$ elements: $e \otimes \varepsilon$ and $\xi_{a} \otimes \varepsilon$ $\left(a \in \overline{\overline{\mathbf{F}}}_{p}\right)$.

Take any element $u \in \mathbf{F}_{p}$ and $h:=\alpha(e \otimes \varepsilon)+\sum_{x \in \mathbf{F}_{p}} \beta_{x}\left(\xi_{x} \otimes \varepsilon\right) \in \operatorname{Ind}_{\mathbf{B}}^{\mathbf{G}} \underline{\underline{\chi}}$ $\left(\alpha, \beta_{x} \in \mathbf{C}\left(x \in \mathbf{F}_{p}\right)\right)$. We have the identity $\left(\begin{array}{ll}1 & u \\ 0 & 1\end{array}\right) \cdot h=\alpha(e \otimes \varepsilon)+$ $\sum_{x \in \mathbf{F}_{p}} \beta_{x}\left(\xi_{u+x} \otimes \varepsilon\right)$. Hence any element $h$ belonging to the representation $\psi_{a}\left(a \in \mathbf{F}_{p}\right)$ is expressed as follows:

$$
\left\{\begin{array}{lll}
h=\alpha(e \otimes \varepsilon)+\beta\left(\sum_{x \in \mathbf{F}_{p}} \xi_{x} \otimes \varepsilon\right) & (\alpha, \beta \in \mathbf{C}) & \text { if } a=0, \\
h=\beta \sum_{x \in \mathbf{F}_{p}} \mathbf{e}(-a x / p)\left(\xi_{x} \otimes \varepsilon\right) & (\beta \in \mathbf{C}) & \text { if } a \neq 0 .
\end{array}\right.
$$

We therefore see that for any non-zero $a, \mathbf{C}\left(\sum_{x \in \mathbf{F}_{p}} \mathbf{e}(-a x / p)\left(\xi_{x} \otimes \varepsilon\right)\right)$ is the $\psi_{a}$-component in the $\mathbf{C}[\mathbf{U}]$-modules $\operatorname{Res} \mathbf{U} \mathfrak{C}_{p}$, $\operatorname{Res} \mathbf{U} \mathfrak{C}_{(p+1) / 2}$, and $\operatorname{Resu} \mathfrak{C}_{(p+1) / 2}^{\prime}$.

We must determine an explicit basis of each $\psi_{0}$-component in the above three $\mathbf{C}[\mathbf{U}]$-modules.

Put for any $a \in \mathbf{F}_{p}, h_{a}:=\sum_{x \in \mathbf{F}_{p}} \mathbf{e}(-a x / p)\left(\xi_{x} \otimes \varepsilon\right) \in \operatorname{Ind}_{\mathbf{B}}^{\mathbf{G}} \underline{\underline{\chi}}$ and put $\mathcal{X}:=\sum_{x \in \mathbf{F}_{p}} \xi_{x} \in \mathbf{C}[\mathbf{G}]$.

We will calculate the element $\mathcal{X} h_{a}=\sum_{x, y \in \mathbf{F}_{p}} \mathbf{e}(-a y / p)\left(\xi_{x} \xi_{y} \otimes \varepsilon\right)$. Since $\xi_{x} \xi_{0}=\left(\begin{array}{cc}-1 & -x \\ 0 & -1\end{array}\right) \in \mathbf{B}$, the part of $y=0$ in the above sum is

$$
\sum_{x \in \mathbf{F}_{p}} \xi_{x} \xi_{0} \otimes \varepsilon=\sum_{x \in \mathbf{F}_{p}} e \otimes \underline{\underline{\chi}}\left(\left(\begin{array}{cc}
-1 & -x \\
0 & -1
\end{array}\right)\right) \varepsilon=\chi(-1) p(e \otimes \varepsilon) .
$$

Next assume that $y \neq 0$. Then

$$
\xi_{x} \xi_{y}=\left(\begin{array}{cc}
x y-1 & -x \\
y & -1
\end{array}\right)=\xi_{x-y^{-1}}\left(\begin{array}{cc}
-y & 0 \\
0 & -y^{-1}
\end{array}\right)\left(\begin{array}{cc}
1 & -y^{-1} \\
0 & 1
\end{array}\right)
$$

Hence

$$
\sum_{\substack{x \in \mathbf{F}_{p} \\ y \in \mathbf{F}_{p}^{\times}}} \mathbf{e}(-a y / p)\left(\xi_{x} \xi_{y} \otimes \varepsilon\right)
$$




$$
\begin{aligned}
& =\sum_{\substack{x \in \mathbf{F}_{p} \\
y \in \mathbf{F}_{p}^{\times}}} \mathbf{e}(-a y / p) \xi_{x-y^{-1}} \otimes \underline{\underline{\chi}}\left(\left(\begin{array}{cc}
-y & 0 \\
0 & -y^{-1}
\end{array}\right)\left(\begin{array}{cc}
1 & -y^{-1} \\
0 & 1
\end{array}\right)\right) \varepsilon \\
& =\sum_{y \in \mathbf{F}_{p}^{\times}} \mathbf{e}(-a y / p) \chi(-y)\left(\sum_{x \in \mathbf{F}_{p}} \xi_{x-y^{-1}} \otimes \varepsilon\right) \\
& =\left(\sum_{y \in \mathbf{F}_{p}^{\times}} \mathbf{e}(a y / p) \chi(y)\right) h_{0} .
\end{aligned}
$$

From these results,

$$
\mathcal{X} h_{a}= \begin{cases}p(e \otimes \varepsilon)+(p-1) h_{0}, & \text { if } \chi=\mathbf{1} \text { and } a=0, \\ p(e \otimes \varepsilon)-h_{0}, & \text { if } \chi=\mathbf{1} \text { and } a \neq 0, \\ \left(\frac{-1}{p}\right) p(e \otimes \varepsilon), & \text { if } \chi=\left(\frac{-}{p}\right) \text { and } a=0, \\ \left(\frac{-1}{p}\right) p(e \otimes \varepsilon)+\left(\frac{a}{p}\right) \mathfrak{g}_{p} h_{0}, & \text { if } \chi=\left(\frac{-}{p}\right) \text { and } a \neq 0,\end{cases}
$$

where $\mathfrak{g}_{p}:=\sum_{x \in \mathbf{F}_{p}^{\times}} \mathbf{e}(x / p)\left(\frac{x}{p}\right)$ is the gauss sum.

Suppose $\chi=\left(\frac{\bar{p}}{)}\right.$ and take a quadratic residue $a \in \mathbf{F}_{p}^{\times}$. Since $h_{a} \in$ $\mathfrak{C}_{(p+1) / 2}, \mathcal{X} h_{a}=\left(\frac{-1}{p}\right) p(e \otimes \varepsilon)+\left(\frac{a}{p}\right) \mathfrak{g}_{p} h_{0}=\mathfrak{g}_{p}\left(\mathfrak{g}_{p}(e \otimes \varepsilon)+h_{0}\right) \in \mathfrak{C}_{(p+1) / 2}$. From the formula $(1.2), \mathbf{C}\left(\mathfrak{g}_{p}(e \otimes \varepsilon)+h_{0}\right) \cong \psi_{0}$ and hence this element gives a basis of the $\psi_{0}$-component of $\mathfrak{C}_{(p+1) / 2}$.

We can find an explicit basis of the $\psi_{0}$-component of $\mathfrak{C}_{p}, \mathfrak{C}_{(p+1) / 2}^{\prime}$ in the same way. The case of $\mathbf{1}$ is trivial. Thus we obtain the following.

Proposition 2. Under the above notation, we have the following explicit expression of irreducible $\mathbf{C}[\mathbf{G}]$-modules in $\operatorname{Ind}_{\mathbf{B}}^{\mathbf{G}} \underline{\underline{\chi}}$.

$$
\begin{array}{ll}
\mathbf{1} & =\mathbf{C}\left((e \otimes \varepsilon)+h_{0}\right), \\
\mathfrak{C}_{p} & =\mathbf{C}\left(p(e \otimes \varepsilon)-h_{0}\right) \oplus\left(\bigoplus_{a \in \mathbf{F}_{p}^{\times}} \mathbf{C} h_{a}\right), \\
\mathfrak{C}_{(p+1) / 2} & =\mathbf{C}\left(\mathfrak{g}_{p}(e \otimes \varepsilon)+h_{0}\right) \oplus\left(\bigoplus_{a \in \mathbf{F}_{p}^{\times 2}} \mathbf{C} h_{a}\right), \\
\mathfrak{C}_{(p+1) / 2}^{\prime} & =\mathbf{C}\left(\mathfrak{g}_{p}(e \otimes \varepsilon)-h_{0}\right) \oplus\left(\bigoplus_{a \in \mathbf{F}_{p}^{\times}-\mathbf{F}_{p}^{\times 2}} \mathbf{C} h_{a}\right) .
\end{array}
$$


Here, each direct summand of the right-hand sides is an irreducible $\mathbf{C}[\mathbf{U}]$ module. Precisely speaking, each first direct summand is isomorphic to $\psi_{0}$ and the other direct summands $\mathbf{C} h_{a}(a \neq 0)$ are isomorphic to $\psi_{a}$ respectively, as $\mathbf{C}[\mathbf{U}]$-module.

From now, we study representations of finite groups which are constructed by modular forms of half-integral weight.

We use following notation until the end of this section.

Let $k$ be a positive integer and $N=4 \times M, M$ is a positive odd integer.

Put $\nu_{p}=\operatorname{ord}_{p}(N)=\operatorname{ord}_{p}(M)$ for any odd prime $p$. We decompose $M=M_{1} M_{2+}, M_{1}:=\prod_{p \mid M, \nu_{p}=1} p$ and $M_{2+}:=\prod_{p \mid M, \nu_{p} \geq 2} p^{\nu_{p}}$.

Let $\chi$ be an even Dirichlet character with $\chi^{2}=\mathbf{1}$ and for any prime $p$, $\chi_{p}$ the $p$-primary component of $\chi$. Moreover we denote the $M_{1}\left(\operatorname{resp} .2 M_{2+}\right)$ component of $\chi$ by $\chi_{1}:=\prod_{p \mid M_{1}} \chi_{p}$ (resp. $\left.\tilde{\chi}:=\prod_{p \mid 2 M_{2+}} \chi_{p}\right)$.

For any positive integer $\alpha \in \mathbf{Z}_{+}$, put

$$
\begin{aligned}
& \mathbf{G}(\alpha):=S L_{2}(\mathbf{Z} / \alpha \mathbf{Z}), \\
& \mathbf{B}(\alpha):=\left\{\left(\begin{array}{l}
* * \\
0
\end{array}\right) \in \mathbf{G}(\alpha)\right\}, \\
& \mathbf{U}(\alpha):=\left\{\left(\begin{array}{ll}
1 & * \\
0 & 1
\end{array}\right) \in \mathbf{G}(\alpha)\right\} .
\end{aligned}
$$

Moreover if $(\alpha, \beta)=1$, we naturally identify as follows: $\mathbf{G}(\alpha \beta)=\mathbf{G}(\alpha) \times$ $\mathbf{G}(\beta)$ and for simplicity, we shortly write $\mathbf{G}_{1}:=\mathbf{G}\left(M_{1}\right), \mathbf{B}_{1}:=\mathbf{B}\left(M_{1}\right)$, and $\tilde{\mathbf{B}}:=\mathbf{B}\left(4 M_{2+}\right)$.

For any positive integer $\alpha$ divisible by 4 , put $\Delta(\alpha):=\left\{\gamma^{*} \mid \gamma \in \Gamma(\alpha)\right\}$, $\Delta_{1}(\alpha):=\left\{\gamma^{*} \mid \gamma \in \Gamma_{1}(\alpha)\right\}$, and $\Delta_{0}(\alpha):=\left\{\gamma^{*} \mid \gamma \in \Gamma_{0}(\alpha)\right\}$ (cf. $\S 0(\mathrm{c})$ ).

Under these notation, we have that $\Delta_{0}\left(4 M_{2+}\right) \triangleright \Delta(N)=\Delta\left(4 M_{1} M_{2+}\right)$ and that

$$
\Delta_{0}\left(4 M_{2+}\right) / \Delta(N) \cong \Gamma_{0}\left(4 M_{2+}\right) / \Gamma(N) \cong \tilde{\mathbf{B}} \times \mathbf{G}_{1}
$$

From this we can define the representation $\boldsymbol{\pi}$ of the group $\tilde{\mathbf{B}} \times \mathbf{G}_{1}$ on $S(k+1 / 2, \Delta(N))$ as follows:

$$
[\boldsymbol{\pi}(\gamma \bmod (N))] f:=f \mid \gamma^{*-1}, \quad(f \in S(k+1 / 2, \Delta(N))),
$$

where $\gamma \bmod (N) \in \Gamma_{0}\left(4 M_{2+}\right) / \Gamma(N) \simeq \tilde{\mathbf{B}} \times \mathbf{G}_{1}$. We consider $S(k+$ $1 / 2, \Delta(N))$ as a left $\mathbf{C}\left[\tilde{\mathbf{B}} \times \mathbf{G}_{1}\right]$-module by the above representation $\boldsymbol{\pi}$. 
Let $\boldsymbol{\pi}_{f}$ be the left $\mathbf{C}\left[\tilde{\mathbf{B}} \times \mathbf{G}_{1}\right]$-module generated by a non-zero cusp form $f \in S(k+1 / 2, N, \chi)$, i.e.,

$$
\boldsymbol{\pi}_{f}:=\mathbf{C}\left[\tilde{\mathbf{B}} \times \mathbf{G}_{1}\right] f=\left\langle f \mid \gamma^{*} ; \gamma \in \Gamma_{0}\left(4 M_{2+}\right)\right\rangle_{\mathbf{C}} .
$$

Moreover we can define the following one-dimensional representations $\underline{\underline{\chi}}$, $\underline{\underline{\chi_{1}}}, \underline{\underline{\tilde{\chi}}}$ and $\underline{\underline{\chi_{p}}}$ :

$$
\begin{aligned}
& \underline{\underline{\chi}}: \mathbf{B}(N) \ni\left(\begin{array}{cc}
a & b \\
0 & a^{-1}
\end{array}\right) \longmapsto \chi(a) \in \mathbf{C}^{\times}, \\
& \underline{\underline{\chi_{1}}}: \mathbf{B}_{1} \quad \ni\left(\begin{array}{cc}
a & b \\
0 & a^{-1}
\end{array}\right) \longmapsto \chi_{1}(a) \in \mathbf{C}^{\times} \\
& \underline{\underline{\tilde{\chi}}}: \tilde{\mathbf{B}} \quad \ni\left(\begin{array}{cc}
a & b \\
0 & a^{-1}
\end{array}\right) \longmapsto \tilde{\chi}(a) \in \mathbf{C}^{\times}, \\
& \underline{\underline{\chi_{p}}}: \mathbf{B}\left(p^{\nu_{p}}\right) \ni\left(\begin{array}{cc}
a & b \\
0 & a^{-1}
\end{array}\right) \longmapsto \chi_{p}(a) \in \mathbf{C}^{\times} \text {. }
\end{aligned}
$$

Then we have a canonical identification $\underline{\underline{\chi}}=\underline{\underline{\tilde{\chi}}} \otimes \underline{\underline{\chi_{1}}}=\underline{\underline{\tilde{\chi}}} \otimes\left(\otimes_{p \mid M_{1}} \underline{\underline{\chi_{p}}}\right)$.

For any $f \in S(k+1 / 2, N, \chi)$ and any $\gamma=\left(\begin{array}{ll}a & b \\ c & d\end{array}\right) \in \Gamma_{0}(N), \boldsymbol{\pi}(\gamma \bmod$ $(N)) f=f \mid\left(\begin{array}{cc}d & -b \\ -c & a\end{array}\right)^{*}=\chi(a) f$. Hence we get the isomorphism $\underline{\underline{\chi}} \cong \mathbf{C} f$ as $\mathbf{B}(N)$-modules. We denote the basis of the representation space of $\underline{\underline{\chi}}$ by $\varepsilon$. Then we get the following proposition by easy computation.

Proposition 3. For any non-zero $f \in S(k+1 / 2, N, \chi)$, the following map gives an surjective homomorphism of $\tilde{\mathbf{B}} \times \mathbf{G}_{1}$-modules:

$$
\Phi=\Phi_{f}: \operatorname{Ind}_{\tilde{\mathbf{B}} \times \mathbf{B}_{1}}^{\tilde{\mathbf{B}} \times \mathbf{G}_{1}} \underline{\underline{\chi}} \longrightarrow \boldsymbol{\pi}_{f}, \quad\left(\sum_{\eta} a_{\eta} \eta \otimes \varepsilon \longmapsto \sum_{\eta} a_{\eta} \boldsymbol{\pi}(\eta) f\right),
$$

where $a_{\eta} \in \mathbf{C}$ and $\eta$ is taken over all representatives for $\left(\tilde{\mathbf{B}} \times \mathbf{G}_{1}\right) /\left(\tilde{\mathbf{B}} \times \mathbf{B}_{1}\right)$. We can, therefore, identify $\boldsymbol{\pi}_{f}$ with a subrepresentation of $\operatorname{Ind}_{\tilde{\mathbf{B}} \times \mathbf{B}_{1}}^{\tilde{\tilde{\mathbf{B}}} \times \mathbf{G}_{1}}$.

We have canonical identity:

$$
\operatorname{Ind}_{\tilde{\mathbf{B}} \times \mathbf{B}_{1}}^{\tilde{\mathbf{B}} \times \mathbf{G}_{1}} \underline{\underline{\chi}}=\underline{\underline{\tilde{\chi}}} \otimes \operatorname{Ind}_{\mathbf{B}_{1}}^{\mathbf{G}_{1}} \underline{\underline{\chi_{1}}}=\underline{\underline{\tilde{\chi}}} \otimes\left(\bigotimes_{p \mid M_{1}} \operatorname{Ind}_{\mathbf{B}(p)}^{\mathbf{G}(p)} \underline{\underline{\underline{\chi_{p}}}}\right) .
$$


From Proposition 1, we know that each $\operatorname{Ind}_{\mathbf{B}(p)}^{\mathbf{G}(p)} \underline{\underline{\chi_{p}}}$ is the direct sum of two distinct irreducible submodules. We therefore have the following decomposition

$$
\operatorname{Ind}_{\tilde{\mathbf{B}} \times \mathbf{B}_{1}}^{\tilde{\mathbf{B}} \times \mathbf{G}_{1}} \underline{\underline{\chi}}=\bigoplus_{\left(\theta_{p}\right)_{p \mid M_{1}}}\left(\underline{\underline{\tilde{\chi}}} \otimes\left(\bigotimes_{p \mid M_{1}} \theta_{p}\right)\right)
$$

where $\theta_{p}$ is taken over the set of irreducible $\mathbf{C}[\mathbf{G}(p)]$-modules $\left\{\mathbf{1}_{\mathbf{G}(p)}, \mathfrak{C}_{p}\right\}$ or $\left\{\mathfrak{C}_{(p+1) / 2}, \mathfrak{C}_{(p+1) / 2}^{\prime}\right\}$ accordingly to $\chi_{p}=\mathbf{1}_{\mathbf{G}(p)}$ or $(\bar{p})$ (cf. Proposition 1 ). We note that $\underline{\tilde{\chi}} \otimes\left(\bigotimes_{p \mid M_{1}} \theta_{p}\right)$ are irreducible $\mathbf{C}[\mathbf{G}]$-modules and not equivalent to each other.

Now we more closely study the representation $\pi_{f}$ by using Proposition 2. We need some notational preliminaries.

For any prime divisor $p$ of $M_{1}$, let $\varepsilon_{p}$ be a basis of one-dimensional representation space of $\underline{\underline{\chi_{p}}}$. Put $e_{p}:=\left(\begin{array}{ll}1 & 0 \\ 0 & 1\end{array}\right) \in \mathbf{G}(p)$ and $\xi_{p}(a):=\left(\begin{array}{ll}1 & a \\ 0 & 1\end{array}\right)\left(\begin{array}{cc}0 & 1 \\ -1 & 0\end{array}\right) \in$ $\mathbf{G}(p)$ for $a \in \mathbf{F}_{p}$ and $\mathcal{X}_{p}(a):=\sum_{x \in \mathbf{F}_{p}} \mathbf{e}(-a x / p) \xi_{p}(x) \in \mathbf{C}[\mathbf{G}(p)]$. Moreover let $\tilde{\varepsilon}$ be a basis of $\underline{\underline{\chi}}$.

For any prime divisor $p$ of $M_{1}$ and any $x \in \mathbf{F}_{p}$, we choose an element $\gamma_{p}(x) \in S L_{2}(\mathbf{Z})$ such that

$$
S L_{2}(\mathbf{Z}) \ni \gamma_{p}(x) \equiv \begin{cases}\left(\begin{array}{ll}
1 & 0 \\
0 & 1
\end{array}\right) & \bmod N / p \\
\xi_{p}(x) & \bmod p\end{cases}
$$

and define an operator $X_{p}\left(p \mid M_{1}\right)$ as follows:

$$
f \mid X_{p}:=\sum_{x \in \mathbf{F}_{p}} \boldsymbol{\pi}\left(\gamma_{p}(x) \bmod (N)\right) f, \quad f \in S(k+1 / 2, N, \chi) .
$$

In [U1, 11$]$, we defined several fundamental operators on $S(k+1 / 2, N, \chi)$ : $U(p), \widetilde{W}(p)$, and $Y_{p}$. The above operator $X_{p}$ can be express by those.

Proposition 4. Let $p$ be any prime divisor of $M_{1}$ and $f$ any element of $S(k+1 / 2, N, \chi)$. Then the following hold.

(1) $f\left|X_{p}=p^{-k / 2+3 / 4} f\right| \widetilde{W}(p) U(p)$. Hence the map $f \mapsto f \mid X_{p}$ gives an operator on $S(k+1 / 2, N, \chi)$.

(2) $f\left|Y_{p} U(p)=f\right| U(p) X_{p}$. 
(3) $f \mid X_{p}^{2}= \begin{cases}(p-1) f \mid X_{p}+p f, & \text { if } \chi_{p}=\mathbf{1}, \\ \left(\frac{-1}{p}\right) p f, & \text { if } \chi_{p}=\left(\frac{-}{p}\right) .\end{cases}$

Hence $X_{p}$ is a semi-simple operator on $S(k+1 / 2, N, \chi)$.

Proof. (1) We keep to the notation in [U1, §1]. We have from the definition [U1, p. 151] $\widetilde{W}(p)=\gamma_{p}^{*} \tilde{\delta}_{p}$, where $\gamma_{p} \in S L_{2}(\mathbf{Z})$ is satisfied the following condition:

$$
\gamma_{p} \equiv \begin{cases}\left(\begin{array}{cc}
0 & -1 \\
1 & 0
\end{array}\right) & (\bmod p) \\
\left(\begin{array}{ll}
1 & 0 \\
0 & 1
\end{array}\right) & (\bmod N / p)\end{cases}
$$

and $\tilde{\delta}_{p}=\left(\left(\begin{array}{ll}p & 0 \\ 0 & 1\end{array}\right), p^{-k / 2-1 / 4}\right)$. From $[\mathrm{U} 1,(1.19)]$,

$$
\begin{aligned}
f \mid \widetilde{W}(p) U(p) & =p^{k / 2-3 / 4} \sum_{a \in \mathbf{F}_{p}} f \mid \widetilde{W}(p)\left(\left(\begin{array}{ll}
1 & 0 \\
0 & p
\end{array}\right), p^{k / 2+1 / 4}\right)\left(\begin{array}{ll}
1 & a \\
0 & 1
\end{array}\right)^{*} \\
& =p^{k / 2-3 / 4} \sum_{a \in \mathbf{F}_{p}} f \mid \gamma_{p}^{*}\left(\left(\begin{array}{ll}
p & 0 \\
0 & p
\end{array}\right), 1\right)\left(\begin{array}{ll}
1 & a \\
0 & 1
\end{array}\right)^{*} \\
& =p^{k / 2-3 / 4} \sum_{a \in \mathbf{F}_{p}} f \mid \gamma_{p}^{*}\left(\begin{array}{ll}
1 & a \\
0 & 1
\end{array}\right)^{*}
\end{aligned}
$$

By using the notation $\gamma_{p}(a)$ (cf. (1.7)), we can easily calculate for any $a \in \mathbf{F}_{p}, f \mid \gamma_{p}^{*}\left(\begin{array}{ll}1 & a \\ 0 & 1\end{array}\right)^{*}=\boldsymbol{\pi}\left(\gamma_{p}(-a) \bmod (N)\right) f$. We therefore have

$$
\begin{aligned}
f \mid \widetilde{W}(p) U(p) & =p^{k / 2-3 / 4} \sum_{a \in \mathbf{F}_{p}} f \mid \gamma_{p}^{*}\left(\begin{array}{ll}
1 & a \\
0 & 1
\end{array}\right)^{*} \\
& =p^{k / 2-3 / 4} \sum_{a \in \mathbf{F}_{p}} \boldsymbol{\pi}\left(\gamma_{p}(-a) \bmod (N)\right) f=p^{k / 2-3 / 4} f \mid X_{p} .
\end{aligned}
$$

From this and [U1, (1.22)], $f \mapsto f \mid X_{p}$ gives an operator on $S(k+1 / 2, N, \chi)$.

(2) From the definition of $Y_{p}$ (cf. [U1, p. 155]) and the above (1),

$$
\begin{aligned}
p^{k / 2-3 / 4} f \mid Y_{p} U(p) & =f \mid U(p) \widetilde{W}(p) U(p) \\
& =(f \mid U(p))\left|\widetilde{W}(p) U(p)=p^{k / 2-3 / 4} f\right| U(p) X_{p} .
\end{aligned}
$$


(3) The map $f \mapsto f \mid U(p)$ gives an isomorphism from $S(k+1 / 2, N, \chi)$ onto $S(k+1 / 2, N, \chi(\underline{p}))$ (cf. [U1, (1.28)]). Hence it follows from the above (2) that $U(p)^{-1} Y_{p} U(p)=X_{p}$ on $S(k+1 / 2, N, \chi)$. By using this relation, we can deduce properties of $X_{p}$ from those of $Y_{p}$. In particular, from [U1, Proposition (1.27)], we have the above relation (3).

Take any non-zero $f \in S(k+1 / 2, N, \chi)$ and assume that $\boldsymbol{\pi}_{f}$ is irreducible. From Proposition 3 and (1.6), there exists a system of irreducible representations $\left(\theta_{p}\right)_{p \mid M_{1}}$ such that $\Phi_{f}: \underline{\tilde{\chi}} \otimes\left(\otimes_{p \mid M_{1}} \theta_{p}\right) \cong \boldsymbol{\pi}_{f}$. Moreover for any prime divisor $q$ of $M_{1}$,

$$
\operatorname{Ker}\left(\Phi_{f}\right) \supseteq \underline{\underline{\tilde{\chi}}} \otimes\left(\bigotimes_{p \mid M_{1}, p \neq q} \operatorname{Ind}_{\mathbf{B}(p)}^{\mathbf{G}(p)} \underline{\underline{\chi_{p}}}\right) \otimes \theta_{q}^{\prime}
$$

where $\theta_{q}^{\prime}$ is the irreducible component such that $\operatorname{Ind}_{\mathbf{B}(q)}^{\mathbf{G}(q)} \underline{\underline{\chi_{q}}}=\theta_{q} \oplus \theta_{q}^{\prime}$.

Set the elements $\alpha_{p}, \alpha_{p}^{\prime} \in \mathbf{C}[\mathbf{G}(p)]$ such that

$$
\left(\alpha_{p}, \alpha_{p}^{\prime}\right):= \begin{cases}\left(e_{p}+\mathcal{X}_{p}(0), p e_{p}-\mathcal{X}_{p}(0)\right), & \text { if } \theta_{p}=\mathbf{1}_{\mathbf{G}(p)}, \\ \left(p e_{p}-\mathcal{X}_{p}(0), e_{p}+\mathcal{X}_{p}(0)\right), & \text { if } \theta_{p}=\mathfrak{C}_{p}, \\ \left(\mathfrak{g}_{p} e_{p}+\mathcal{X}_{p}(0), \mathfrak{g}_{p} e_{p}-\mathcal{X}_{p}(0)\right), & \text { if } \theta_{p}=\mathfrak{C}_{(p+1) / 2}, \\ \left(\mathfrak{g}_{p} e_{p}-\mathcal{X}_{p}(0), \mathfrak{g}_{p} e_{p}+\mathcal{X}_{p}(0)\right), & \text { if } \theta_{p}=\mathfrak{C}_{(p+1) / 2}^{\prime}\end{cases}
$$

Then $\alpha_{p} \otimes \varepsilon_{p} \in \theta_{p}, \alpha_{p}^{\prime} \otimes \varepsilon_{p} \in \theta_{p}^{\prime}$ and

$$
\tilde{\varepsilon} \otimes\left(\bigotimes_{p \mid M_{1}, p \neq q} e_{p} \otimes \varepsilon_{p}\right) \otimes\left(\alpha_{q}^{\prime} \otimes \varepsilon_{q}\right) \in \operatorname{Ker}\left(\Phi_{f}\right) .
$$

We therefore have

$$
\begin{aligned}
0 & =\Phi_{f}\left(\tilde{\varepsilon} \otimes\left(\bigotimes_{p \mid M_{1}, p \neq q} e_{p} \otimes \varepsilon_{p}\right) \otimes\left(\alpha_{q}^{\prime} \otimes \varepsilon_{q}\right)\right) \\
& = \begin{cases}q f-f \mid X_{q}, & \text { if } \theta_{q}=\mathbf{1}_{\mathbf{G}(q)}, \\
f+f \mid X_{q}, & \text { if } \theta_{q}=\mathfrak{C}_{q}, \\
\mathfrak{g}_{q} f-f \mid X_{q}, & \text { if } \theta_{q}=\mathfrak{C}_{(q+1) / 2}, \\
\mathfrak{g}_{q} f+f \mid X_{q}, & \text { if } \theta_{q}=\mathfrak{C}_{(q+1) / 2}^{\prime} .\end{cases}
\end{aligned}
$$

In other wards, $f$ is a common eigenform of $X_{p}$ for all prime divisors $p \mid M_{1}$. 
Conversely, assume that $f$ is a common eigenform of $X_{p}, p \mid M_{1}$.

Decompose $\boldsymbol{\pi}_{f}$ into irreducible components: $\boldsymbol{\pi}_{f}=\rho_{1} \oplus \cdots \oplus \rho_{m}$ and let $f=f_{1}+\cdots+f_{m}, f_{i} \in \rho_{i}(i=1, \ldots, m)$.

We apply the following operator to the above: $\mathcal{M}:=\left|\tilde{\mathbf{B}} \times \mathbf{B}_{1}\right|^{-1} \sum_{\gamma}$ $\bar{\chi}(d) \gamma^{*}$, where $\gamma=\left(\begin{array}{ll}a & b \\ c & d\end{array}\right)$ is taken over all representatives of $\Gamma_{0}(N) / \Gamma(N)$.

We easily see that $f=f \mid \mathcal{M}$ and for any $i, f_{i} \mid \mathcal{M} \in \rho_{i} \cap S(k+$ $1 / 2, N, \chi)$. From this, without loss of generality, we can assume that $f_{i} \in$ $S(k+1 / 2, N, \chi)$ and $f_{i} \neq 0(i=1, \ldots, m)$. Then $\rho_{i}$ is generated by a non-zero form $f_{i}$ for all $i$. It follows from (1.11) that all $f_{i}$ 's are common eigenforms of $X_{p}, p \mid M_{1}$.

Set $f_{i} \mid X_{p}=\sigma_{p}^{(i)} f_{i},\left(i=1, \ldots, m, p \mid M_{1}, \sigma_{p}^{(i)} \in \mathbf{C}\right)$.

Since $\boldsymbol{\pi}_{f}$ is identified with subrepresentation of $\operatorname{Ind}_{\tilde{\mathbf{B}} \times \mathbf{B}_{1}}^{\tilde{\mathbf{B}} \times \mathbf{G}_{1}} \underline{\underline{\chi}}, \rho_{i}(i=$ $1, \ldots, m)$ are not equivalent to each other (cf. (1.6)). Hence, from (1.11), the systems of eigenvalues $\left(\sigma_{p}^{(1)}\right)_{p \mid M_{1}}, \ldots,\left(\sigma_{p}^{(m)}\right)_{p \mid M_{1}}$ are different from each other. Therefore we see that $m=1$ and $\boldsymbol{\pi}_{f}$ is irreducible.

Thus we get the following results.

Proposition 5. Let the notation be the same as above. For $(0 \neq)$ $f \in S(k+1 / 2, N, \chi)$, the following hold good.

(1) $\boldsymbol{\pi}_{f}$ is an irreducible $\mathbf{C}\left[\tilde{\mathbf{B}} \times \mathbf{G}_{1}\right]$-module if and only if $f$ is a common eigenform of $X_{p}, p \mid M_{1}$.

(2) Suppose that $\boldsymbol{\pi}_{f}$ is irreducible. Then $\boldsymbol{\pi}_{f} \cong \underline{\underline{\chi}} \otimes\left(\otimes_{p \mid M_{1}} \theta_{p}\right)$, where $\theta_{p}$ 's are irreducible $\mathbf{C}[\mathbf{G}(p)]$-modules. Let $\sigma_{p}\left(p \mid M_{1}\right)$ be an eigen value of $f$ on $X_{p}: f \mid X_{p}=\sigma_{p} f$. Then $\sigma_{p}$ is given by the following table.

$$
\sigma_{p}= \begin{cases}p, & \text { if } \chi_{p}=\mathbf{1} \quad \text { and } \theta_{p}=\mathbf{1}_{\mathbf{G}(p)}, \\ -1, & \text { if } \chi_{p}=\mathbf{1} \\ \mathfrak{g}_{p}, & \text { if } \chi_{p}=(\bar{p}) \text { and } \theta_{p}=\mathfrak{C}_{p}, \\ -\mathfrak{g}_{p}, & \text { if } \chi_{p}=(\bar{p}) \text { and } \theta_{p}=\mathfrak{C}_{(p+1) / 2}^{\prime},\end{cases}
$$

Now we will study relations between the above representations and Fourier coefficients. 
Let $f$ be a non-zero element of $S(k+1 / 2, N, \chi)$ and take any system $\left(a_{p}\right)_{p \mid M_{1}} \in \prod_{p \mid M_{1}} \mathbf{F}_{p}$. Then

$$
\begin{aligned}
\Phi_{f}\left(\left(\tilde{\varepsilon} \otimes\left(\bigotimes_{p \mid M_{1}} \mathcal{X}_{p}\left(a_{p}\right) \otimes \varepsilon_{p}\right)\right)\right. \\
=\sum_{\substack{\left(x_{p}\right)_{p \mid M_{1}} \\
x_{p} \in \mathbf{F}_{p}}} \prod_{p \mid M_{1}} \mathbf{e}\left(-a_{p} x_{p} / p\right) \boldsymbol{\pi}\left(\gamma\left(\left(x_{p}\right)\right) \bmod (N)\right) f
\end{aligned}
$$

where $\gamma\left(\left(x_{p}\right)\right) \in S L_{2}(\mathbf{Z})$ is an element satisfied the following condition:

$$
\gamma\left(\left(x_{p}\right)\right) \equiv\left\{\begin{array}{l}
\left(\begin{array}{ll}
1 & 0 \\
0 & 1
\end{array}\right) \bmod 4 M_{2+}, \\
\xi_{p}\left(x_{p}\right) \bmod p
\end{array} \quad \text { for all prime divisors } p \text { of } M_{1} .\right.
$$

Choose a matrix $\gamma_{M_{1}} \in S L_{2}(\mathbf{Z})$ and an element $x \in \mathbf{Z}$ for $\left(x_{p}\right)_{p \mid M_{1}} \in$ $\prod_{p \mid M_{1}} \mathbf{F}_{p}$ such that

$$
\gamma_{M_{1}} \equiv\left\{\begin{array} { l } 
{ ( \begin{array} { l l } 
{ 1 } & { 0 } \\
{ 0 } & { 1 }
\end{array} ) \operatorname { m o d } 4 M _ { 2 + } , } \\
{ ( \begin{array} { c c } 
{ 0 } & { - 1 } \\
{ 1 } & { 0 }
\end{array} ) \operatorname { m o d } M _ { 1 } , }
\end{array} \text { and } \quad x \equiv \left\{\begin{array}{l}
0 \bmod 4 M_{2+} \\
x_{p} \bmod p \text { for all } p \mid M_{1}
\end{array}\right.\right.
$$

and put $\alpha\left(\left(x_{p}\right)\right):=\left(\begin{array}{ll}1 & x \\ 0 & 1\end{array}\right)$. Then we note that $\gamma\left(\left(x_{p}\right)\right) \gamma_{M_{1}} \alpha\left(\left(x_{p}\right)\right)^{-1} \in$ $\Gamma(N)$. Moreover set $g:=f \mid \widetilde{W}\left(M_{1}\right)=\sum_{n=1}^{\infty} b(n) \mathbf{e}(n z) \in S(k+1 / 2, N$, $\chi\left(\frac{M_{1}}{)}\right)$. Hence

$$
\begin{aligned}
& \Phi_{f}\left(\tilde{\varepsilon} \otimes \bigotimes_{p \mid M_{1}}\left(\mathcal{X}_{p}\left(a_{p}\right) \otimes \varepsilon_{p}\right)\right) \\
&=\sum_{\substack{\left(x_{p}\right)_{p \mid M_{1}} \\
x_{p} \in \mathbf{F}_{p}}} \prod_{p \mid M_{1}} \mathbf{e}\left(-a_{p} x_{p} / p\right) f \mid \gamma_{M_{1}}^{*} \alpha\left(\left(x_{p}\right)\right)^{*-1} \\
&=\sum_{\substack{\left(x_{p}\right)_{p \mid M_{1}} \\
x_{p} \in \mathbf{F}_{p}}} \prod_{p \mid M_{1}} \mathbf{e}\left(-a_{p} x_{p} / p\right) f \mid \widetilde{W}\left(M_{1}\right) \tilde{\delta}_{M_{1}}^{-1} \alpha\left(\left(x_{p}\right)\right)^{*-1} \\
&=M_{1}-k / 2-1 / 4 \\
& \sum_{\substack{\left(x_{p}\right)_{p \mid M_{1}} \\
x_{p} \in \mathbf{F}_{p}}} \prod_{p \mid M_{1}} \mathbf{e}\left(-a_{p} x_{p} / p\right) g\left((z-x) / M_{1}\right)
\end{aligned}
$$




$$
=M_{1}^{-k / 2-1 / 4} \sum_{n=1}^{\infty} b(n) \mathbf{e}\left(n z / M_{1}\right) \sum_{\substack{\left(x_{p}\right)_{p \mid M_{1}} \\ x_{p} \in \mathbf{F}_{p}}}\left(\prod_{p \mid M_{1}} \mathbf{e}\left(-a_{p} x_{p} / p\right)\right) \mathbf{e}\left(-n x / M_{1}\right)
$$

Since the G.C.D. of all $M_{1} / p\left(p\right.$ : prime divisor of $\left.M_{1}\right)$ is 1 , there exist integers $u_{p} \in \mathbf{Z}$ such that $\sum_{p \mid M_{1}} u_{p} \cdot\left(M_{1} / p\right)=1$. We therefore have

$$
\begin{aligned}
\sum_{\substack{\left(x_{p}\right)_{p \mid M_{1}} \\
x_{p} \in \mathbf{F}_{p}}}\left(\prod_{p \mid M_{1}} \mathbf{e}\left(-a_{p} x_{p} / p\right)\right) \mathbf{e}\left(-n x / M_{1}\right) \\
=\sum_{\substack{\left(x_{p}\right)_{p \mid M_{1}} \\
x_{p} \in \mathbf{F}_{p}}} \prod_{p \mid M_{1}} \mathbf{e}\left(\left(-a_{p} x_{p}-n x u_{p}\right) / p\right) \\
=\prod_{p \mid M_{1} x_{p} \in \mathbf{F}_{p}} \sum \mathbf{e}\left(\left(-a_{p} x_{p}-n x_{p} u_{p}\right) / p\right) \\
= \begin{cases}M_{1}, & \text { if }-a_{p} \equiv n u_{p} \quad(\bmod p) \text { for all } p \mid M_{1}, \\
0, & \text { otherwise. }\end{cases}
\end{aligned}
$$

Here, we use the condition: $x \equiv x_{p} \quad(\bmod p)$.

Since $-a_{p} \equiv n u_{p} \quad(\bmod p) \Leftrightarrow-a_{p}\left(M_{1} / p\right) \equiv n \quad(\bmod p)$, we get the following.

$$
\begin{aligned}
& \Phi_{f}\left(\tilde{\varepsilon} \otimes \bigotimes_{p \mid M_{1}}\left(\mathcal{X}_{p}\left(a_{p}\right) \otimes \varepsilon_{p}\right)\right) \\
&=M_{1}^{-k / 2+3 / 4} \sum_{\substack{n \equiv-a_{p}\left(M_{1} / p\right) \bmod (p) \\
p \mid M_{1}}} b(n) \mathbf{e}\left(n z / M_{1}\right)
\end{aligned}
$$

where $g:=f \mid \widetilde{W}\left(M_{1}\right)=\sum_{n=1}^{\infty} b(n) \mathbf{e}(n z)$.

Moreover we suppose that $\boldsymbol{\pi}_{f}$ is irreducible. In this case, there are irreducible $\mathbf{C}[\mathbf{G}(p)]$-modules $\theta_{p}\left(p \mid M_{1}\right)$ such that $\underline{\tilde{\chi}} \otimes \bigotimes_{p \mid M_{1}} \theta_{p} \cong \boldsymbol{\pi}_{f}$ via $\Phi_{f}$.

We use the notation $\alpha_{p}$ and $\alpha_{p}^{\prime}$ of (1.9). Then in all cases of $\theta_{p}=\mathbf{1}_{\mathbf{G}(p)}$, $\mathfrak{C}_{p}, \mathfrak{C}_{(p+1) / 2}$, or $\mathfrak{C}_{(p+1) / 2}^{\prime}$, we can express that $\mathcal{X}_{p}(0)=c_{0} \times \alpha_{p}+c_{1} \times \alpha_{p}^{\prime}$ with a non-zero constant $c_{0}$ and a constant $c_{1}$. And from the formula (1.10), it follows that for any system $\left(a_{p}\right)_{p \mid M_{1}} \in \prod_{p \mid M_{1}} \mathbf{F}_{p}$, there exists a non-zero 
element $c_{2}$ such that

$$
\begin{aligned}
\tilde{\varepsilon} \otimes \bigotimes_{p \mid M_{1}}\left(\mathcal{X}_{p}\left(a_{p}\right)\right. & \left.\otimes \varepsilon_{p}\right) \\
\equiv c_{2} \tilde{\varepsilon} & \otimes\left(\bigotimes_{p \mid M_{1}, a_{p} \neq 0}\left(\mathcal{X}_{p}\left(a_{p}\right) \otimes \varepsilon_{p}\right)\right) \\
& \otimes\left(\bigotimes_{p \mid M_{1}, a_{p}=0}\left(\alpha_{p} \otimes \varepsilon\right)\right)\left(\bmod \operatorname{Ker} \Phi_{f}\right) .
\end{aligned}
$$

Now we set the following notation for any prime divisor $p$ of $M_{1}$ :

$$
S_{p}^{\times}:=\left\{\begin{array}{ll}
\emptyset, & \text { if } \theta_{p}=\mathbf{1}_{\mathbf{G}(p)}, \\
\mathbf{F}_{p}^{\times}, & \text {if } \theta_{p}=\mathfrak{C}_{p}, \\
\mathbf{F}_{p}^{\times 2}, & \text { if } \theta_{p}=\mathfrak{C}_{(p+1) / 2}, \\
\mathbf{F}_{p}^{\times}-\mathbf{F}_{p}^{\times 2}, & \text { if } \theta_{p}=\mathfrak{C}_{(p+1) / 2}^{\prime},
\end{array}\right\} \text { and } S_{p}:=S_{p}^{\times} \cup\{0\} .
$$

From the formula of Proposition 2, we see that $S_{p}^{\times}=\left\{a \in \mathbf{F}_{p}^{\times} \mid \mathcal{X}_{p}(a) \otimes \varepsilon_{p} \in\right.$ $\left.\theta_{p}\right\}$.

From (1.14), we see that for any system $\left(a_{p}\right)_{p \mid M_{1}} \in \prod_{p \mid M_{1}} \mathbf{F}_{p}$,

$$
\begin{aligned}
& \Phi_{f}\left(\tilde{\varepsilon} \otimes\left(\bigotimes_{p \mid M_{1}} \mathcal{X}_{p}\left(a_{p}\right) \otimes \varepsilon_{p}\right)\right) \neq 0 \\
& \Longleftrightarrow \Phi_{f}\left(\tilde{\varepsilon} \otimes\left(\bigotimes_{p \mid M_{1}, a_{p} \neq 0} \mathcal{X}_{p}\left(a_{p}\right) \otimes \varepsilon_{p}\right) \otimes\left(\bigotimes_{p \mid M_{1}, a_{p}=0} \alpha_{p} \otimes \varepsilon_{p}\right)\right) \neq 0 \\
& \Longleftrightarrow \tilde{\varepsilon} \otimes\left(\bigotimes_{p \mid M_{1}, a_{p} \neq 0} \mathcal{X}_{p}\left(a_{p}\right) \otimes \varepsilon_{p}\right) \otimes\left(\bigotimes_{p \mid M_{1}, a_{p}=0} \alpha_{p} \otimes \varepsilon_{p}\right) \in \underline{\underline{\chi}} \otimes\left(\bigotimes_{p \mid M_{1}} \theta_{p}\right) \\
& \Longleftrightarrow \mathcal{X}_{p}\left(a_{p}\right) \otimes \varepsilon_{p} \in \theta_{p} \text { for any } p \mid M_{1} \text { such that } a_{p} \neq 0 \\
& \Longleftrightarrow a_{p} \in S_{p}^{\times} \text {for any } p \mid M_{1} \text { such that } a_{p} \neq 0 \\
& \Longleftrightarrow\left(a_{p}\right)_{p \mid M_{1}} \in \prod_{p \mid M_{1}} S_{p}
\end{aligned}
$$

Combining the above with the formula (1.13), we obtain the following theorem.

THEOREM 1. Let $f$ be a non-zero element of $S(k+1 / 2, N, \chi)$ and $g:=f \mid \widetilde{W}\left(M_{1}\right)=\sum_{n=1}^{\infty} b(n) \mathbf{e}(n z) \in S\left(k+1 / 2, N, \chi\left(\underline{M_{1}}\right)\right)$. Suppose that 
$\boldsymbol{\pi}_{f}$ is irreducible and $\boldsymbol{\pi}_{f} \cong \underline{\underline{\tilde{\chi}}} \otimes \bigotimes_{p \mid M_{1}} \theta_{p}$ with irreducible $\mathbf{C}[\mathbf{G}(p)]$-modules $\theta_{p}$.

Then for $a\left(a_{p}\right)_{p \mid M_{1}} \in \prod_{p \mid M_{1}} \mathbf{F}_{p}$, we have the following:

$$
\left(a_{p}\right)_{p \mid M_{1}} \in \prod_{p \mid M_{1}} S_{p} \Longleftrightarrow \begin{gathered}
\text { "there exists } n \in \mathbf{Z}_{+} \text {such that } b(n) \neq 0 \text { and } \\
n \equiv-a_{p}\left(M_{1} / p\right) \quad(\bmod p) \text { for all } p \mid M_{1} . "
\end{gathered}
$$

In other words,

$$
\left(a_{p}\right)_{p \mid M_{1}} \notin \prod_{p \mid M_{1}} S_{p} \Longleftrightarrow \begin{gathered}
\text { " } b(n)=0 \text { for all } n \text { 's which satisfy the condi- } \\
M_{1} . "
\end{gathered}
$$

See (1.15) for the definition of $S_{p}$.

\section{$\S 2$. A complete theory of newforms for Kohnen spaces}

We will generalize the results of $\S 4$ of the previous paper [U1] and will get a complete theory of newforms for Kohnen spaces. So we will consider only Kohnen spaces in this and next sections.

We keep to the notations in $\S 0, \S 1$ and in [U1]. See those for the detail of definitions and notations.

We recall some notations.

Let $k$ be a positive integer and $N=4 \times M, M$ is a positive odd integer. Put $\nu_{p}:=\operatorname{ord}_{p}(N)=\operatorname{ord}_{p}(M)$ for any odd prime $p$. We decompose $M=M_{1} M_{2+}, M_{1}:=\prod_{p \mid M, \nu_{p}=1} p$ and $M_{2+}:=\prod_{p \mid M, \nu_{p} \geq 2} p^{\nu_{p}}$.

Let $\chi$ be an even Dirichlet character with $\chi^{2}=1$ and for any prime $p, \chi_{p}$ the $p$-primary component of $\chi$. Moreover we denote the $M_{1}$ (resp. $\left.2 M_{2+}\right)$ component of $\chi$ by $\chi_{1}:=\prod_{p \mid M_{1}} \chi_{p}$ (resp. $\tilde{\chi}:=\prod_{p \mid 2 M_{2+}} \chi_{p}$ ).

We also denote the set of all prime divisors of $M_{2+}$ by $\Pi$. For any $l \in \Pi$, we denote the twisting operator of $\left({ }_{\bar{l}}\right)$ by $R_{l}$ (cf. $\S 0$ (c)). Furthermore for any subset $I \subseteq \Pi$, we put $R_{I}:=\prod_{l \in I} R_{l}$.

For any $\kappa \in\{ \pm 1\}^{\Pi}$ and any $l \in \Pi$, we define operators by $e_{l}^{\kappa}:=$ $\frac{1}{2}\left(R_{l}^{2}+\kappa(l) R_{l}\right)$ and $e_{\Pi}^{\kappa}:=\prod_{l \in \Pi} e_{l}^{\kappa}$. For any subset $I$ of $\Pi$, we set the following notations: $l_{I}:=\prod_{l \in I} l$.

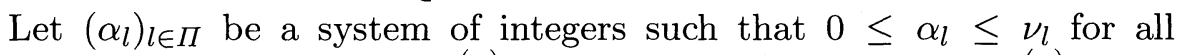
$l \in \Pi$. For simplicity, put $M_{2+}^{(\alpha)}:=\prod_{l \in \Pi} l^{\alpha_{l}}, \tilde{N}(\alpha):=4 M_{1} M_{2+}^{(\alpha)}, D=$ $\Pi(\alpha)_{0}:=\left\{l \in \Pi \mid \alpha_{l}=0\right\}, E=\Pi(\alpha)_{1}:=\left\{l \in \Pi \mid \alpha_{l}=1\right\}$, and 
$F=\Pi(\alpha)_{2+}:=\left\{l \in \Pi \mid \alpha_{l} \geq 2\right\}$. We note that every positive divisor of $M_{2+}$ is of the form $M_{2+}^{(\alpha)}$ for some system $\left(\alpha_{l}\right)_{l \in \Pi \text {. }}$.

We choose and fix $\kappa \in\{ \pm 1\}^{\Pi}$ until the end of this paper. Let $\left.\kappa\right|_{F} \in$ $\{ \pm 1\}^{F}$ be the restriction of $\kappa$ to $F$.

We have to study the subspace $\mathfrak{S}^{\emptyset,\left.\kappa\right|_{F}}(k+1 / 2, \tilde{N}(\alpha), \chi)_{K}$ of $S(k+$ $1 / 2, N, \chi)_{K}$. We recall the definition of this subspace (cf. [U1, (3.3) and $(3.5)])$.

We decompose the character $\chi$ into two parts as follows:

$$
\chi=\eta \eta^{\prime}, \quad \eta=\left(\frac{u}{-}\right), \quad \eta^{\prime}=\left(\frac{u^{\prime}}{-}\right), \quad 0<u\left|l_{D}, \quad 0<u^{\prime}\right| M_{1} l_{E} l_{F} .
$$

Since the character $\eta^{\prime}$ can be defined with modulo $\tilde{N}(\alpha)$, the subspace $\left.\mathfrak{S}^{\emptyset, \kappa^{\prime}}\right|_{F}\left(k+1 / 2, \tilde{N}(\alpha), \eta^{\prime}\right)_{K}$ is naturally defined, where $\left.\kappa^{\prime}\right|_{F}:=\left.\kappa\right|_{F} \cdot(\underline{u}) \in$ $\{ \pm 1\}^{F}$ (cf. [U1, p. 177]). And then, using this subspace, we define as follows

$$
\mathfrak{S}^{\emptyset,\left.\kappa\right|_{F}}(k+1 / 2, \tilde{N}(\alpha), \chi)_{K}:=\mathfrak{S}^{\emptyset,\left.\kappa^{\prime}\right|_{F}}\left(k+1 / 2, \tilde{N}(\alpha), \eta^{\prime}\right)_{K} \mid U(u) .
$$

$\left.\mathfrak{S}^{\emptyset, \kappa^{\prime}}\right|_{F}\left(k+1 / 2, \tilde{N}(\alpha), \eta^{\prime}\right)_{K}$ has a $\mathbf{C}$-basis $\mathcal{B}_{\eta^{\prime}}$ consisting of common eigenforms for all Hecke operators $\tilde{T}\left(n^{2}\right)=\tilde{T}_{k+1 / 2, \tilde{N}(\alpha), \eta^{\prime}}\left(n^{2}\right)\left(n \in \mathbf{Z}_{+},(n, \tilde{N}(\alpha))\right.$ $=1)($ cf. $[\mathrm{U} 1,(3.11)])$. Moreover $U(u)$ gives an injection on $S(k+1 / 2, \tilde{N}(\alpha)$. $\left.l_{D}, \eta^{\prime}\right)_{K}([\mathrm{U} 1,(1.28)])$ and is commutative with all Hecke operators $\tilde{T}\left(n^{2}\right)$ 's (cf. [U1, (1.8) and (1.20)]). Therefore the set $\mathcal{B}_{\chi}:=\left\{g \mid U(u) ; g \in \mathcal{B}_{\eta^{\prime}}\right\}$ gives a C-basis of $\mathfrak{S}^{\emptyset,\left.\kappa\right|_{F}}(k+1 / 2, \tilde{N}(\alpha), \chi)_{K}$ consisting of common eigenforms for all Hecke operators $\tilde{T}\left(n^{2}\right)=\tilde{T}_{k+1 / 2, N, \chi}\left(n^{2}\right)\left(n \in \mathbf{Z}_{+},(n, N)=1\right)$. Put

$$
\begin{aligned}
B^{(\alpha)} & :=\sum_{0<a \mid l_{D}} \mathfrak{S}^{\emptyset, \kappa \mid F}(k+1 / 2, \tilde{N}(\alpha), \chi)_{K} \mid U\left(a^{2}\right) \\
& \subseteq \begin{cases}S(k+1 / 2, N, \chi)_{K} & \text { if } k \geq 2, \\
V(N ; \chi)_{K} & \text { if } k=1 .\end{cases}
\end{aligned}
$$

Then from $[\mathrm{U} 1,(3.10)]$,

$$
\begin{aligned}
B^{(\alpha)} & =\bigoplus_{0<a \mid l_{D}} \mathfrak{S}^{\emptyset,\left.\kappa\right|_{F}}(k+1 / 2, \tilde{N}(\alpha), \chi)_{K} \mid U\left(a^{2}\right) \\
& \subseteq \begin{cases}S^{\emptyset,\left.\kappa\right|_{F}}\left(k+1 / 2, \tilde{N}(\alpha) \cdot l_{D}, \chi\right)_{K} & \text { if } k \geq 2, \\
V^{\emptyset,\left.\kappa\right|_{F}}\left(\tilde{N}(\alpha) \cdot l_{D}, \chi\right)_{K} & \text { if } k=1 .\end{cases}
\end{aligned}
$$


Since every $U\left(a^{2}\right)\left(0<a \mid l_{D}\right)$ gives an injection on $S\left(k+1 / 2, \tilde{N}(\alpha) \cdot l_{D}, \chi\right)$ (cf. [U1, (1.28)]), we get the following:

$$
B^{(\alpha)}=\bigoplus_{f \in \mathcal{B}_{\chi}}\left(\bigoplus_{0<a \mid l_{D}} \mathbf{C} f \mid U\left(a^{2}\right)\right)
$$

where $f$ takes over all elements in $\mathcal{B}_{\chi}$.

Moreover we can see that

$$
B^{(\alpha)} \mid e_{\Pi}^{\kappa} \subseteq \mathfrak{S}^{\emptyset, \kappa}(k+1 / 2, N, \chi)_{K}
$$

In fact, applying the operator $e_{\Pi}^{\kappa}$ to the both sides of the definition, we see that

$$
B^{(\alpha)} \mid e_{\Pi}^{\kappa} \subseteq \begin{cases}S(k+1 / 2, N, \chi)_{K} \mid e_{\Pi}^{\kappa}=S^{\emptyset, \kappa}(k+1 / 2, N, \chi)_{K}, & \text { if } k \geq 2 \\ V(N ; \chi)_{K} \mid e_{\Pi}^{\kappa}=V^{\emptyset, \kappa}(N ; \chi)_{K}, & \text { if } k=1\end{cases}
$$

Every generator of $B^{(\alpha)}\left|e_{\Pi}^{\kappa}, f\right| U\left(a^{2}\right) e_{\Pi}^{\kappa}\left(0<a \mid l_{D}\right)$, is a common eigenform on $\tilde{T}\left(n^{2}\right)\left(n \in \mathbf{Z}_{+},(n, N)=1\right)$ and has the same system of eigen values as $f$ (and so as $g \in \mathcal{B}_{\eta^{\prime}}$ ). This system corresponds to a primitive form in $S^{0}\left(2 k, M_{1} M^{\prime}\right), 0<M^{\prime} \mid M_{2+}^{(\alpha)}$ ([U1, (3.11)]).

From $[\mathrm{U} 1,(3.10)]$ and the Strong Multiplicity One theorem of weight $2 k$ ([M, Theorem 4.6.19]), $\mathfrak{S}^{\emptyset, \kappa}(k+1 / 2, N, \chi)_{K}$ contains all of generators, $f \mid U\left(a^{2}\right) e_{\Pi}^{\kappa}$, and therefore contains all elements of $B^{(\alpha)} \mid e_{\Pi}^{\kappa}$.

We denote for any $f \in \mathcal{B}_{\chi}$

$$
B_{f}^{(\alpha)}:=\bigoplus_{0<a \mid l_{D}} \mathbf{C f} \mid U\left(a^{2}\right)
$$

These spaces $B_{f}^{(\alpha)}$ are contained in $S\left(k+1 / 2, \tilde{N}(\alpha) \cdot l_{D}, \chi\right)$. We will decompose $B_{f}^{(\alpha)}$ by the semi-simple operators $Y_{p}, p \mid M_{1} l_{D} l_{E}$, on $S(k+$ $\left.1 / 2, \tilde{N}(\alpha) \cdot l_{D}, \chi\right)($ cf. $[\mathrm{U} 1,(1.27)])$.

First we consider the case of $p \mid M_{1} l_{E}$.

Take $g \in \mathcal{B}_{\eta^{\prime}}$ such that $f=g \mid U(u)$. Then

$$
g \mid Y_{p} \in \begin{cases}S\left(k+1 / 2, \tilde{N}(\alpha), \eta^{\prime}\right)_{K}, & \text { if } k \geq 2, \\ V\left(\tilde{N}(\alpha) ; \eta^{\prime}\right)_{K}, & \text { if } k=1\end{cases}
$$


and since $R_{l}(l \in F)$ commutes with $Y_{p}([\mathrm{U} 1,(1.24)(2)])$, we see

$$
g \mid Y_{p} \in \begin{cases}S^{\emptyset,\left.\kappa^{\prime}\right|_{F}}\left(k+1 / 2, \tilde{N}(\alpha), \eta^{\prime}\right)_{K}, & \text { if } k \geq 2, \\ V^{\emptyset,\left.\kappa^{\prime}\right|_{F}}\left(\tilde{N}(\alpha) ; \eta^{\prime}\right)_{K}, & \text { if } k=1\end{cases}
$$

Since $Y_{p}$ commutes with Hecke operators $\tilde{T}\left(n^{2}\right),(n, N)=1$, both $g$ and $g \mid Y_{p}$ belong to the same system of eigen values on $\tilde{T}\left(n^{2}\right),(n, N)=$ 1. And since $\left.g \in \mathfrak{S}^{\emptyset, \kappa^{\prime}}\right|_{F}\left(k+1 / 2, \tilde{N}(\alpha), \eta^{\prime}\right)_{K}$, its system of eigenvalues corresponds to a primitive form of weight $2 k$ and of conductor $M_{1} l_{E} \times$ (a divisor of $\left.\prod_{l \in F} l^{\alpha_{l}}\right)([\mathrm{U} 1,(3.10)(2)])$.

From these, first we see that $g\left|Y_{p} \in \mathfrak{S}^{\emptyset, \kappa^{\prime}}\right|_{F}\left(k+1 / 2, \tilde{N}(\alpha) ; \eta^{\prime}\right)_{K}([\mathrm{U} 1$, (3.10) (1)]) and next, by using the Strong Multiplicity One theorem ([U1, (3.11) (2)]), $g \mid Y_{p}=\tau_{p} g$ for some $\tau_{p} \in \mathbf{C}$.

Combining these with Proposition A.1 in Appendix, we obtain that

$$
f\left|Y_{p}=g\right| U(u) Y_{p}=\eta_{p}^{\prime}(u)\left(\frac{u}{p}\right) g \mid Y_{p} U(u)=\eta_{p}^{\prime}(u)\left(\frac{u}{p}\right) \tau_{p} f
$$

i.e., $f$ is an eigen form for $Y_{p}$.

Moreover in this case, $Y_{p}$ commute with $U\left(a^{2}\right)\left(0<a \mid l_{D}\right)$ because of Proposition A.1. Hence we see that every element of $B_{f}^{(\alpha)}$ is a common eigenform on $Y_{p}$ 's $\left(p \mid M_{1} l_{E}\right)$ belonging to the same system of eigen values as $f$.

As to the case of $p \mid l_{D}$, we get the following proposition.

Proposition 6. Let $I$ be any subset of $D$ and $f$ be any element of $\mathcal{B}_{\chi}$. And let $A(I)$ be the subset of $\operatorname{Map}(I, \mathbf{C})$ consisting of all functions $\rho$ such that $\rho(p)= \pm \sqrt{\left(\frac{-1}{p}\right) p}$ resp. $\rho(p) \in\{p,-1\}$ according to $\chi_{p}=\mathbf{1}$ resp. $\left(\frac{}{p}\right)$. Hence $|A(I)|=2^{|I|}$. Then we have the following decomposition:

$$
\bigoplus_{0<a \mid l_{I}} \mathbf{C} f \mid U\left(a^{2}\right)=\bigoplus_{\rho \in A(I)} \mathbf{C} f_{\rho}
$$

where each $f_{\rho}(\rho \in A(I))$ is a non-zero cusp form satisfying the condition:

$$
f_{\rho} \mid Y_{p}=\rho(p) f_{\rho} \quad(\text { for all } p \in I) .
$$

Proof. We will prove by induction on $|I|$.

If $|I|=0(\Leftrightarrow I=\emptyset)$, then $l_{\emptyset}=1$ and the assertion is trivial. 
Next suppose that $I \neq \emptyset$ and the assertion holds good for any proper subset $I^{\prime}$ of $I$.

Take and fix a prime $p \in I$ and decompose $I=\{p\}+I^{\prime}$, i.e. $I^{\prime}=I-\{p\}$. By the assumption of the induction, we have

$$
\bigoplus_{0<a \mid l_{I^{\prime}}} \mathbf{C} f \mid U\left(a^{2}\right)=\bigoplus_{\rho^{\prime} \in A\left(I^{\prime}\right)} \mathbf{C} f_{\rho^{\prime}}
$$

where $A\left(I^{\prime}\right)$ is the subset of $\operatorname{Map}\left(I^{\prime}, \mathbf{C}\right)$ which is described in the assertion. Hence $\left|A\left(I^{\prime}\right)\right|=2^{\left|I^{\prime}\right|}$ and $f_{\rho^{\prime}}\left(\rho^{\prime} \in A\left(I^{\prime}\right)\right)$ is a non-zero cusp form satisfying the condition:

$$
f_{\rho^{\prime}} \mid Y_{p}=\rho^{\prime}(p) f_{\rho^{\prime}} \quad\left(\text { for all } p \in I^{\prime}\right) .
$$

Since $U\left(p^{2}\right)$ gives an automorphism of $S\left(k+1 / 2, \tilde{N}(\alpha) \cdot l_{D}, \chi\right)_{K}$, we can calculate as follows:

$$
\begin{aligned}
\bigoplus_{0<a \mid l_{I}} \mathbf{C} f \mid U\left(a^{2}\right) & =\left(\bigoplus_{0<a \mid l_{I^{\prime}}} \mathbf{C} f \mid U\left(a^{2}\right)\right) \oplus\left(\bigoplus_{0<a \mid l_{I^{\prime}}} \mathbf{C} f \mid U\left(a^{2}\right)\right) \mid U\left(p^{2}\right) \\
& =\left(\bigoplus_{\rho^{\prime} \in A\left(I^{\prime}\right)} \mathbf{C} f_{\rho^{\prime}}\right) \oplus\left(\bigoplus_{\rho^{\prime} \in A\left(I^{\prime}\right)} \mathbf{C} f_{\rho^{\prime}} \mid U\left(p^{2}\right)\right) \\
& =\bigoplus_{\rho^{\prime} \in A\left(I^{\prime}\right)}\left(\mathbf{C} f_{\rho^{\prime}} \oplus \mathbf{C}\left(f_{\rho^{\prime}} \mid U\left(p^{2}\right)\right)\right)
\end{aligned}
$$

From $[\mathrm{U} 1,(1.24)] f_{\rho^{\prime}}\left|U\left(p^{2}\right) Y_{q}=f_{\rho^{\prime}}\right| Y_{q} U\left(p^{2}\right)$ for any $q \in I^{\prime}$. Hence, every element of $\mathbf{C} f_{\rho^{\prime}} \oplus \mathbf{C}\left(f_{\rho^{\prime}} \mid U\left(p^{2}\right)\right)$ is a common eigenform on all $Y_{q}$ ( $\left.q \in I^{\prime}\right)$ which belongs to $\rho^{\prime}$.

Now, we will decompose the space $\mathbf{C} f_{\rho^{\prime}} \oplus \mathbf{C}\left(f_{\rho^{\prime}} \mid U\left(p^{2}\right)\right)$ into two eigen subspaces on $Y_{p}$.

From the definition of $\mathcal{B}_{\chi}$, there exists $g \in \mathcal{B}_{\eta^{\prime}}$ such that $f=g \mid U(u)$. Then

$$
\bigoplus_{0<a \mid l_{I^{\prime}}} \mathbf{C} f\left|U\left(a^{2}\right)=\left(\bigoplus_{0<a \mid l_{I^{\prime}}} \mathbf{C} g \mid U\left(a^{2}\right)\right)\right| U(u)
$$

and so for any $f_{\rho^{\prime}}\left(\rho^{\prime} \in A\left(I^{\prime}\right)\right)$, there exists $g_{\rho^{\prime}} \in \bigoplus_{0<a \mid l_{I^{\prime}}} \mathbf{C} g \mid U\left(a^{2}\right)$ such that $f_{\rho^{\prime}}=g_{\rho^{\prime}} \mid U(u)$.

These forms $g_{\rho^{\prime}}$ satisfy the conditions: $g_{\rho^{\prime}} \in S\left(k+1 / 2, \tilde{N}(\alpha) \cdot l_{I^{\prime}}, \eta^{\prime}\right)$. And also we have $\left(p, \tilde{N}(\alpha) \cdot l_{I^{\prime}}\right)=1,\left(u, 4 l_{F}\right)=1$, and $\eta^{\prime}{ }_{u}=\mathbf{1}$. 
$g$ is an eigen form on $\tilde{T}\left(p^{2}\right)$. From this and [U1, (1.20)], $g_{\rho^{\prime}}$ is also an eigen form on $\tilde{T}\left(p^{2}\right)$ belonging to the same eigen value as $g$.

From the above facts, we can apply the formulae (A.7) and (A.11) in Appendix to the forms $f_{\rho^{\prime}}=g_{\rho^{\prime}} \mid U(u)$ and $f_{\rho^{\prime}}\left|U\left(p^{2}\right)=g_{\rho^{\prime}}\right| U\left(u p^{2}\right)$.

It follows that the space $\mathbf{C} f_{\rho^{\prime}} \oplus \mathbf{C} f_{\rho^{\prime}} \mid U\left(p^{2}\right)$ can be decomposed into two distinct one-dimensional eigen subspaces on $Y_{p}$ :

$$
\mathbf{C} f_{\rho^{\prime}} \oplus \mathbf{C} f_{\rho^{\prime}} \mid U\left(p^{2}\right)=\mathbf{C} h_{1} \oplus \mathbf{C} h_{2} .
$$

And also we see that $h_{1}, h_{2}$ are two common eigenforms on $Y_{q}(q \in I)$ whose eigen values are coincide $\left(=\rho^{\prime}(q)\right)$ on the operators $Y_{q}\left(q \in I^{\prime}\right)$ and are different only on $Y_{p}$. We can extend $\rho^{\prime}$ to two elements in $\operatorname{Map}(I, \mathbf{C})$ by associating $\{p\}$ with each of such two eigen values.

Combining this and the above formula, we obtain the assertion.

From these arguments and Proposition 6, we have the following decomposition:

$$
B_{f}^{(\alpha)}=\bigoplus_{0<a \mid l_{D}} \mathbf{C f} \mid U\left(a^{2}\right)=\bigoplus_{\rho \in A(D)} \mathbf{C} f_{\rho} .
$$

Here $A(D)$ is a subset of $\operatorname{Map}(D, \mathbf{C})$ such that $|A(D)|=2^{|D|}$ and each $f_{\rho}$ is a non-zero common eigen form on $Y_{p}\left(p \mid M_{1} l_{D} l_{E}\right)$ satisfying the condition:

$$
f_{\rho} \mid Y_{p}=c_{p} f_{\rho}, \quad c_{p}:= \begin{cases}\lambda_{p}, & \text { for all } p \mid M_{1} l_{E} \\ \rho(p), & \text { for all } p \in D\end{cases}
$$

where $\lambda_{p}$ is the eigen value of $f \in \mathcal{B}_{\chi}$ on $Y_{p}$.

We take such a $f_{\rho}$ and fix it. Since $f_{\rho} \in S\left(k+1 / 2, \tilde{N}(\alpha) \cdot l_{D}, \chi\right)_{K}$, $h_{\rho}:=f_{\rho} \mid \widetilde{W}\left(M_{1} l_{D} l_{E}\right)^{-1} \in S\left(k+1 / 2, \tilde{N}(\alpha) \cdot l_{D}, \chi\left(\frac{M_{1} l_{D} l_{E}}{}\right)\right)_{K}$ (cf. [U1, $(1.22)])$.

Proposition 7. Let $X_{p}$ be an operator defined in (1.8) of $\S 1$. The above form $h_{\rho}$ is a common eigen form of $X_{p}$ for any $p \mid M_{1} l_{D} l_{E}$.

Proof. From $[\mathrm{U} 1,(1.18)]$ and Proposition A.1, it follows that

$$
\begin{aligned}
h_{\rho} \mid \widetilde{W}\left(M_{1} l_{D} l_{E}\right) U(p) \widetilde{W}(p) & =c_{0} h_{\rho} \mid \widetilde{W}(p) \widetilde{W}\left(M_{1} l_{D} l_{E} / p\right) U(p) \widetilde{W}(p) \\
& =h_{\rho} \mid \widetilde{W}(p) U(p) \widetilde{W}\left(M_{1} l_{D} l_{E} / p\right) \widetilde{W}(p) \\
& =c_{1} h_{\rho} \mid \widetilde{W}(p) U(p) \widetilde{W}\left(M_{1} l_{D} l_{E}\right),
\end{aligned}
$$


where $c_{0}=\left(\chi\left(\frac{M_{1} l_{D} l_{E}}{}\right)\right)_{M_{1} l_{D} l_{E} / p}(p)$ and $c_{1}=\left(\chi\left(\frac{M_{1} l_{D} l_{E}}{}\right)\right)_{p}\left(M_{1} l_{D} l_{E} / p\right)$.

Hence, for any prime divisor $p$ of $M_{1} l_{D} l_{E}$,

$$
\begin{aligned}
c_{p} h_{\rho} & =f_{\rho} \mid Y_{p} \widetilde{W}\left(M_{1} l_{D} l_{E}\right)^{-1} \\
& =p^{-k / 2+3 / 4} h_{\rho} \mid \widetilde{W}\left(M_{1} l_{D} l_{E}\right) U(p) \widetilde{W}(p) \widetilde{W}\left(M_{1} l_{D} l_{E}\right)^{-1} \\
& =c_{1} h_{\rho} \mid X_{p}
\end{aligned}
$$

Thus we proved the above claim.

From this claim and Proposition 5, $\Delta_{0}\left(4 \prod_{l \in F} l^{\alpha_{l}}\right) / \Delta\left(4 M_{1} l_{D} M_{2+}^{(\alpha)}\right)$ module $\boldsymbol{\pi}_{h_{\rho}}$ is irreducible. We decompose it into primary components:

$$
\pi_{h_{\rho}} \cong \underline{\underline{\psi}} \otimes\left(\bigotimes_{p \mid M_{1}} \theta_{p}\right) \otimes\left(\bigotimes_{p \mid l_{D} l_{E}} \theta_{p}\right)
$$

where $\psi=\prod_{p \nmid M_{1} l_{D} l_{E}}\left(\chi\left(\frac{M_{1} l_{D} l_{E}}{}\right)\right)_{p}$ and $\underline{\underline{\psi}}$ is defined by $\psi$ in the same way as (1.5).

Proposition 8. We can determine each primary component $\theta_{p}(p \in$ $D+E)$ by using (1.12) and (2.9) as follows:

[Case 1] $\left(p \mid l_{D} l_{E}\right.$ and $\left.\chi_{p}=\mathbf{1}\right)$

$$
\theta_{p} \cong \begin{cases}\mathfrak{C}_{(p+1) / 2}, & \text { if } \lambda_{p}=\left(\frac{M_{1} l_{D} l_{E} / p}{p}\right) \mathfrak{g}_{p} \text { and } p \in E \\ \mathfrak{C}_{(p+1) / 2}^{\prime}, & \text { if } \lambda_{p}=-\left(\frac{M_{1} l_{D} l_{E} / p}{p}\right) \mathfrak{g}_{p} \text { and } p \in E \\ \mathfrak{C}_{(p+1) / 2}, & \text { if } \rho(p)=\left(\frac{M_{1} l_{D} l_{E} / p}{p}\right) \mathfrak{g}_{p} \text { and } p \in D \\ \mathfrak{C}_{(p+1) / 2}^{\prime}, & \text { if } \rho(p)=-\left(\frac{M_{1} l_{D} l_{E} / p}{p}\right) \mathfrak{g}_{p} \text { and } p \in D\end{cases}
$$

[Case 2] $\left(p \mid l_{D}\right.$ and $\left.\chi_{p}:(\bar{p})\right)$

$$
\theta_{p} \cong \begin{cases}\mathbf{1}_{\mathbf{G}(p)}, & \text { if } \rho(p)=p \\ \mathfrak{C}_{p}, & \text { if } \rho(p)=-1\end{cases}
$$

$\left[\right.$ Case 3] $\left(p \mid l_{E}\right.$ and $\left.\chi_{p}=(\bar{p})\right) \quad \theta_{p} \cong \mathfrak{C}_{p}$. 
Proof. The assertions in Cases 1 and 2 are easily verified.

We consider Case 3. Suppose that $\theta_{p} \cong \mathbf{1}_{\mathbf{G}(p)}$. We have $h_{\rho} \mid X_{p}=p h_{\rho}$ from Proposition $5(2)$. Hence $\lambda_{p}=p$. This follows from (2.8) and (2.9). Moreover, using (2.6) and the fact: $\eta_{p}^{\prime}=\chi_{p}=\left(\frac{-}{p}\right)$, we get that $g \mid Y_{p}=p g$.

Applying $[\mathrm{U} 1,(1.26)]$ to $g\left(=\sum_{n \geq 1} a(n) \mathbf{e}(n z) \in S\left(k+1 / 2, \tilde{N}(\alpha), \eta^{\prime}\right)_{K}\right)$ and $p$, we have $g\left|Y_{p}=g\right| \widetilde{W}(p)^{-1} \tilde{\delta}_{p}-g+p^{-k / 2+3 / 4} g \mid U(p) \tilde{\delta}_{p}$.

Since $p^{-k / 2+3 / 4} g\left|U(p)=\left(g \mid Y_{p}\right)\right| \widetilde{W}(p)^{-1}=(p g) \mid \widetilde{W}(p)^{-1}$, we therefore have $g=p^{-k / 2-1 / 4} g \mid U(p) \tilde{\delta}_{p}=\sum_{n \geq 1} a(p n) \mathbf{e}(p n z)$.

From this formula we have that $a(n)=0$ if $(p, n)=1$. Hence there is a form $g^{\prime} \in S\left(k+1 / 2, \tilde{N}(\alpha) / p, \eta^{\prime}(\underline{p})\right)_{K}$ if $k \geq 2$, resp. $V\left(\tilde{N}(\alpha) / p ; \eta^{\prime}(\underline{p})\right)_{K}$ if $k=1$ such that $g=g^{\prime}(p z)([\mathrm{U} 1,(1.11)])$.

Both $g$ and $g^{\prime}$ have the same system of eigen values on the Hecke operators $\tilde{T}\left(n^{2}\right),(n, N)=1$ (cf. [U1, (1.8)]). Since $g$ is in $\mathfrak{S}^{\emptyset, \kappa^{\prime} \mid F}(k+$ $\left.1 / 2, \tilde{N}(\alpha), \eta^{\prime}\right)_{K}$, the system of eigen values corresponds to a primitive forms of weight $2 k$ and of conductor $M_{1} l_{E} \times\left(\right.$ a divisor of $\left.\prod_{l \in F} l^{\alpha_{l}}\right)$.

On the other hand, from [U1, (3.10)] for the spaces $S(k+1 / 2, \tilde{N}(\alpha) / p$, $\left.\eta^{\prime}(\underline{p})\right)_{K}$ and $V\left(\tilde{N}(\alpha) / p ; \eta^{\prime}(\underline{p})\right)_{K}$, the system of eigen values of $g^{\prime}$ corresponds to a primitive form of weight $2 k$ and of a conductor prime to $p$.

This is a contradiction. Hence we have $\theta_{p} \cong \mathfrak{C}_{p}$.

Now we will find the condition for $f_{\rho} \mid e_{I I}^{\kappa} \neq 0$ by using the results in $\S 1$.

Proposition 9. (1) If $\chi_{p}=\mathbf{1}$, we have $\lambda_{p}= \pm \mathfrak{g}_{p}(p \in E)$ and $\rho(p)= \pm \mathfrak{g}_{p}(p \in D)$. If $\chi_{p}=\left(\frac{-}{p}\right), \rho(p)=p$ or $-1(p \in D)$.

(2) We have

$$
f_{\rho} \mid e_{\Pi}^{\kappa} \neq 0 \Longleftrightarrow \begin{cases}\lambda_{p}=\left(\frac{-1}{p}\right) \kappa(p) \mathfrak{g}_{p}, & \text { for all } p \in E \text { with } \chi_{p}=\mathbf{1} \\ \rho(p)=-1, & \text { for all } p \in D \text { with } \chi_{p}=\left(\frac{-}{p}\right), \\ \rho(p)=\left(\frac{-1}{p}\right) \kappa(p) \mathfrak{g}_{p}, & \text { for all } p \in D \text { with } \chi_{p}=\mathbf{1}\end{cases}
$$
on $f$.

Remark. In the above, the condition: $\lambda_{p}=\left(\frac{-1}{p}\right) \kappa(p) \mathfrak{g}_{p}$ depends only

Proof. The first assertion follows from [U1, (1.27)] and (2.8). We will prove the second assertion. 
$[\Rightarrow]$ We apply Theorem 1 to the forms $h_{\rho}$ and $f_{\rho}(\in S(k+1 / 2, \tilde{N}(\alpha) \cdot$ $\left.\left.l_{D}, \chi\right)_{K}\right)$. Set $f_{\rho}(z):=\sum_{n \geq 1} c(n) \mathbf{e}(n z)$ and use the notation $S_{p}$ on $\boldsymbol{\pi}_{h_{\rho}}$ in (1.15).

From Theorem 1, we know the following:

$$
c(n)=0 \quad \text { if } n \equiv-x\left(M_{1} l_{D} l_{E} / p\right) \bmod p \text { for some } x \bmod p \notin S_{p} .
$$

We modify the condition. Let first $p \in E$ with $\chi_{p}=\mathbf{1}$. From the definition of $S_{p}$ and Proposition 8,

the condition in $(2.11) \Longleftrightarrow-n\left(M_{1} l_{D} l_{E} / p\right)^{-1} \quad(\bmod p) \notin S_{p}$

$$
\Longleftrightarrow\left(\frac{-n M_{1} l_{D} l_{E} / p}{p}\right)= \begin{cases}-1, & \text { if } \theta_{p} \cong \mathfrak{C}_{(p+1) / 2}, \\ 1, & \text { if } \theta_{p} \cong \mathfrak{C}_{(p+1) / 2}^{\prime} .\end{cases}
$$

Suppose that $\theta_{p} \cong \mathfrak{C}_{(p+1) / 2}$. From the first assertion, $\lambda_{p}= \pm \mathfrak{g}_{p}$. If $\lambda_{p}=-\left(\frac{M_{1} l_{D} l_{E} / p}{p}\right) \mathfrak{g}_{p}$, then $\theta_{p} \cong \mathfrak{C}_{(p+1) / 2}^{\prime}$ (cf. Proposition 8). This is a contradiction. Hence $\lambda_{p}=\left(\frac{M_{1} l_{D} l_{E} / p}{p}\right) \mathfrak{g}_{p}$ and so $\left(\frac{-n}{p}\right) \lambda_{p} \mathfrak{g}_{p}^{-1}=-1$. Thus we can easily deduce the following from similar argument.

$$
\text { the condition in } \begin{aligned}
(2.11) & \Longleftrightarrow\left(\frac{-n}{p}\right) \lambda_{p} \mathfrak{g}_{p}^{-1}=-1 \\
& \Longleftrightarrow\left(\frac{n}{p}\right)=-\left(\frac{-1}{p}\right) \mathfrak{g}_{p} \lambda_{p}^{-1} .
\end{aligned}
$$

We suppose that $\lambda_{p}=-\left(\frac{-1}{p}\right) \kappa(p) \mathfrak{g}_{p}\left(\Leftrightarrow \kappa(p)=-\left(\frac{-1}{p}\right) \mathfrak{g}_{p} \lambda_{p}{ }^{-1}\right)$ for some $p \in E$ with $\chi_{p}=\mathbf{1}$. If $\left(\frac{n}{p}\right)=\kappa(p)$, we have $c(n)=0$ from (2.12). On the other hand, if $\left(\frac{n}{p}\right)=-\kappa(p)$ or 0 , we have $\left(\frac{n}{p}\right)^{2}+\kappa(p)\left(\frac{n}{p}\right)=0$.

Observing that $f_{\rho} \mid e_{p}^{\kappa}=\frac{1}{2} \sum_{n \geq 1} c(n)\left(\left(\frac{n}{p}\right)^{2}+\kappa(p)\left(\frac{n}{p}\right)\right) \mathbf{e}(n z)$, we get $f_{\rho} \mid e_{p}^{\kappa}=0$ and so $f_{\rho} \mid e_{\Pi}^{\kappa}=0$.

In the same way, we get the assertions in the case of $p \in D$ with $\chi_{p}=\mathbf{1}$.

Next we consider the case of $p \in D$ with $\chi_{p}=\left(\frac{\bar{p}}{)}\right.$. Let $\theta_{p} \cong \mathbf{1}_{\mathbf{G}(p)}$. Then $S_{p}=\{0 \bmod p\}$. It follows from $(2.11)$ that $c(n)=0$ for any $n$ prime to $p$. Hence we have $f_{\rho} \mid e_{p}^{\kappa}=0$. From these $f_{\rho} \mid e_{\Pi}^{\kappa} \neq 0 \Rightarrow \theta_{p} \cong \mathfrak{C}_{p} \Rightarrow$ $\rho(p)=-1$. We use the first assertion at the last implication. 
$[\Leftarrow]$ From $(2.2)$ and $f_{\rho} \in B_{f}^{(\alpha)} \subseteq B^{(\alpha)}$, it follows that $f_{\rho} \mid R_{p}=\kappa(p) f_{\rho}$ for all $p \in F$. Hence for all $p \in F, f_{\rho} \mid e_{p}^{\kappa}=f_{\rho}$. Therefore,

$$
\begin{aligned}
f_{\rho} \mid e_{\Pi}^{\kappa} & =f_{\rho} \mid \prod_{p \in D+E} e_{p}^{\kappa} \\
& =2^{-(|D|+|E|)} \sum_{n \geq 1} c(n) \prod_{p \in D+E}\left\{\left(\frac{n}{p}\right)^{2}+\kappa(p)\left(\frac{n}{p}\right)\right\} \mathbf{e}(n z) .
\end{aligned}
$$

For each prime $p \mid M_{1} l_{D} l_{E}$, we choose a residue class $b_{p} \quad(\bmod p) \in \mathbf{F}_{p}^{\times}$ satisfying the following condition:

$$
\begin{cases}\left(\frac{b_{p}}{p}\right)=\left(\frac{M_{1} l_{D} l_{E} / p}{p}\right) \mathfrak{g}_{p} \lambda_{p}{ }^{-1}, & \text { if } p \in E \text { and } \chi_{p}=\mathbf{1}, \\ \left(\frac{b_{p}}{p}\right)=\left(\frac{M_{1} l_{D} l_{E} / p}{p}\right) \mathfrak{g}_{p} \rho(p)^{-1}, & \text { if } p \in D \text { and } \chi_{p}=\mathbf{1}, \\ \left(\frac{b_{p}}{p}\right)=\left(\frac{-1}{p}\right)\left(\frac{M_{1} l_{D} l_{E} / p}{p}\right) \kappa(p), & \text { if } p \in D+E \text { and } \chi_{p}=(\bar{p}), \\ b_{p} \in S_{p}, & \text { if } p \mid M_{1} .\end{cases}
$$

We can see from the definition $S_{p}$ and Proposition 8 that $\left(b_{p}\right)_{p \mid M_{1} l_{D} l_{E}} \in$ $\prod_{p \mid M_{1} l_{D} l_{E}} S_{p}$. Hence by using Theorem 1 , there exists $n_{0} \in \mathbf{Z}_{+}$such that $c\left(n_{0}\right) \neq 0$ and $-n_{0} \equiv b_{p}\left(M_{1} l_{D} l_{E} / p\right) \quad(\bmod p)$ for all prime divisors $p$ of $M_{1} l_{D} l_{E}$.

For such a $n_{0}$, we have by using the assumption

$$
\begin{aligned}
\left(\frac{n_{0}}{p}\right)= & \left(\frac{-1}{p}\right)\left(\frac{b_{p}}{p}\right)\left(\frac{M_{1} l_{D} l_{E} / p}{p}\right) \\
= & \begin{cases}\left(\frac{-1}{p}\right) \mathfrak{g}_{p} \lambda_{p}^{-1}=\kappa(p), & \text { if } p \in E \text { and } \chi_{p}=\mathbf{1}, \\
\left(\frac{-1}{p}\right) \mathfrak{g}_{p} \rho(p)^{-1}=\kappa(p), & \text { if } p \in D \text { and } \chi_{p}=\mathbf{1}, \\
\kappa(p), & \text { if } p \in D+E \text { and } \chi_{p}=(\bar{p}) .\end{cases}
\end{aligned}
$$

Thus we see that $\left(\frac{n_{0}}{p}\right)=\kappa(p)$ and also that the $n_{0}$-th Fourier coefficient of $f_{\rho} \mid e_{\Pi}^{\kappa}$ is equal to $2^{-(|D|+|E|)} c\left(n_{0}\right) \prod_{p \in D+E}\left\{\kappa(p)^{2}+\kappa(p) \kappa(p)\right\}=c\left(n_{0}\right) \neq 0$. This means that $f_{\rho} \mid e_{\Pi}^{\kappa} \neq 0$.

Combining (2.5), (2.7) with Proposition 9, we get the following proposition. 
Proposition 10. Let the notation be the same as above. Then

$$
\begin{aligned}
B_{f}^{(\alpha)} \mid e_{\Pi}^{\kappa} & =\mathbf{C} f_{\rho_{0}} \mid e_{\Pi I}^{\kappa}, \\
\rho_{0}(p) & := \begin{cases}\left(\frac{-1}{p}\right) \kappa(p) \mathfrak{g}_{p}, & \text { for } p \in D \text { and } \chi_{p}=\mathbf{1}, \\
-1, & \text { for } p \in D \text { and } \chi_{p}=(\bar{p}) .\end{cases}
\end{aligned}
$$

Moreover $f_{\rho_{0}} \mid e_{\Pi}^{\kappa} \neq 0$ if and only if $f \in \mathcal{B}_{\chi}$ satisfies the following conditions:

$$
f \mid Y_{p}=\left(\frac{-1}{p}\right) \kappa(p) \mathfrak{g}_{p} f \quad \text { for all } p \in E \text { with } \chi_{p}=\mathbf{1}
$$

We have the following formula:

Proposition 11.

$$
\begin{aligned}
& B^{(\alpha)}\left|e_{\Pi}^{\kappa}=\bigoplus_{f \in \mathcal{B}_{\chi}, f:(*)} \mathbf{C} f_{\rho_{0}}\right| e_{\Pi}^{\kappa}, \\
& \text { and } \rho_{0}(p):= \begin{cases}\left(\frac{-1}{p}\right) \kappa(p) \mathfrak{g}_{p}, & \text { for } p \in D \text { and } \chi_{p}=\mathbf{1}, \\
-1, & \text { for } p \in D \text { and } \chi_{p}=(\bar{p}),\end{cases}
\end{aligned}
$$

where $(*)$ is the same condition as in Proposition 10 and $f_{\rho_{0}}$ is a non-zero common eigenform on $Y_{p}\left(p \mid M_{1} l_{D} l_{E}\right)$ such that $f_{\rho_{0}} \mid Y_{p}=\rho_{0}(p) f_{\rho_{0}}$ if $p \in D$ resp. $\lambda_{p} f_{\rho_{0}}$ if $p \in E$. Here $\lambda_{p}$ is the eigen value of $f$ on $Y_{p}$.

Proof. From $[\mathrm{U} 1,(1.20)]$ and $[\mathrm{U} 3,(1.7)], f_{\rho_{0}} \mid e_{\Pi}^{\kappa} \in B_{f}^{(\alpha)}$ is a common eigenform on all $\tilde{T}\left(n^{2}\right)((n, N)=1)$ belonging to the same system of eigenvalues as that of $f$.

And since there is a $g \in \mathcal{B}_{\eta^{\prime}}$ such that $f=g \mid U(u)$, the system of eigenvalues is also the same as those of $g \in \mathcal{B}_{\eta^{\prime}}$.

By applying the Strong Multiplicity One theorem [U1, (3.11) (2)] to elements in $\mathcal{B}_{\eta^{\prime}}$, each different element in $\mathcal{B}_{\eta^{\prime}}$ belongs to a different system of eigenvalues from each other. Therefore, $\left\{f_{\rho_{0}} \mid e_{\Pi}^{\kappa} ; f \in \mathcal{B}_{\chi}, f\right.$ satisfies the condition $(*)\}$ is a system of linear independent forms.

We will express the above condition $(*)$ in terms of $g \in \mathcal{B}_{\eta^{\prime}}$ such that $f=g \mid U(u)$. Since $\eta_{p}^{\prime}=\chi_{p}$ for all $p \in E$, we have $f\left|Y_{p}=\left(\frac{u}{p}\right) g\right| Y_{p} U(u)$ for all $p \in E$ with $\chi_{p}=\mathbf{1}$ (cf. (2.6)). 
Hence, the condition $(*) \Leftrightarrow\left(\frac{u}{p}\right) g\left|Y_{p} U(u)=\left(\frac{-1}{p}\right) \kappa(p) \mathfrak{g}_{p} g\right| U(u)$.

Moreover since $U(u)$ is an isomorphism on $S\left(k+1 / 2, \tilde{N}(\alpha) \cdot l_{D}, \eta^{\prime}\right)$, we get that the condition $(*) \Leftrightarrow g \mid Y_{p}=\left(\frac{-u}{p}\right) \kappa(p) \mathfrak{g}_{p} g$.

In $[\mathrm{U} 1,(3.6)]$, we defined the hermitian involutions $\mathbf{w}_{p}\left(p \mid M_{1} l_{E}\right)$ on $\mathfrak{S}^{\emptyset,\left.\kappa^{\prime}\right|_{F}}\left(k+1 / 2, \tilde{N}(\alpha), \eta^{\prime}\right)_{K}$. Then $g\left|\mathbf{w}_{p}=p^{-1 / 2}\left(\frac{-1}{p}\right)^{k+1 / 2} \eta^{\prime}(p) g\right| Y_{p}$ for all $p \in E$ with $\chi_{p}=1$. This follows from [U1, (3.7)] and $\eta_{p}^{\prime}=\chi_{p}$ for all $p \in$ $E$. Hence $f:(*) \Leftrightarrow g \mid \mathbf{w}_{p}=\left(\frac{-1}{p}\right)^{k} \eta^{\prime}(p)\left(\frac{u}{p}\right) \kappa(p) g=\left(\frac{-1}{p}\right)^{k} \chi(p) \kappa(p) g$. Here, we note that $\chi(p)$ is meaningful because of $\chi_{p}=\mathbf{1}$.

We denote this condition for $g$ by $(* *)$ :

$$
g \mid \mathbf{w}_{p}=\left(\frac{-1}{p}\right)^{k} \chi(p) \kappa(p) g \text { for all } p \in E \text { with } \chi_{p}=\mathbf{1}
$$

and we denote

$$
\begin{aligned}
\mathfrak{S}_{* *}^{\emptyset,\left.\kappa^{\prime}\right|_{F}} & =\mathfrak{S}_{* *}^{\emptyset,\left.\kappa^{\prime}\right|_{F}}\left(k+1 / 2, \tilde{N}(\alpha), \eta^{\prime}\right)_{K} \\
& :=\left\{\begin{array}{l}
h \in \mathfrak{S}^{\emptyset,\left.\kappa^{\prime}\right|_{F}}\left(k+1 / 2, \tilde{N}(\alpha), \eta^{\prime}\right)_{K} ; \\
h \mid \mathbf{w}_{p}=\left(\frac{-1}{p}\right)^{k} \chi(p) \kappa(p) h \text { for all } p \in E \text { with } \chi_{p}=\mathbf{1}
\end{array}\right\} .
\end{aligned}
$$

From [U1, (3.9) and (3.11)], we have $g \mid \mathbf{w}_{p}= \pm g$ for all $g \in \mathcal{B}_{\eta^{\prime}}$ and so $\left\{g \in \mathcal{B}_{\eta^{\prime}} ; g\right.$ satisfies the condition $\left.(* *)\right\}$ is a basis of the space $\mathfrak{S}_{* *}^{\emptyset,\left.\kappa^{\prime}\right|_{F}}$.

As we show in the proof of Proposition 11, all of three forms $f_{\rho_{0}} \mid e_{\Pi}^{\kappa}$, $f$, and $g$ are common eigenforms on $\tilde{T}\left(n^{2}\right)\left(n \in \mathbf{Z}_{+},(n, N)=1\right)$ and all of them belong to the same system of eigenvalues.

Therefore, for all $n \in \mathbf{Z}_{+}$and $(n, N)=1$,

$$
\begin{aligned}
\operatorname{tr}\left(\tilde{T}\left(n^{2}\right) ; B^{(\alpha)} \mid e_{\Pi}^{\kappa}\right) & =\operatorname{tr}\left(\tilde{T}\left(n^{2}\right) ; \bigoplus_{f \in \mathcal{B}_{\chi}, f:(*)} \mathbf{C} f_{\rho_{0}} \mid e_{\Pi}^{\kappa}\right) \\
& =\operatorname{tr}\left(\tilde{T}\left(n^{2}\right) ; \bigoplus_{g \in \mathcal{B}_{\eta^{\prime}, g:(* *)}} \mathbf{C} g\right) \\
& =\operatorname{tr}\left(\tilde{T}\left(n^{2}\right) ;\left.\mathfrak{S}_{* *}^{\emptyset, \kappa^{\prime}}\right|_{F}\left(k+1 / 2, \tilde{N}(\alpha), \eta^{\prime}\right)_{K}\right)
\end{aligned}
$$

We will describe the right hand side of the above formula in terms of cusp forms of weight $2 k$. 
Take any element $g$ of the basis of $\left.\mathfrak{S}_{* *}^{\emptyset, \kappa^{\prime}}\right|_{F}$, i.e. $g \in \mathcal{B}_{\eta^{\prime}}$ which satisfies the condition $(* *)$. Let $G$ be the primitive form of weight $2 k$ which corresponds to $g$ in the sense of $[\mathrm{U} 1$, Theorem (3.11)]. From [U1, (3.9)], we have $g$ | $U\left(p^{2}\right)=-p^{k-1} g \mid \mathbf{w}_{p}=-p^{k-1}\left(\frac{-1}{p}\right)^{k} \chi(p) \kappa(p) g$ for all $p \in E$ with $\chi_{p}=\mathbf{1}$. So from $[\mathrm{U} 1,(3.11)], G \mid U(p)=-p^{k-1}\left(\frac{-1}{p}\right)^{k} \chi(p) \kappa(p) G$.

Let $W(p)$ be the Atkin-Lehner operator of weight $2 k$ (cf. $\S 0(\mathrm{~b}))$. From [M, Corollary 4.6.18], $G\left|U(p)=-p^{k-1} G\right| W(p)$ and so $G \mid W(p)=$ $\left(\frac{-1}{p}\right)^{k} \chi(p) \kappa(p) G$ for all $p \in E$ with $\chi_{p}=\mathbf{1}$. Hence the space $\mathfrak{S}_{* *}^{\emptyset,\left.\kappa^{\prime}\right|_{F}}$ corresponds to all such $G$ 's.

We can deduce the following expression by the trace relation in the paper [U1, (3.10) (2), (A.2) (3), and (A.5)]:

For any $n \in \mathbf{Z}_{+}$prime to $\tilde{N}(\alpha)$,

$$
\begin{aligned}
& \operatorname{tr}\left(\tilde{T}\left(n^{2}\right) ; \mathfrak{S}_{* *}^{\emptyset,\left.\kappa^{\prime}\right|_{F}}\left(k+1 / 2, \tilde{N}(\alpha), \eta^{\prime}\right)_{K}\right) \\
& =\sum_{\left(\left(\beta_{l}\right), \Psi^{\prime},\left(\tau^{\prime}, \sigma^{\prime}\right)\right) \in P^{\prime}} \Xi^{\prime}\left(\left(\beta_{l}\right), \Psi^{\prime},\left(\tau^{\prime}, \sigma^{\prime}\right)\right) \\
& \quad \times \operatorname{tr}\left(T(n) ; S^{*\left(\hat{\tau}^{\prime}, \sigma^{\prime}\right)}\left(2 k, M_{1} l_{E} \prod_{l \in F} l^{\beta_{l}}\right) \mid R_{\Psi^{\prime}}\right),
\end{aligned}
$$

where the notations are as follows: $\sum_{\left(\left(\beta_{l}\right), \Psi^{\prime},\left(\tau^{\prime}, \sigma^{\prime}\right)\right) \in P^{\prime}}$ is the sum extended over all elements of the following set:

$$
P^{\prime}:=\left\{\begin{array}{l}
\left(\left(\beta_{l}\right)_{l \in F}, \Psi^{\prime},\left(\tau^{\prime}, \sigma^{\prime}\right)\right) ;\left(\beta_{l}\right)=\left(\beta_{l}\right)_{l \in F} \text { is a system of integers } \\
\operatorname{such} \text { that } 0 \leq \beta_{l} \leq \alpha_{l} \text { for any } l \in F, \Psi^{\prime} \subseteq F(\beta)_{0}+F(\beta)_{1}, \\
\tau^{\prime} \in \operatorname{Map}(F,\{ \pm 1\}), \sigma^{\prime} \in \operatorname{Map}\left(F(\beta)_{2+},\{ \pm 1\}\right)
\end{array}\right\} .
$$

$F(\beta)_{i}:=\left\{l \in F \mid \beta_{l}=i\right\}$ for $i=0,1$ and $F(\beta)_{2+}:=\left\{l \in F \mid \beta_{l} \geq 2\right\}$. $\Xi^{\prime}\left(\left(\beta_{l}\right), \Psi^{\prime},\left(\tau^{\prime}, \sigma^{\prime}\right)\right)=\prod_{p \in F} \Xi_{p}^{\prime}\left(\left(\beta_{l}\right), \Psi^{\prime},\left(\tau^{\prime}, \sigma^{\prime}\right)\right)$. Each $\Xi_{p}^{\prime}\left(\left(\beta_{l}\right), \Psi^{\prime},\left(\tau^{\prime}, \sigma^{\prime}\right)\right)$ is the constant determined by the table $[\mathrm{U} 1,(2.22)] . \hat{\tau}^{\prime}$ is the extension of $\tau^{\prime}$ which is defined as follows:

$$
\hat{\tau}^{\prime}(l)= \begin{cases}\tau^{\prime}(l), & \text { if } l \in F \\ \left(\frac{-1}{l}\right)^{k} \chi(l) \kappa(l) \prod_{q \in \Psi^{\prime}}\left(\frac{l}{q}\right), & \text { if } l \in E \text { and } \chi_{l}=\mathbf{1}\end{cases}
$$


Finally,

$$
S^{*\left(\hat{\tau}^{\prime}, \sigma^{\prime}\right)}\left(2 k, M_{1} l_{E} \prod_{l \in F} l^{\beta_{l}}\right):=\left\{\begin{array}{l}
f \in S^{*}\left(2 k, M_{1} l_{E} \prod_{l \in F} l^{\beta_{l}}\right) ; f \mid W_{l}= \\
\hat{\tau}^{\prime}(l) f \text { for all } l \in F+\left\{l \in E \mid \chi_{l}=\right. \\
\mathbf{1}\}, f\left|R_{l} W_{l}=\sigma^{\prime}(l) f\right| R_{l} \text { for all } \\
l \in F(\beta)_{2+}
\end{array}\right\}
$$

See [U1, Appendix 1] for the definition of the space $S^{*}\left(2 k, M_{1} l_{E} \prod_{l \in F} l^{\beta_{l}}\right)$.

In order to compare this formula (2.14) with those in $[U 1,(3.10)(2)]$, we must modify the above formula.

We define a system of integers $\left(\tilde{\beta}_{l}\right)_{l \in \Pi}$ for each system of integers $\left(\beta_{l}\right)_{l \in F}$ as follows: $\tilde{\beta}_{l}=\beta_{l}, 1$, or 0 according to $l \in F, E$, or $D$.

Then we have $\Pi(\tilde{\beta})_{0}=D+F(\beta)_{0}, \Pi(\tilde{\beta})_{1}=E+F(\beta)_{1}, \Pi(\tilde{\beta})_{2+}=$ $F(\beta)_{2+}$, and $l_{E} \times \prod_{l \in F} l^{\beta_{l}}=\prod_{l \in \Pi} l^{\tilde{\beta}_{l}}$.

We want to extend the domain of definition of $\tau^{\prime}(=F)$ to the whole set $\Pi$. Since $\{ \pm 1\}^{\Pi}=\{ \pm 1\}^{F} \times\{ \pm 1\}^{D+E}$, we will append this factor $\{ \pm 1\}^{D+E}$ and denote each element of $\{ \pm 1\}^{\Pi}=\{ \pm 1\}^{F} \times\{ \pm 1\}^{D+E}$ as $\tau=\left(\tau^{\prime}, \tau^{\prime \prime}\right)$.

We note that there are some assumptions on the primes $l \in E$ with $\chi_{l}=\mathbf{1}$ in the definition of the space $S^{*\left(\hat{\tau}^{\prime}, \sigma^{\prime}\right)}\left(2 k, M_{1} l_{E} \prod_{l \in F} l^{\beta_{l}}\right)$. We remove this condition by replacing the coefficients $\Xi^{\prime}\left(\left(\beta_{l}\right), \Psi^{\prime},\left(\tau^{\prime}, \sigma^{\prime}\right)\right)=$ : $\prod_{p \in F} \Xi_{p}^{\prime}\left(\left(\beta_{l}\right), \Psi^{\prime},\left(\tau^{\prime}, \sigma^{\prime}\right)\right)$ with $\prod_{p \in F} \Xi_{p}^{\prime}\left(\left(\beta_{l}\right), \Psi^{\prime},\left(\tau^{\prime}, \sigma^{\prime}\right)\right) \times \prod_{p \in D+E} \Xi_{p}^{\prime \prime}$, where the notation is as follows:

$$
2 \times \Xi_{p}^{\prime \prime}:= \begin{cases}1+\tau^{\prime \prime}(p), & \text { if } p \in D ; \\ 1+1, & \text { if } p \in E \text { and } \chi_{p}=\left(\frac{-}{p}\right) \\ 1+\left(\frac{-1}{p}\right)^{k} \chi(p) \kappa(p) \prod_{q \in \Psi^{\prime}}\left(\frac{p}{q}\right) \tau^{\prime \prime}(p), & \text { if } p \in E \text { and } \chi_{p}=\mathbf{1} .\end{cases}
$$

Finally replacing $\sigma^{\prime}$ with $\sigma$ and combining the formula (2.13) and (2.14), we can obtain the following expression of $\operatorname{tr}\left(\tilde{T}\left(n^{2}\right) ; B^{(\alpha)} \mid e_{\Pi}^{\kappa}\right)$ in terms of primitive forms of weight $2 k$ :

Under the above notation, we get the followings for all $n \in \mathbf{Z}_{+}$prime to $N$,

$$
\begin{aligned}
& \operatorname{tr}\left(\tilde{T}\left(n^{2}\right) ; B^{(\alpha)} \mid e_{I I}^{\kappa}\right) \\
& \quad=\sum_{\left(\left(\beta_{l}\right), \Psi^{\prime},\left(\tau^{\prime}, \sigma\right), \tau^{\prime \prime}\right) \in P^{\prime} \times\{ \pm 1\}^{D+E}} \prod_{p \in F} \Xi_{p}^{\prime}\left(\left(\beta_{l}\right), \Psi^{\prime},\left(\tau^{\prime}, \sigma\right)\right) \times \prod_{p \in D+E} \Xi_{p}^{\prime \prime}
\end{aligned}
$$




$$
\times \operatorname{tr}\left(T(n) ; S^{*(\tau, \sigma)}\left(2 k, M_{1} \prod_{l \in \Pi} l^{\tilde{\beta}_{l}}\right) \mid R_{\Psi^{\prime}}\right) .
$$

where $\tau:=\left(\tau^{\prime}, \tau^{\prime \prime}\right) \in\{ \pm 1\}^{F} \times\{ \pm 1\}^{D+E}=\{ \pm 1\}^{\Pi}, \sigma \in\{ \pm 1\}^{F(\beta)_{2+}}=$ $\{ \pm 1\}^{\Pi(\tilde{\beta})_{2+}}$, and

$$
S^{*(\tau, \sigma)}\left(2 k, M_{1} \prod_{l \in \Pi} l^{\tilde{\beta}_{l}}\right):=\left\{\begin{array}{l}
f \in S^{*}\left(2 k, M_{1} \prod_{l \in \Pi} l^{\tilde{\beta}_{l}}\right) ; f \mid W_{p}= \\
\tau(p) f \text { for all } p \in \Pi, f\left|R_{p} W_{p}=\sigma(p) f\right| \\
R_{p} \text { for all } l \in \Pi(\tilde{\beta})_{2+}=F(\beta)_{2+}
\end{array}\right\} .
$$

Now we will compare the above formula (2.15) with [U1, (3.10) (2)]. And we will see that the formula (2.15) can be considered as a part of the expression formula $[\mathrm{U} 1,(3.10)(2)]$ of $\operatorname{tr}\left(\tilde{T}\left(n^{2}\right) ; \mathfrak{S}^{\emptyset, \kappa}(k+1 / 2, N, \chi)_{K}\right)$.

We first note that for any parameter $\left(\left(\beta_{l}\right), \Psi^{\prime},\left(\tau^{\prime}, \sigma\right), \tau^{\prime \prime}\right) \in P^{\prime} \times\{ \pm 1\}^{D+E}$, the system $\left(\left(\tilde{\beta}_{l}\right)_{l \in \Pi}, \Psi^{\prime},(\tau, \sigma)\right)$ is contained in the range $P$ of parameters in the expression formula $[\mathrm{U} 1,(3.10)(2)]$ of the $\operatorname{trace} \operatorname{tr}\left(\tilde{T}\left(n^{2}\right) ; \mathfrak{S}^{\emptyset, \kappa}(k+\right.$ $1 / 2, N, \chi)_{K}$. This is easily verified.

We second must study for any $\left(\beta_{l}\right)_{l \in F}, \Psi^{\prime},(\tau, \sigma)=\left(\left(\tau^{\prime}, \sigma\right), \tau^{\prime \prime}\right)$, whether the coefficient of $\operatorname{tr}\left(T(n) ; S^{*(\tau, \sigma)}\left(2 k, M_{1} \prod_{l \in \Pi} l^{\tilde{\beta}_{l}}\right) \mid R_{\Psi^{\prime}}\right)$ is equal to those in the expression formula [U1, (3.10) (2)].

Both coefficients are defined as products of primary components on $p \in \Pi$. We will compare these primary components.

For $p \in F+E$, we can verify the following identities:

$$
\Xi_{p}\left(\left(\tilde{\beta}_{l}\right), \Psi^{\prime},(\tau, \sigma)\right)= \begin{cases}\Xi_{p}^{\prime}\left(\left(\beta_{l}\right), \Psi^{\prime},\left(\tau^{\prime}, \sigma\right)\right), & \text { if } p \in F \\ \Xi_{p}^{\prime \prime}, & \text { if } p \in E\end{cases}
$$

where the left-hand side is the constant with respect to $\mathfrak{S}^{\emptyset, \kappa}(k+1 / 2, N, \chi)_{K}$ determined by $[\mathrm{U} 1,(2.22)]$ and $\tau^{\prime}:=\left.\tau\right|_{F}$.

These identities are easily verified, in case by case, from the following facts.

(1) The 2-primary part of $\chi$ is $\chi_{2}=\eta_{2} \eta_{2}^{\prime}=\left(\frac{\left(\frac{-1}{u}\right)}{)}\right)\left(\frac{\left(\frac{-1}{u^{\prime}}\right)}{}\right), \eta_{2}^{\prime}=\left(\frac{\left(\frac{-1}{u^{\prime}}\right)}{)}\right)$, and also $\eta_{2 M_{1} l_{E}}^{\prime}=\left(\frac{\left(\frac{-1}{u^{\prime}}\right)}{}\right) \chi_{M_{1}} \prod_{p \in E} \chi_{p}$.

(2) For $p \in E+F$, we have $\chi_{p}=\eta_{p}^{\prime}$.

Next we consider the case of $p \in D$. Then if $\tau(p)=-1$, any identity like (2.16) does not hold good. 
However, since any prime $p \in D$ does not occur in the level $M_{1} \prod_{l \in \Pi} l^{\tilde{\beta}_{l}}$, the Atkin-Lehner operator $W_{p}$ is the identity operator 1. Hence if $\tau(p)=$ -1 , the space $S^{*(\tau, \sigma)}\left(2 k, M_{1} \prod_{l \in \Pi} l^{\tilde{\beta}_{l}}\right)$ is always equal to $\{0\}$. Therefore we can neglect such cases and eventually without a loss of validity, we can consider that we also have the same identity as (2.16) in the case of $p \in D$.

Therefore we get the following formula.

Proposition 12. Let notation be the same as above and $n \in \mathbf{Z}_{+}$such that $(n, N)=1$.

$$
\begin{aligned}
\operatorname{tr}\left(\tilde{T}\left(n^{2}\right) ; B^{(\alpha)} \mid e_{\Pi}^{\kappa}\right)= & \sum_{\left(\left(\beta_{l}\right), \Psi^{\prime},\left(\tau^{\prime}, \sigma\right), \tau^{\prime \prime}\right) \in P^{\prime} \times\{ \pm 1\}^{D+E}} \prod_{l \in \Pi} \Xi_{l}\left(\left(\tilde{\beta}_{l}\right), \Psi^{\prime},(\tau, \sigma)\right) \\
& \times \operatorname{tr}\left(T(n) ; S^{*(\tau, \sigma)}\left(2 k, M_{1} \prod_{l \in \Pi} l^{\tilde{\beta}_{l}}\right) \mid R_{\Psi^{\prime}}\right),
\end{aligned}
$$

where each coefficient $\Xi_{l}\left(\left(\tilde{\beta}_{l}\right), \Psi^{\prime},(\tau, \sigma)\right)$ has the value either 0 or 1 which is the coefficient of $\operatorname{tr}\left(T(n) ; S^{*(\tau, \sigma)}\left(2 k, M_{1} \prod_{l \in \Pi} l^{\tilde{\beta}_{l}}\right) \mid R_{\Psi^{\prime}}\right)$ in the expression formula [U1, (3.10) (2)] of the trace of $\tilde{T}\left(n^{2}\right)$ on the space $\mathfrak{S}^{\emptyset, \kappa}(k+$ $1 / 2, N, \chi)_{K}$.

In other words, we can consider the above formula as a part of the expression formula [U1, (3.20) (2)].

For any $\left(\alpha_{l}\right), B^{(\alpha)} \mid e_{\Pi}^{\kappa}$ is a subspace of $\mathfrak{S}^{\emptyset, \kappa}(k+1 / 2, N, \chi)_{K}(\mathrm{cf} .(2.4))$. We define the following subspace of $\mathfrak{S}^{\emptyset, \kappa}(k+1 / 2, N, \chi)_{K}$ :

$$
\hat{\mathfrak{N}}^{\emptyset, \kappa}=\hat{\mathfrak{N}}^{\emptyset, \kappa}(k+1 / 2, N, \chi)_{K}:=\sum_{\left(\alpha_{l}\right) \neq\left(\nu_{l}\right)} B^{(\alpha)} \mid e_{I}^{\kappa} .
$$

Here, $\left(\alpha_{l}\right)$ in the above sum runs over all system of integers such that $0 \leq$ $\alpha_{l} \leq \nu_{l}=\operatorname{ord}_{l}(N)(l \in \Pi)$ and $\left(\alpha_{l}\right)_{l \in \Pi} \neq\left(\nu_{l}\right)_{l \in \Pi}$. We also denote by $\mathfrak{N}^{\emptyset, \kappa}=$ $\mathfrak{N}^{\emptyset, \kappa}(k+1 / 2, N, \chi)_{K}$ the orthogonal complement of $\hat{\mathfrak{N}}^{\emptyset, \kappa}(k+1 / 2, N, \chi)_{K}$ in $\mathfrak{S}^{\emptyset, \kappa}(k+1 / 2, N, \chi)_{K}$.

Since any operators $\tilde{T}\left(n^{2}\right)((n, N)=1)$ fix each subspace $B^{(\alpha)} \mid e_{\Pi}^{\kappa}$ respectively, both $\hat{\mathfrak{N}}^{\emptyset, \kappa}$ and $\mathfrak{N}^{\emptyset, \kappa}$ are stable under the action of $\tilde{T}\left(n^{2}\right)$ $((n, N)=1)$.

We can generalize [U1, Theorem (4.13)] as follows: 
THEOREM 2. Let the notation be the same as above and let $\kappa \in$ $\{ \pm 1\}^{\Pi}$. We suppose that $\operatorname{ord}_{2}(N)=2$. The following assertions hold good.

(1) For all $n \in \mathbf{Z}_{+}$prime to $N$,

$$
\begin{aligned}
& \operatorname{tr}\left(\tilde{T}\left(n^{2}\right) ; \mathfrak{N}^{\emptyset, \kappa}(k+1 / 2, N, \chi)_{K}\right) \\
& =\sum_{\Pi(\nu)_{2}=I+J+K} \sum_{\substack{\tau \in \operatorname{Map}(\Pi,\{ \pm 1\}) \\
\sigma \in \operatorname{Map}(\Pi-(I+J),\{ \pm 1\})}} \Xi\left(\left(\nu(I, J)_{l}\right), I+J,(\tau, \sigma)\right) \\
& \quad \times \operatorname{tr}\left(T(n) ; S^{*(\tau, \sigma)}\left(2 k, M_{1} \prod_{l \in J} l \prod_{l \in \Pi-(I+J)} l^{\nu_{l}}\right) \mid R_{I+J}\right),
\end{aligned}
$$

where $\Pi(\nu)_{2}:=\left\{l \in \Pi \mid \nu_{l}=2\right\}, \sum_{\Pi(\nu)_{2}=I+J+K}$ is the sum extended over all partitions such that $\Pi(\nu)_{2}=I+J+K, \nu(I, J)_{l}$ is a constant which has a value 0,1 , or $\nu_{l}\left(:=\operatorname{ord}_{l}(N)\right)$ according to $l \in I, J$, or $\Pi-(I+J)$, $\Xi\left(\left(\nu(I, J)_{l}\right), I+J,(\tau, \sigma)\right)$ are the constants determined by [U1, (2.22)].

(2) Let $\underline{\mathcal{B}}$ be an orthogonal basis of $\mathfrak{S}^{\emptyset, \kappa}(k+1 / 2, N, \chi)_{K}$ which is stated in $[\mathrm{U} 1,(3.11)(1)]$. Let $\underline{\underline{\mathcal{B}_{0}}}\left(\right.$ resp. $\left.\underline{\underline{\mathcal{B}_{1}}}\right)$ be the set of all $f \in \underline{\underline{\mathcal{B}}}$ which correspond to primitive forms in $\overline{\overline{S^{0}}}(2 k, M)\left(\right.$ resp. in $S^{0}\left(2 k, M^{\prime}\right), 0<M^{\prime} \mid M$, and $\left.M^{\prime}<M\right)$ in the sense of $\left[\mathrm{U} 1\right.$, Theorem (3.11) (1)]. Then $\underline{\underline{\mathcal{B}_{0}}}$ (resp. $\underline{\underline{\mathcal{B}_{1}}}$ ) generates the space $\mathfrak{N}^{\emptyset, \kappa}(k+1 / 2, N, \chi)_{K}\left(\right.$ resp. $\left.\hat{\mathfrak{N}}^{\emptyset, \kappa}(k+1 / 2, N, \chi)_{K}\right)$.

(3) Let $f$ be any element of $\underline{\mathcal{B}_{0}}$ and $\lambda_{p}$ the eigenvalue of $f$ with respect to $\tilde{T}_{k+1 / 2, N, \chi}\left(p^{2}\right)$ ( $p$ : prime, $\left.p \nmid \bar{M}\right)$ resp. $U\left(p^{2}\right)(p$ : prime, $p \mid M)$. Then the primitive form $F$ which corresponds to $f$ in the sense of [U1, Theorem (3.11) (1)] satisfies the condition $F \mid T(p)=\lambda_{p} F$ resp. $F \mid U(p)=\lambda_{p} F$ for all primes with $p \nmid M$ resp. $p \mid M$.

Proof. Let $\underline{\mathcal{B}}$ be the same C-basis of $\mathfrak{S}^{\emptyset, \kappa}(k+1 / 2, N, \chi)_{K}$ as in the above statement (2) and $P$ the same set of parameters as in the expression formula [U1, (3.10) (2)].

For any $\left(\alpha_{l}\right)$, the space $B^{(\alpha)} \mid e_{\Pi}^{\kappa}$ is a stable subspace of $\mathfrak{S}^{\emptyset, \kappa}(k+$ $1 / 2, N, \chi)_{K}$ under the action of all operators $\tilde{T}\left(n^{2}\right)((n, N)=1)$. Using the Strong Multiplicity One theorem [U1, (3.11) (2)] on $\mathfrak{S}^{\emptyset, \kappa}(k+1 / 2, N, \chi)_{K}$, $B^{(\alpha)} \mid e_{\Pi}^{\kappa}$ is generated by the set $\underline{\underline{\mathcal{B}}} \cap\left(B^{(\alpha)} \mid e_{\Pi}^{\kappa}\right)$. Similarly, we can see that the spaces $\hat{\mathfrak{N}}^{\emptyset, \kappa}$ and $\mathfrak{N}^{\emptyset, \kappa}$ are generated by $\underline{\underline{\mathcal{B}}} \cap \hat{\mathfrak{N}}^{\emptyset, \kappa}=\cup_{\left(\alpha_{l}\right) \neq\left(\nu_{l}\right)}\left(B^{(\alpha)} \mid e_{\Pi}^{\kappa}\right) \cap$ $\underline{\underline{\mathcal{B}}}$ and $\mathfrak{N}^{\emptyset, \kappa} \cap \underline{\underline{\mathcal{B}}}$ respectively. And also $\underline{\underline{\mathcal{B}}}=\left(\underline{\underline{\mathcal{B}}} \cap \mathfrak{N}^{\emptyset, \kappa}\right)+\left(\underline{\underline{\mathcal{B}}} \cap \hat{\mathfrak{N}}^{\emptyset, \kappa}\right)$. For 
any $\left(\left(\rho_{l}\right), \Psi,(\tau, \sigma)\right) \in P$, we denote by $\underline{\underline{\mathcal{B}}}_{\left(\left(\rho_{l}\right), \Psi,(\tau, \sigma)\right)}$ the subset of $\underline{\underline{\mathcal{B}}}_{\text {which }}$ corresponds to the subspace $S^{*(\tau, \sigma)}\left(2 k, M_{1} \prod_{l \in \Pi} l^{\rho_{l}}\right) \mid R_{\Psi}$ in the sense of [U1, (3.11) (1)].

Then from Proposition 12, there exist only two possible cases: either $\underline{\underline{\mathcal{B}}}_{\left(\left(\rho_{l}\right), \Psi,(\tau, \sigma)\right)} \subseteq B^{(\alpha)} \mid e_{\Pi}^{\kappa}$, or $\underline{\underline{\mathcal{B}}}_{\left(\left(\rho_{l}\right), \Psi,(\tau, \sigma)\right)} \cap B^{(\alpha)} \mid e_{\Pi}^{\kappa}=\emptyset$; and whether the former case is or not depends only on the parameter $\left(\left(\rho_{l}\right), \Psi,(\tau, \sigma)\right)$.

We define the subsets $P^{*}$ and $P^{* *}$ of $P$ by:

$$
P^{*}:=\left\{\begin{array}{l}
\left(\left(\rho_{l}\right), \Psi,(\tau, \sigma)\right) \in P ;\left(\left(\rho_{l}\right), \Psi,(\tau, \sigma)\right) \text { does not satisfy (at least) } \\
\text { one of the following three conditions: }(\mathrm{i}) \rho_{l}=\nu_{l} \text { on } \Pi(\rho)_{2+} ; \\
(\mathrm{ii}) \Psi=\Pi(\rho)_{0}+\Pi(\rho)_{1} ;\left(\text { iii) } \nu_{l}=2 \text { on } \Pi(\rho)_{0}+\Pi(\rho)_{1}\right.
\end{array}\right\}
$$

and $P^{* *}:=P-P^{*}$.

Take a system $\left(\alpha_{l}\right)_{l \in \Pi}$ and a parameter $\left(\left(\rho_{l}\right), \Psi,(\tau, \sigma)\right) \in P$ such that $\left(\alpha_{l}\right) \neq\left(\nu_{l}\right)$ and $\underline{\underline{\mathcal{B}}}_{\left(\left(\rho_{l}\right), \Psi,(\tau, \sigma)\right)} \subseteq B^{(\alpha)} \mid e_{\Pi}^{\kappa}$. Then $\left(\left(\rho_{l}\right), \Psi,(\tau, \sigma)\right) \in P^{*}$. In fact, suppose that $\left(\left(\rho_{l}\right), \Psi,(\tau, \sigma)\right)$ satisfies the above condition (i) and (ii). We get from Proposition 12 that $\rho_{l} \leq \alpha_{l}$ for all $l \in \Pi$ and $\Psi \subseteq \Pi(\alpha)_{2+}$. Hence $\Pi(\rho)_{2+} \subseteq \Pi(\alpha)_{2+}$ and by using the condition (i), $\left(\rho_{l}=\right) \alpha_{l}=\nu_{l}$ for all $l \in \Pi(\rho)_{2+}$.

From the condition (ii), $\Pi(\alpha)_{2+} \supseteq \Psi=\Pi-\Pi(\rho)_{2+} \supseteq \Pi-\Pi(\alpha)_{2+}$. This means $\Pi=\Pi(\alpha)_{2+}$.

Since $\left(\alpha_{l}\right) \neq\left(\nu_{l}\right)$, there exists $l \in \Pi$ such that $\alpha_{l}<\nu_{l}$. From the above, then $l \in \Pi-\Pi(\rho)_{2+}\left(\subseteq \Pi(\alpha)_{2+}\right)$ and $2 \leq \alpha_{l}<\nu_{l}$. Hence the condition (iii) is not satisfied.

The contrary is also true. Take any $\left(\left(\rho_{l}\right), \Psi,(\tau, \sigma)\right) \in P^{*}$. Put

$$
\alpha_{l}:= \begin{cases}\rho_{l}, & \text { if } l \in \Pi(\rho)_{2+}, \\ 2, & \text { if } l \in \Psi, \\ 1, & \text { if } l \in \Pi(\rho)_{1}-\Psi \\ 0, & \text { if } l \in \Pi(\rho)_{0}-\Psi\end{cases}
$$

Since $\left(\left(\rho_{l}\right), \Psi,(\tau, \sigma)\right)$ does not satisfy one of the conditions (i)-(iii), we have $\left(\alpha_{l}\right) \neq\left(\nu_{l}\right)$. Next we put

$$
\beta_{l}:= \begin{cases}\rho_{l}, & \text { if } l \in \Pi(\rho)_{2+}, \\ 1, & \text { if } l \in \Psi \cap \Pi(\rho)_{1}, \\ 0, & \text { if } l \in \Psi \cap \Pi(\rho)_{0} .\end{cases}
$$

We also define the system of integers $\left(\tilde{\beta}_{l}\right)_{l \in \Pi}$ by the above $\left(\beta_{l}\right)$ in the same manner as in the paragraph after the formula (2.14). Then we have 
$\tilde{\beta}_{l}=\rho_{l}$ for all $l \in \Pi$. Moreover observing that $\Psi \subseteq \Pi(\rho)_{0}+\Pi(\rho)_{1} \subseteq$ $\left(\Pi(\alpha)_{2+}\right)(\beta)_{0}+\left(\Pi(\alpha)_{2+}\right)(\beta)_{1}$ and $\left(\Pi(\alpha)_{2+}\right)(\beta)_{2+}=\Pi(\rho)_{2+}$, we can see that $\left(\left(\beta_{l}\right)_{l \in \Pi(\alpha)_{2+}}, \Psi,(\tau, \sigma)\right)$ occurs in the parameter set $P^{\prime} \times\{ \pm 1\}^{\Pi(\alpha)_{1}+\Pi(\alpha)_{0}}$

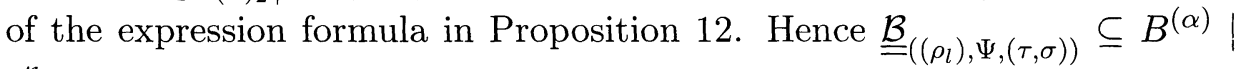
$e_{\Pi}^{\kappa}$.

Thus, we see that $\hat{\mathfrak{N}}^{\emptyset, \kappa}$ is generated by the set $\bigcup_{\left(\left(\rho_{l}\right), \Psi,(\tau, \sigma)\right) \in P^{*}} \underline{\underline{\mathcal{B}}}_{\left(\left(\rho_{l}\right), \Psi,(\tau, \sigma)\right)}$ and so $\cup_{\left(\left(\rho_{l}\right), \Psi,(\tau, \sigma)\right) \in P^{* *}} \underline{\underline{B}}_{\left(\left(\rho_{l}\right), \Psi,(\tau, \sigma)\right)}$ generates $\mathfrak{N}^{\emptyset, \kappa}$. The assertion (1) is easily deduced from this result.

We will prove the assertion $(2)$. For any $\left(\left(\rho_{l}\right), \Psi,(\tau, \sigma)\right) \in P$, we have from the definition: $S^{*(\tau, \sigma)}\left(2 k, M_{1} \prod_{l \in \Pi} l^{\rho_{l}}\right) \mid R_{\Psi} \subseteq S^{0}\left(2 k, M_{1} \prod_{l \in \Pi-\Psi} l^{\rho_{l}}\right.$ $\left.\prod_{l \in \Psi} l^{2}\right)$. Hence the assertion (2) follows from the fact:

$$
\left(\left(\rho_{l}\right), \Psi,(\tau, \sigma)\right) \in P^{* *} \Longleftrightarrow \prod_{l \in \Pi-\Psi} l^{\rho_{l}} \prod_{l \in \Psi} l^{2}=M_{2+} .
$$

The assertion (3) can be proved by the same method as in the [U1, Theorem $(3.11)(1)]$.

\section{$\S 3$. Another simpler definition of newforms for Kohnen spaces}

In this section, we give another simpler definition of newforms for Kohnen spaces than the previous one.

We keep to the notations in the previous sections and the paper [U1]. See those for the details of definitions and notations.

We note that we consider only Kohnen spaces in this section.

In this paper and the previous paper [U1], we define several subspaces and give several decompositions. Combining those decompositions, we obtain the following:

$$
\begin{aligned}
& \left\{\begin{array}{ll}
S:=S(k+1 / 2, N, \chi)_{K} & \text { if } k \geq 2 \\
V:=V(N ; \chi)_{K} & \text { if } k=1
\end{array}\right\} \\
& =\left(\bigoplus_{\kappa \in\{ \pm 1\}^{\Pi}} \mathfrak{N}^{\emptyset, \kappa}(k+1 / 2, N, \chi)_{K}\right) \\
& \oplus\left(\bigoplus_{\kappa \in\{ \pm 1\}^{I I}} \hat{\mathfrak{N}}^{\emptyset, \kappa}(k+1 / 2, N, \chi)_{K}\right)
\end{aligned}
$$




$$
\begin{aligned}
& \oplus\left(\bigoplus_{\kappa \in\{ \pm 1\}^{\Pi}} \bigoplus_{\begin{array}{c}
0<e, d, d \neq M_{1} \\
e d \mid M_{1}
\end{array}} \mathfrak{S}^{\emptyset, \kappa}\left(k+1 / 2,4 d M_{2+}, \chi\right)_{K} \mid U\left(e^{2}\right)\right) \\
& \oplus\left\{\begin{array}{ll}
\operatorname{Ker}\left(R_{\Pi}, S\right) & \text { if } k \geq 2 \\
\operatorname{Ker}\left(R_{\Pi}, V\right) & \text { if } k=1
\end{array}\right\} .
\end{aligned}
$$

This formula follows from [U1, (1.5), (1.7), (3.10) (2)] and (2.17) in this paper.

DEFinition. We put the following notation:

(3.2) $\mathfrak{N}(k+1 / 2, N, \chi)_{K}$

$$
:=\bigoplus_{\kappa \in\{ \pm 1\}^{\Pi}} \mathfrak{N}^{\emptyset, \kappa}(k+1 / 2, N, \chi)_{K},
$$

$$
\begin{aligned}
\mathfrak{O}(k+1 / 2, N, \chi)_{K} & \left(\bigoplus_{\kappa \in\{ \pm 1\}^{\Pi}} \hat{\mathfrak{N}}^{\emptyset, \kappa}(k+1 / 2, N, \chi)_{K}\right) \\
& \oplus\left(\bigoplus_{\kappa \in\{ \pm 1\}^{\Pi}} \bigoplus_{\substack{0<e, d, d \neq M_{1} \\
\text { ed|M }}} \mathfrak{S}^{\emptyset, \kappa}\left(k+1 / 2,4 d M_{2+}, \chi\right)_{K} \mid U\left(e^{2}\right)\right) \\
& \oplus\left\{\begin{array}{ll}
\operatorname{Ker}\left(R_{\Pi}, S\right) & \text { if } k \geq 2 \\
\operatorname{Ker}\left(R_{I I}, V\right) & \text { if } k=1
\end{array}\right\} .
\end{aligned}
$$

Then we can rewrite the formula (3.1) as follows.

$$
\begin{aligned}
\left\{\begin{array}{rr}
S:=S(k+1 / 2, N, \chi)_{K}, & \text { if } k \geq 2 \\
V:=V(N ; \chi)_{K}, & \text { if } k=1
\end{array}\right\} \\
=\mathfrak{N}(k+1 / 2, N, \chi)_{K} \oplus \mathfrak{O}(k+1 / 2, N, \chi)_{K} .
\end{aligned}
$$

Proposition 13.

$$
\begin{aligned}
\operatorname{Ker}\left(R_{\Pi}, S\right) & =\sum_{l \in \Pi} S\left(k+1 / 2, N / l, \chi\left(\frac{l}{-}\right)\right)_{K} \mid \tilde{\delta}_{l} . \\
\operatorname{Ker}\left(R_{\Pi}, V\right) & =\sum_{l \in \Pi} V\left(N / l ; \chi\left(\frac{l}{-}\right)\right)_{K} \mid \tilde{\delta}_{l} .
\end{aligned}
$$


Proof. By using $[\mathrm{U} 1,(1.9)]$, the subspaces of the right-hand sides are contained in $S, V$ respectively. Since $\left(f \mid \tilde{\delta}_{l}\right)(z)=l^{k / 2+1 / 4} f(l z)$, those subspaces are contained in the subspaces of left-hand sides.

We will prove the contrary. Let $I$ be any non-empty subset of $\Pi$.

From [U1, (1.5) (3)], it is sufficient to prove that $S^{I}, V^{I}$ are contained in the subspaces of right-hand sides respectively.

From the formula after $[\mathrm{U} 1,(1.4)]$, we have

$$
\begin{aligned}
S^{I} & =\left\{\sum_{n \geq 1} b(n) \mathbf{e}(n z) \in S ; b(n)=0 \text { for all } n \notin L_{I}\right\}, \\
V^{I} & =\left\{\sum_{n \geq 1} b(n) \mathbf{e}(n z) \in V ; b(n)=0 \text { for all } n \notin L_{I}\right\},
\end{aligned}
$$

where $L^{I}:=\left\{a \in \mathbf{Z} ;\left(a, l_{\Pi}\right)=l_{I}\right\}\left(\subseteq \mathbf{Z} l_{I}\right)$.

Take a prime $l \in I$ and any element $f=\sum_{n=1}^{\infty} b(n) \mathbf{e}(n z)$ in $S^{I}$ or $V^{I}$. Then from the above formula, $b(n) \neq 0 \Rightarrow n \in L_{I} \subseteq \mathbf{Z l}$. Hence there exists a function $g(z)$ on $\mathfrak{H}$ such that $f(z)=g(l z)$. It follows from [U1, (1.11)] that $g \in S\left(k+1 / 2, N / l, \chi\left(l^{-}\right)\right)_{K}$ if $f \in S^{I}$, or $g \in V\left(N / l ; \chi\left(l^{-}\right)\right)_{K}$ if $f \in V^{I}$. This proves the contrary inclusion.

Definition. For any primitive form $F$ of weight $2 k$, we denote by $\left\{\lambda_{F}(n) ; n \in \mathbf{N}\right\}$ the system of eigenvalues on the Hecke operators $\{T(n) ; n \in$ $\mathrm{N}\}$. Then we define two subspaces of $S(k+1 / 2, N, \chi)_{K}$ as follows:

$$
\begin{aligned}
S(k+1 / 2, N, \chi ; F)_{K} & :=\left\{\begin{array}{l}
f \in S(k+1 / 2, N, \chi)_{K} ; f \mid \tilde{T}\left(n^{2}\right)=\lambda_{F}(n) f \\
\text { for all } n \in \mathbf{Z}_{+} \text {such that }(n, N)=1
\end{array}\right\} . \\
V(N, \chi ; F)_{K} & :=\left\{\begin{array}{l}
f \in V(N ; \chi)_{K} ; f \mid \tilde{T}\left(n^{2}\right)=\lambda_{F}(n) f \text { for all } \\
n \in \mathbf{Z}_{+} \text {such that }(n, N)=1
\end{array}\right\} .
\end{aligned}
$$

These are considered as the eigen subspaces corresponding to the primitive form $F$.

Remark. We can prove that $V(N, \chi ; F)_{K}=S(3 / 2, N, \chi ; F)_{K}$ for any primitive (cusp) form $F$.

Under the above notation, we get the following theorem. 
THEOREM 3. (1) For $k \geq 2$, the following decompositions hold good.

$$
\begin{aligned}
S(k+1 / 2, N, \chi)_{K} & =\bigoplus_{F:(* 1)} S(k+1 / 2, N, \chi ; F)_{K}, \\
\mathfrak{N}(k+1 / 2, N, \chi)_{K} & =\bigoplus_{F:(* 2)} S(k+1 / 2, N, \chi ; F)_{K}, \\
\mathfrak{O}(k+1 / 2, N, \chi)_{K} & =\bigoplus_{F:(* 3)} S(k+1 / 2, N, \chi ; F)_{K} .
\end{aligned}
$$

(2) For $k=1$, the following decompositions hold good.

$$
\begin{aligned}
V(N ; \chi)_{K} & =\bigoplus_{F:(* 1)} V(N, \chi ; F)_{K}, \\
\mathfrak{N}(3 / 2, N, \chi)_{K} & =\bigoplus_{F:(* 2)} V(N, \chi ; F)_{K} \\
\mathfrak{O}(3 / 2, N, \chi)_{K} & =\bigoplus_{F:(* 3)} V(N, \chi ; F)_{K} .
\end{aligned}
$$

Here $(* 1)-(* 3)$ are the following conditions on primitive forms $F$ of weight $2 k$ :

(*1) the conductor of $F$ is a divisor of $M$.

(*2) the conductor of $F$ is $M$.

(*3) the conductor of $F$ is a divisor of $M$ and less than $M$.

Proof. From Theorem $2(2)$, the subspace $\mathfrak{N}^{\emptyset, \kappa}(k+1 / 2, N, \chi)_{K}$ has an orthogonal C-basis consisting of common eigenforms for all Hecke operators $\tilde{T}\left(n^{2}\right)\left(n \in \mathbf{Z}_{+},(n, N)=1\right)$. Moreover the system of eigenvalues of any element of such basis corresponds to a primitive form of weight $2 k$ and of conductor $M$.

Similarly, from Theorem 2 (2) and [U1, (3.10) (2) and (3.5)], the subspaces $\hat{\mathfrak{N}}^{\emptyset, \kappa}(k+1 / 2, N, \chi)_{K}$ and $\mathfrak{S}^{\emptyset, \kappa}\left(k+1 / 2,4 d M_{2+}, \chi\right)_{K} \mid U\left(e^{2}\right)$ have orthogonal $\mathbf{C}$-basis consisting of common eigenforms for all $\tilde{T}\left(n^{2}\right)\left(n \in \mathbf{Z}_{+}\right.$, $(n, N)=1)$. Moreover the system of eigenvalues of any element of such basis corresponds to a primitive form of weight $2 k$ and of a conductor which divides $M$ and is less than $M$.

We will prove a similar result for the subspaces $\operatorname{Ker}\left(R_{\Pi}, S\right)$ and $\operatorname{Ker}\left(R_{\Pi}\right.$, $V)$, i.e., these subspaces have orthogonal $\mathbf{C}$-basis consisting of common 
eigenforms for all $\tilde{T}\left(n^{2}\right)\left(n \in \mathbf{Z}_{+},(n, N)=1\right)$ and moreover the system of eigenvalues of any element of such basis corresponds to a primitive form of weight $2 k$ and of a conductor which divides $M$ and is less than $M$.

We use an induction on $M_{2+}$.

If $M_{2+}=1$, then $\Pi=\emptyset$ and $R_{\Pi}=\mathbf{1}$. Hence, $\operatorname{Ker}\left(R_{\Pi}, S\right)=\operatorname{Ker}\left(R_{\Pi}, V\right)$ $=\{0\}$ and the statement holds good.

Let $M_{2+}>1$ and assume that the above claim holds good if the subspace has a smaller " $M_{2+}$ "-part than $M_{2+}$.

We consider the spaces $S\left(k+1 / 2, N / l, \chi\left(l^{-}\right)\right)_{K}$ and $V(N / l ; \chi(\stackrel{l}{)}))_{K}$ for any $l \in \Pi$. From the assumption of the induction and [U1, (1.8)], these subspaces have orthogonal $\mathbf{C}$-basis consisting of common eigenforms for all $\tilde{T}\left(n^{2}\right)\left(n \in \mathbf{Z}_{+},(n, N)=1\right)$ and moreover the system of eigenvalues of any element of such basis corresponds to a primitive form of weight $2 k$ and of conductor dividing $M / l$.

Combining this and Proposition 13, the claim for $\operatorname{Ker}\left(R_{\Pi}, S\right)$ and $\operatorname{Ker}\left(R_{\Pi}, V\right)$ follows.

From (3.1)-(3.3) and the Strong Multiplicity One theorem of weight $2 k$, the first assertion follows. The second and third assertions are easily seen by using the above results.

Our next purpose is to rewrite the space of "oldform" $\mathfrak{O}(k+1 / 2, N, \chi)_{K}$.

Proposition 14. We have the following formulae.

[The case of $k \geq 2$ ]

$$
\begin{aligned}
\mathfrak{O}(k+1 / 2, N, \chi)_{K} & =\sum_{\substack{0<B \mid M \\
B \neq M}} \sum_{\substack{0<A \mid(M / B) \\
\xi(\underline{A})=\chi}} S(k+1 / 2,4 B, \xi)_{K} \mid \tilde{\delta}_{A} \\
& +\sum_{\substack{0<B \mid M \\
B \neq M}} \sum_{\substack{0<A \mid(M / B)^{2} \\
\xi(\underline{A})=\chi}} \sum_{\substack{0 \leq e_{l} \leq 2 \\
(l \in \Pi)}} S(k+1 / 2,4 B, \xi)_{K} \mid U(A) \prod_{l \in \Pi} R_{l} e_{l} .
\end{aligned}
$$

[The case of $k=1$ ]

$$
\begin{aligned}
\mathfrak{O}(3 / 2, N, \chi)_{K}= & \sum_{\substack{0<B \mid M \\
B \neq M}} \sum_{\substack{0<A \mid(M / B) \\
\xi(\underline{A})=\chi}} V(4 B ; \xi)_{K} \mid \tilde{\delta}_{A} \\
& +\sum_{\substack{0<B \mid M \\
B \neq M}} \sum_{\substack{0<A \mid(M / B)^{2} \\
\xi(\underline{A})=\chi}} \sum_{\substack{0 \leq e_{l} \leq 2 \\
(l \in \Pi)}} V(4 B ; \xi)_{K} \mid U(A) \prod_{l \in \Pi} R_{l}^{e_{l}} .
\end{aligned}
$$


Here, $\xi$ runs over all (even quadratic) Dirichlet characters defined modulo $4 B$ such that $\xi(\stackrel{A}{)})=\chi$.

Remark. We know the relation of the twisting operator: $R_{l}{ }^{3}=R_{l}$ for all $l \in \Pi$. Hence we can replace the range $0 \leq e_{l} \leq 2$ with $0 \leq e_{l} \in \mathbf{Z}$.

Proof. From $[\mathrm{U} 1,(1.9),(1.22),(1.23)]$, we easily see that the right-hand sides of the statements are subspaces of $S(k+1 / 2, N, \chi)_{K}$ resp. $V(N ; \chi)_{K}$.

The subspaces $S(k+1 / 2,4 B, \xi)_{K}$ and $V(4 B ; \xi)_{K}$ have orthogonal Cbasis consisting of common eigenforms on hermitian operator $\tilde{T}\left(n^{2}\right)(n \in$ $\left.\mathbf{Z}_{+},(n, 4 B)=1\right)$, moreover the systems of eigenvalues of such eigenforms correspond to primitive forms of weight $2 k$ and of conductors dividing $B$. (cf. [U1, (3.10)]).

The operators $\tilde{T}\left(n^{2}\right)\left(n \in \mathbf{Z}_{+},(n, N)=1\right)$ commute with the operators $\tilde{\delta}_{A}, U(A)$, and $R_{l}(l \in \Pi)$ (cf. [U1, (1.8), (1.20)] and [U3, (1.7)]). Hence all subspaces in the right-hand sides of the statements have generators which are common eigenforms on $\tilde{T}\left(n^{2}\right)\left(n \in \mathbf{Z}_{+},(n, N)=1\right)$. Moreover those systems of eigenvalues correspond to primitive forms of weight $2 k$ and of a conductor which divides $M$ and is less than $M$, because $B \mid M$ and $B \neq M$.

Combining this with Theorem 3 , we see that $\mathfrak{O}(k+1 / 2, N, \chi)_{K}$ contains the right-hand side.

Next we will prove the contrary inclusion.

We have an explicit expression formula (3.3) of $\mathfrak{O}(k+1 / 2, N, \chi)_{K}$. We will check each direct summand of the formula (3.3).

It follows from Proposition 13 that $\operatorname{Ker}\left(R_{\Pi}, S\right)$ and $\operatorname{Ker}\left(R_{\Pi}, V\right)$ occur in the first term of the right-hand side, respectively.

Next we consider the part of the subspaces $\mathfrak{S}^{\emptyset, \kappa}\left(k+1 / 2,4 d M_{2+}, \chi\right)_{K}$ $U\left(e^{2}\right)=\mathfrak{S}^{\emptyset, \kappa^{\prime}}\left(k+1 / 2,4 d M_{2+}, \chi^{2+}\right)_{K} \mid U\left(m_{1} e^{2}\right)\left(0<e, d, d \neq M_{1}, e d \mid\right.$ $\left.M_{1}\right)$, where see $[\mathrm{U} 1,(3.1)]$ for the definition of $m_{1}$ and $\chi^{2+}$. We only remark $\kappa^{\prime}:=\kappa \cdot\left(\underline{m_{1}}\right), 0<m_{1} \mid M_{1}$, and $\chi^{2+}\left(\underline{m_{1}}\right)=\chi$.

This subspace is contained in the space $S\left(k+1 / 2,4 d M_{2+}, \chi^{2+}\right)_{K}$ $U\left(m_{1} e^{2}\right)$ (when $\left.k \geq 2\right)$, resp. $V\left(4 d M_{2+} ; \chi^{2+}\right)_{K} \mid U\left(m_{1} e^{2}\right)$ (when $\left.k=1\right)$.

We decompose $m_{1}=m_{2} \cdot m_{3}$ with $m_{2}=\left(m_{1}, d\right)$.

Then the character $\chi^{2+}\left(\frac{m_{2}}{2}\right)$ can be defined modulo $4 d M_{2+}$. From [U1, (1.28)], we have $S\left(k+1 / 2,4 d M_{2+}, \chi^{2+}\right)_{K} \mid U\left(m_{2}\right)=S\left(k+1 / 2,4 d M_{2+}\right.$, $\left.\chi^{2+}\left(\underline{m_{2}}\right)\right)_{K}$ and $V\left(4 d M_{2+} ; \chi^{2+}\right)_{K} \mid U\left(m_{2}\right)=V\left(4 d M_{2+} ; \chi^{2+}\left(\underline{m_{2}}\right)\right)_{K}$.

From this we may assume that $\left(m_{1}, d\right)=1$. 
We decompose that $m_{1}=m_{4} \cdot m_{5}$ with $m_{4}=\left(m_{1}, e\right)$.

We first consider the case of $m_{4}=1$. In this case, we set $B=d M_{2+}$.

Since $\left(m_{1}, e d\right)=(e, d)=1, m_{1} e^{2} \mid\left(M_{1} / d\right)^{2}=(M / B)^{2}$ and so the subspaces $S\left(k+1 / 2,4 d M_{2+}, \chi^{2+}\right)_{K} \mid U\left(m_{1} e^{2}\right)$ (when $\left.k \geq 2\right)$ and $V\left(4 d M_{2+}\right.$; $\left.\chi^{2+}\right)_{K} \mid U\left(m_{1} e^{2}\right)$ (when $k=1$ ) occur in the second term of the right hand side.

Next we consider the case of $m_{4}>1$. Take a prime divisor $p$ of $m_{4}$. Then $m_{1} e^{2}=\left(m_{1} / p\right)(e / p)^{2} \times p^{3}$.

For $k \geq 2$, we have

$$
\begin{aligned}
S(k+ & \left.1 / 2,4 d M_{2+}, \chi^{2+}\right)_{K} \mid U\left(m_{1} e^{2}\right) \\
= & S\left(k+1 / 2,4 d M_{2+}, \chi^{2+}\right)_{K} \mid U\left(\left(m_{1} / p\right)(e / p)^{2}\right) U\left(p^{3}\right) \\
\subseteq & S\left(k+1 / 2,4 M / p, \chi^{2+}\left(\frac{m_{1} / p}{}\right)\right)_{K} \mid U\left(p^{3}\right) \\
\subseteq & S\left(k+1 / 2,4 M / p, \chi^{2+}\left(\frac{m_{1} / p}{}\right)\right)_{K} \mid \tilde{\delta}_{p} \\
& +S\left(k+1 / 2,4 M / p, \chi^{2+}\left(\frac{m_{1} / p}{)^{2}}\right)_{K} \mid U(p) .\right.
\end{aligned}
$$

Here, we use the formula (A.4) at the last inclusion.

For $k=1$, we can see in the same way,

$$
\begin{aligned}
V\left(4 d M_{2+} ; \chi^{2+}\right)_{K} \mid U\left(m_{1} e^{2}\right) \subseteq & V\left(4 M / p ; \chi^{2+}\left(\frac{m_{1} / p}{)}\right)\right)_{K} \mid \tilde{\delta}_{p} \\
& +V\left(4 M / p ; \chi^{2+}\left(\frac{m_{1} / p}{)^{2}}\right)\right)_{K} \mid U(p) .
\end{aligned}
$$

Here, we use that Hecke operator $\tilde{T}\left(p^{2}\right)$ fixes $V\left(4 M / p ; \chi^{2+}\left(\frac{m_{1} / p}{}\right)\right)_{K}$.

We set $B=M / p, A=p$ and so $A=p \mid(M / B)=p$. This means that $S\left(k+1 / 2,4 d M_{2+}, \chi^{2+}\right)_{K} \mid U\left(m_{1} e^{2}\right)$ (when $\left.k \geq 2\right)$ and $V\left(4 d M_{2+} ; \chi^{2+}\right)_{K} \mid$ $U\left(m_{1} e^{2}\right)$ (when $k=1$ ) are contained in the right-hand side.

We finally consider the part of $\hat{\mathfrak{N}}^{\emptyset, \kappa}(k+1 / 2, N, \chi)_{K}$. For all $\left(\alpha_{l}\right)_{l \in \Pi} \neq$

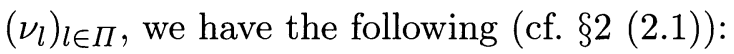

$$
\begin{aligned}
B^{(\alpha)} & :=\bigoplus_{0<a \mid l_{D}} \mathfrak{S}^{\emptyset,\left.\kappa\right|_{F}}(k+1 / 2, \tilde{N}(\alpha), \chi)_{K} \mid U\left(a^{2}\right) \\
& :=\bigoplus_{0<a \mid l_{D}} \mathfrak{S}^{\emptyset,\left.\kappa^{\prime}\right|_{F}}\left(k+1 / 2, \tilde{N}(\alpha), \eta^{\prime}\right)_{K} \mid U\left(u a^{2}\right),
\end{aligned}
$$


where $D:=\Pi(\alpha)_{0}, F:=\Pi(\alpha)_{2+}$, and see $\S 2$ for the other notation.

It follows from the above that $B^{(\alpha)}$ occurs in the second term of the right-hand side. Here, we note that $B=M_{1} M_{2+}^{(\alpha)}$ and so $l_{D}{ }^{2} \mid(M / B)$. Hence $A=u a^{2}\left|l_{D}^{3}\right|(M / B)^{2}$.

Since $e_{\Pi}^{\kappa}=\prod_{l \in \Pi} \frac{1}{2}\left(R_{l}^{2}+\kappa(l) R_{l}\right)$ and $\hat{\mathfrak{N}}^{\emptyset, \kappa}(k+1 / 2, N, \chi)_{K}=\sum_{\left(\alpha_{l}\right) \neq\left(\nu_{l}\right)}$ $B^{(\alpha)} \mid e_{\Pi}^{\kappa}$, the subspace $\hat{\mathfrak{N}}^{\emptyset, \kappa}(k+1 / 2, N, \chi)_{K}$ is contained in the second term of the right-hand side.

From the above, we can give the following simple reformulation of results of $\S 2$ and $\S 3$.

THEOREM 4. (Final formulation of newform for Kohnen space) Let $k$ be a positive integer and $M$ an odd positive integer. Let $\chi$ be an even character modulo $N:=4 M$ with $\chi^{2}=1$. We denote the $p$-adic additive valuation for any integer $m$ by $\operatorname{ord}_{p}(m)$. We decompose $M$ as follows:

$$
M=M_{1} M_{2+}, \quad M_{1}:=\prod_{\substack{\operatorname{ord} p(M)=1 \\ p \mid M}} p, \quad M_{2+}:=\prod_{\substack{\operatorname{ord} p(M) \geq 2 \\ p \mid M}} p^{\operatorname{ord}_{p}(M)} .
$$

Denote the set of all prime divisors of $M_{2+}$ by $\Pi$.

Define the space of oldform $\mathfrak{O}(k+1 / 2, N, \chi)_{K}$ as follows.

[The case of $k \geq 2$ ]

$$
\begin{aligned}
\mathfrak{O}(k+1 / 2, N, \chi)_{K} & \sum_{\substack{0<B \mid M \\
B \neq M}} \sum_{\substack{0<A \mid(M / B) \\
\xi(\underline{A})=\chi}} S(k+1 / 2,4 B, \xi)_{K} \mid \tilde{\delta}_{A} \\
& +\sum_{\substack{0<B \mid M \\
B \neq M}} \sum_{\substack{0<A \mid(M / B)^{2} \\
\xi(\underline{A})=\chi}} \sum_{\substack{0 \leq e_{l} \leq 2 \\
(l \in \Pi)}} S(k+1 / 2,4 B, \xi)_{K} \mid U(A) \prod_{l \in \Pi} R_{l} e_{l} .
\end{aligned}
$$

[The case of $k=1$ ]

$$
\begin{aligned}
\mathfrak{O}(3 / 2, N, \chi)_{K}= & \sum_{\substack{0<B \mid M \\
B \neq M}} \sum_{\substack{0<A \mid(M / B) \\
\xi(\underline{A})=\chi}} V(4 B ; \xi)_{K} \mid \tilde{\delta}_{A} \\
& +\sum_{\substack{0<B \mid M \\
B \neq M}} \sum_{\substack{0<A \mid(M / B)^{2} \\
\xi(\underline{A})=\chi}} \sum_{\substack{0 \leq e_{l} \leq 2 \\
(l \in \Pi)}} V(4 B ; \xi)_{K} \quad U(A) \prod_{l \in \Pi} R_{l} e_{l} .
\end{aligned}
$$


Here, $\xi$ runs over all characters modulo $4 B$ such that $\xi(\underline{A})=\chi$.

$\mathfrak{O}(k+1 / 2, N, \chi)_{K}$ is a subspace of $S(k+1 / 2, N, \chi)_{K}$ if $k \geq 2$, resp. $V(N$; $\chi)_{K}$ if $k=1$. We denote by $\mathfrak{N}(k+1 / 2, N, \chi)_{K}$ the orthogonal complement of $\mathfrak{O}(k+1 / 2, N, \chi)_{K}$ in $S(k+1 / 2, N, \chi)_{K}$ resp. $V(N ; \chi)_{K}$ according as $k \geq 2$ resp. $k=1$.

Then the space $\mathfrak{N}(k+1 / 2, N, \chi)_{K}$ is stable by the twisting operators $R_{p}$ for all $p \in \Pi$. Hence we can decompose this space into common eigen subspaces as follows:

$$
\begin{gathered}
\mathfrak{N}(k+1 / 2, N, \chi)_{K} \\
=\bigoplus_{\kappa \in\{ \pm 1\}^{\Pi}} \mathfrak{N}^{\emptyset, \kappa}(k+1 / 2, N, \chi)_{K}, \\
\mathfrak{N}^{\emptyset, \kappa}(k+1 / 2, N, \chi)_{K} \\
\quad:=\left\{f \in \mathfrak{N}(k+1 / 2, N, \chi)_{K} ; f \mid R_{p}=\kappa(p) f \text { for all } p \in \Pi\right\} .
\end{gathered}
$$

We call these spaces $\mathfrak{N}^{\emptyset, \kappa}(k+1 / 2, N, \chi)_{K}$ the spaces of newforms of Kohnen space, because these subspaces $\mathfrak{N}^{\emptyset, \kappa}(k+1 / 2, N, \chi)_{K}\left(\kappa \in\{ \pm 1\}^{\Pi}\right)$ have the following nice properties.

(1) $\mathfrak{N}^{\emptyset, \kappa}(k+1 / 2, N, \chi)_{K}$ has an orthogonal $\mathbf{C}$-basis consisting of common eigenforms for all Hecke operators $\tilde{T}_{k+1 / 2, N, \chi}\left(p^{2}\right)(p$ : prime, $p \nmid M)$ and $U\left(p^{2}\right)$ ( $p$ : prime, $\left.p \mid M\right)$, which are uniquely determined up to multiplication with non-zero complex numbers. Let $f$ be such a common eigenform and $\lambda_{p}$ the eigenvalue of $f$ with respect to $\tilde{T}_{k+1 / 2, N, \chi}\left(p^{2}\right)(p \nmid M)$ resp. $U\left(p^{2}\right)(p \mid M)$, then there exists a primitive form $F \in S^{0}(2 k, M)$ of weight $2 k$ and of conductor $M$ which is uniquely determined and satisfies the following: For a prime $p$,

$$
F \mid T_{2 k, M}(p)=\lambda_{p} F \quad \text { if }(p, M)=1 \quad \text { and } \quad F \mid U(p)=\lambda_{p} F \quad \text { if } p \mid M .
$$

Here, we can find, by using the trace relation of Theorem 2 (1), which primitive form occurs via the above correspondence.

\section{(2) (The Strong Multiplicity One Theorem)}

Let $f, g$ be two non-zero elements of $\mathfrak{N}^{\emptyset, \kappa}(k+1 / 2, N, \chi)_{K}$. If $f$ and $g$ are common eigenforms of $\tilde{T}_{k+1 / 2, N, \chi}\left(p^{2}\right)$ with the same eigenvalue for all prime numbers $p$ prime to some integer $A$, then $\mathbf{C} f=\mathbf{C} g$.

Therefore $\mathfrak{N}^{\emptyset, \kappa}(k+1 / 2, N, \chi)_{K} \hookrightarrow S^{0}(2 k, M)$ as modules on the full Hecke algebra. 
(3) The space of oldforms $\mathfrak{O}(k+1 / 2, N, \chi)_{K}$ has also an orthogonal $\mathbf{C}$ basis consisting of common eigenforms for all Hecke operators $\tilde{T}_{k+1 / 2, N, \chi}\left(p^{2}\right)$ ( $p$ : prime, $p \nmid N)$. The system of eigenvalues of such a common eigenform corresponds to a primitive form of weight $2 k$ whose conductor is a divisor of $M$ and less than $M$ (cf. Theorem 3$)$.

(4) The space of oldform $\mathfrak{O}(k+1 / 2, N, \chi)_{K}$ is generated by the spaces of cusp forms of lower level. Hence, by induction, we see that the spaces $S(k+1 / 2, N, \chi)_{K}(k \geq 2)$ and $V(N ; \chi)_{K}(k=1)$ are reconstructed by the spaces of the types of $\mathfrak{N}^{\emptyset, \kappa}(k+1 / 2,4 B, \xi)_{K}$ and the operators of the types of $\tilde{\delta}_{A}, U(A)$, and $R_{l}$.

These operators $\tilde{\delta}_{A}, U(A)$, and $R_{l}$ (almost) fix the Fourier coefficients of cusp forms, i.e., for $f=\sum_{n \geq 1} a(n) \mathbf{e}(n z)$,

$$
\begin{aligned}
f \mid \tilde{\delta}_{A}(z) & :=A^{k / 2+1 / 4} f(A z)=A^{k / 2+1 / 4} \sum_{n \geq 1} a(n) \mathbf{e}(A n z), \\
f \mid U(A)(z) & :=\sum_{n \geq 1} a(A n) \mathbf{e}(n z), \\
f \mid R_{l}(z) & :=\sum_{n \geq 1} a(n)\left(\frac{n}{l}\right) \mathbf{e}(n z) .
\end{aligned}
$$

From this we claim the following: For studying Fourier coefficients of a cusp form in $S(k+1 / 2, N, \chi)_{K}$ resp. $V(N ; \chi)_{K}$, it is sufficient to study cusp forms only in the spaces of newforms $\mathfrak{N}^{\emptyset, \kappa}(k+1 / 2, N, \chi)_{K}$.

Remark. There exists a case such that $\mathfrak{N}^{\emptyset, \kappa}(k+1 / 2, N, \chi)_{K} \cong \mathfrak{N}^{\emptyset, \kappa^{\prime}}(k+$ $1 / 2, N, \chi)_{K}$ as modules over Hecke algebra for two distinct $\kappa, \kappa^{\prime} \in\{ \pm 1\}^{\Pi}$. For example we have the following isomorphism: Let $p$ be an odd prime and $\left\{\kappa, \kappa^{\prime}\right\}=\operatorname{Map}(\{p\},\{ \pm 1\})$. Then

$$
\begin{aligned}
& \mathfrak{N}^{\emptyset, \kappa}\left(k+1 / 2,4 p^{2}, \mathbf{1}\right)_{K} \cong \mathfrak{N}^{\emptyset, \kappa^{\prime}}\left(k+1 / 2,4 p^{2}, \mathbf{1}\right)_{K} \\
& \cong\left\{\bigoplus_{\sigma \in\{ \pm 1\}^{\{p\}}} S^{*(\mathbf{1}, \sigma)}\left(2 k, p^{2}\right)\right\} \\
& \oplus\left(1+\left(\frac{-1}{p}\right)\right) / 2\left\{S^{0}(2 k, p)\left|R_{p} \oplus S(2 k, \mathbf{1})\right| R_{p}\right\} .
\end{aligned}
$$

(cf. Theorem $2(1)$ ) 


\section{Appendix.}

We collect several propositions which are used in Section 2.

Let $k$ and $N$ be positive integers such that $4 \mid N$ and $\chi$ an even Dirichlet character modulo $N$ with $\chi^{2}=\mathbf{1}$.

Proposition A.1. Let $Q$ and a be two odd positive divisors of $N$ such that $(Q, N / Q)=(a, Q)=1$. For any $f \in S(k+1 / 2, N, \chi)$, the following identity holds good:

$$
f\left|U(a) \widetilde{W}(Q)=\chi_{Q}(a) f\right| \widetilde{W}(Q) U(a)
$$

where $\chi_{Q}$ is the $Q$-primary component of $\chi$.

Proof. If $Q=1$, the assertion is trivial and so we suppose that $Q \neq 1$.

We decompose $a=l_{1} l_{2} \cdots l_{t}\left(l_{1}, \ldots, l_{t}\right.$ are odd prime numbers $)$ and $Q=p_{1}{ }^{e_{1}} \cdots p_{n}{ }^{e_{n}}\left(p_{i}\right.$ 's are distinct prime numbers and $\left.e_{i} \geq 1\right)$.

First we consider the case of $t=1$ and will prove this case by using induction on $n$.

If $n=1$, the assertion follows from [U1, (1.20) (1)].

Suppose that $n \geq 2$ and the assertion holds good for any $m$ less than $n$.

Observing $f \mid U\left(l_{1}\right) \in S\left(k+1 / 2, N, \chi\left(\underline{l_{1}}\right)\right)$, we have the following formula from $[\mathrm{U} 1,(1.18)]$ and the assumption of the induction:

$$
\begin{aligned}
f \mid U\left(l_{1}\right) \widetilde{W}(Q)= & \left(\chi\left(\frac{l_{1}}{)}\right)\right)_{p_{n}}\left(Q p_{n}^{-e_{n}}\right) f \mid U\left(l_{1}\right) \widetilde{W}\left(Q p_{n}{ }^{-e_{n}}\right) \widetilde{W}\left(p_{n}{ }^{e_{n}}\right) \\
= & \chi_{p_{n}}\left(Q p_{n}^{-e_{n}}\right) \chi_{p_{1} \cdots p_{n-1}}\left(l_{1}\right) f \mid \widetilde{W}\left(Q p_{n}^{-e_{n}}\right) U\left(l_{1}\right) \widetilde{W}\left(p_{n}{ }^{e_{n}}\right) \\
= & \chi_{p_{n}}\left(Q p_{n}^{-e_{n}}\right) \chi_{p_{1} \cdots p_{n-1}}\left(l_{1}\right)\left(\chi\left(\frac{Q p_{n}{ }^{-e_{n}}}{p_{n}}\right)\right)_{p_{1}}\left(l_{1}\right) \\
& \times f \mid \widetilde{W}\left(Q p_{n}^{-e_{n}}\right) \widetilde{W}\left(p_{n}{ }^{e_{n}}\right) U\left(l_{1}\right) \\
= & \chi_{p_{n}}\left(Q p_{n}^{-e_{n}}\right) \chi_{p_{1} \cdots p_{n}}\left(l_{1}\right) f \mid \widetilde{W}\left(Q p_{n}^{-e_{n}}\right) \widetilde{W}\left(p_{n}{ }^{e}\right) U\left(l_{1}\right) \\
= & \chi_{p_{1} \cdots p_{n}}\left(l_{1}\right) f\left|\widetilde{W}(Q) U\left(l_{1}\right)=\chi_{Q}\left(l_{1}\right) f\right| \widetilde{W}(Q) U\left(l_{1}\right) .
\end{aligned}
$$

Next we suppose that $t \geq 2$ and the assertion holds good for any $s$ less than $t$. From the above formula and the assumption of the induction, we 
have the following:

$$
\begin{aligned}
f \mid U(a) \widetilde{W}(Q) & =f \mid U\left(l_{1} \cdots l_{t-1}\right) U\left(l_{t}\right) \widetilde{W}(Q) \\
& =\left(\chi\left(\frac{l_{1} \cdots l_{t-1}}{}\right)\right)_{Q}\left(l_{t}\right) f \mid U\left(l_{1} \cdots l_{t-1}\right) \widetilde{W}(Q) U\left(l_{t}\right) \\
& =\chi_{Q}\left(l_{t}\right) \chi_{Q}\left(l_{1} \cdots l_{t-1}\right) f \mid \widetilde{W}(Q) U\left(l_{1} \cdots l_{t-1}\right) U\left(l_{t}\right) \\
& =\chi_{Q}(a) f \mid \widetilde{W}(Q) U(a) .
\end{aligned}
$$

Thus we obtain the assertion.

Now, we choose and fix an odd prime number $p$ satisfying $(p, N)=1$. Take a form $g \in S(k+1 / 2, N, \chi)$. Then $g$ is also contained in $S(k+$ $1 / 2, N p, \chi)$. Hence we can consider the form $g \mid Y_{p}$ (See [U1, p. 155] for the definition of $Y_{p}$ ).

Since $(p, N)=1$ and $\chi$ is defined modulo $N$, we have $\chi_{p}=\mathbf{1}$. Therefore from $[\mathrm{U} 1,(1.27)], p^{-k+3 / 2} g|U(p) \widetilde{W}(p) U(p) \widetilde{W}(p)=g| Y_{p}^{2}=\left(\frac{-1}{p}\right) p g$.

Using $[\mathrm{U} 1,(1.18)], p^{-k+3 / 2} g\left|U(p) \widetilde{W}(p) U(p)=\left(\frac{-1}{p}\right) p g\right| \widetilde{W}(p)^{-1}=$ $\left(\frac{-1}{p}\right) p\left(\frac{-1}{p}\right)^{k+1 / 2} \chi_{p}(-1) \chi_{N}(p) g \mid \widetilde{W}(p)$.

Since $g \in S(k+1 / 2, N, \chi)$ and $\widetilde{W}(p)=\gamma_{p}{ }^{*} \tilde{\delta}_{p}$ for some $\gamma_{p} \in \Gamma(N)$, we get $g|\widetilde{W}(p)=g| \tilde{\delta}_{p}$.

Thus we obtain the following formula for any $g \in S(k+1 / 2, N, \chi)$.

$$
g\left|\tilde{\delta}_{p}=\left(\frac{-1}{p}\right)^{k+1 / 2} \chi(p) p^{-k / 2-1 / 4} g\right| Y_{p} U(p) .
$$

Next we consider $g=\sum_{n=1}^{\infty} a(n) \mathbf{e}(n z) \in S(k+1 / 2, N, \chi)$ as an element of $S(k+1 / 2, N p, \chi)$ and apply [U1, (1.26), (1.18)] to $g$.

$$
\begin{aligned}
g \mid Y_{p}= & \left(\frac{-1}{p}\right)^{k+3 / 2} \chi(p) g \mid \widetilde{W}(p) \tilde{\delta}_{p}+\left(\frac{-1}{p}\right)^{-1 / 2} p^{1 / 2} \sum_{n \geq 1} a(n)\left(\frac{n}{p}\right) \mathbf{e}(n z) \\
= & \left(\frac{-1}{p}\right)^{k+3 / 2} \chi(p) g \mid \tilde{\delta}_{p^{2}}+\left(\frac{-1}{p}\right)^{-1 / 2} p^{1 / 2} \sum_{n \geq 1} a(n)\left(\frac{n}{p}\right) \mathbf{e}(n z) \\
= & \left(\frac{-1}{p}\right)^{k-1 / 2} \chi(p) p^{3 / 2-k} \\
& \times \sum_{n \geq 1}\left\{p^{2 k-1} a\left(n / p^{2}\right)+p^{k-1} \chi(p)\left(\frac{-1}{p}\right)^{k}\left(\frac{n}{p}\right) a(n)\right\} \mathbf{e}(n z),
\end{aligned}
$$


where $a\left(n / p^{2}\right)=0$ if $p^{2} \not n$.

By using [Sh1, Theorem (1.7)], this formula is expressed by $U\left(p^{2}\right)$ and the Hecke operator $\tilde{T}_{k+1 / 2, N, \chi}\left(p^{2}\right)$, i.e., for any $g \in S(k+1 / 2, N, \chi)$,

$$
g \mid Y_{p}=\left(\frac{-1}{p}\right)^{k-1 / 2} \chi(p) p^{3 / 2-k}\left(g\left|\tilde{T}_{k+1 / 2, N, \chi}\left(p^{2}\right)-g\right| U\left(p^{2}\right)\right) .
$$

Combining (A.2) with (A.3), we have for any $g \in S(k+1 / 2, N, \chi)$,
$g|\widetilde{W}(p)=g| \tilde{\delta}_{p}=p^{-3 k / 2+5 / 4}\left(g\left|\tilde{T}_{k+1 / 2, N, \chi}\left(p^{2}\right) U(p)-g\right| U\left(p^{3}\right)\right)$.

Now we assume moreover that $g$ is an eigen form of $\tilde{T}\left(p^{2}\right)$, i.e.,

$$
g \mid \tilde{T}_{k+1 / 2, N, \chi}\left(p^{2}\right)=\lambda_{p} g \quad\left(\lambda_{p} \in \mathbf{C}\right) .
$$

and let $u$ be a squarefree odd positive integer such that $\left(u, 4 M_{2+}\right)=1$ and $\chi_{u}=\mathbf{1}$. Here, $\chi_{u}$ is the $u$-primary component of $\chi$.

We will describe the action of $Y_{p}$ on the forms $f:=g \mid U(u)$ and $f\left|U\left(p^{2}\right)=g\right| U\left(u p^{2}\right)$. Here, we consider the action of $Y_{p}$ on the space $S(k+1 / 2, \tilde{N}, \chi(\underline{u}))(\tilde{N}$ is the least common multiple of $N p$ and $u)$.

[Case 1] First we assume that $(p, u)=1$.

Since $g \mid U(p)$ is contained in $S(k+1 / 2, \tilde{N}, \chi(\underline{p}))$, we can apply Proposition A.1 to $g \mid U(p)$. Hence we have $f\left|Y_{p}=p^{-k / 2+3 / 4} g\right| U(p) U(u)$ $\widetilde{W}(p)=\left(\frac{u}{p}\right) p^{-k / 2+3 / 4} g\left|U(p) \widetilde{W}(p) U(u)=\left(\frac{u}{p}\right) g\right| Y_{p} U(u)$.

By using (A.3) and (A.5),

$$
f \mid Y_{p}=\left(\frac{u}{p}\right)\left(\frac{-1}{p}\right)^{k-1 / 2} \chi(p) p^{3 / 2-k}\left(\lambda_{p} f-f \mid U\left(p^{2}\right)\right) .
$$

Applying $Y_{p}$ to the both sides of the above formula and using $[\mathrm{U} 1,(1.27)]$,

$$
\left(\frac{-1}{p}\right) p f=f \mid Y_{p}^{2}=\left(\frac{u}{p}\right)\left(\frac{-1}{p}\right)^{k-1 / 2} \chi(p) p^{3 / 2-k}\left(\lambda_{p} f\left|Y_{p}-f\right| U\left(p^{2}\right) Y_{p}\right) .
$$

Modifying this formula and using (A.6), we have

$$
\begin{aligned}
f \mid U\left(p^{2}\right) Y_{p} & =-\left(\frac{u}{p}\right)\left(\frac{-1}{p}\right)^{-k+3 / 2} \chi(p) p^{k-1 / 2} f+\lambda_{p} f \mid Y_{p} \\
& =\left(\frac{u}{p}\right)\left(\frac{-1}{p}\right)^{k-1 / 2} \chi(p) p^{3 / 2-k}\left\{\left(\lambda_{p}^{2}-p^{2 k-2}\right) f-\lambda_{p} f \mid U\left(p^{2}\right)\right\} .
\end{aligned}
$$


We can represent these relations as a matrix as follows:

$$
\begin{aligned}
& \left(f\left|Y_{p}, f\right| U\left(p^{2}\right) Y_{p}\right) \\
& =\left(f, f \mid U\left(p^{2}\right)\right) \\
& \quad \times\left(\frac{u}{p}\right)\left(\frac{-1}{p}\right)^{k-1 / 2} \chi(p) p^{3 / 2-k}\left(\begin{array}{cc}
\lambda_{p} & \lambda_{p}^{2}-p^{2 k-2} \\
-1 & -\lambda_{p}
\end{array}\right) .
\end{aligned}
$$

The characteristic polynomial of this matrix is $t^{2}-\left(\frac{-1}{p}\right) p$. Hence, this matrix has two distinct eigen values.

[Case 2] Next we assume that $p \mid u$.

Since $g \mid U\left(p^{2}\right)$ is contained in $S(k+1 / 2, \tilde{N}, \chi)$, we can apply Proposition A.1 to $g \mid U\left(p^{2}\right)$. Observing $\chi_{p}=\mathbf{1}, f\left|Y_{p}=p^{-k / 2+3 / 4} g\right| U\left(p^{2}\right) U(u / p)$ $\widetilde{W}(p)=p^{-k / 2+3 / 4} g \mid U\left(p^{2}\right) \widetilde{W}(p) U(u / p)$.

By using (A.3) and (A.5), $g\left|U\left(p^{2}\right)=\lambda_{p} g-\left(\frac{-1}{p}\right)^{-k+1 / 2} \chi(p) p^{k-3 / 2} g\right|$ $Y_{p}$. Applying $\widetilde{W}(p)$ to the both sides,

$$
g\left|U\left(p^{2}\right) \widetilde{W}(p)=\lambda_{p} g\right| \widetilde{W}(p)-\left(\frac{-1}{p}\right)^{-k+1 / 2} \chi(p) p^{k-3 / 2} g \mid Y_{p} \widetilde{W}(p)
$$

Observing $g \mid U(p) \in S(k+1 / 2, \tilde{N}, \chi(\underline{p}))$, we apply [U1, (1.18)] to $g \mid$ $U(p)$,

$$
\begin{aligned}
g \mid Y_{p} \widetilde{W}(p) & =p^{-k / 2+3 / 4} g \mid U(p) \widetilde{W}(p)^{2} \\
& =p^{-k / 2+3 / 4}\left(\frac{-1}{p}\right)^{k-1 / 2} \chi(p) g \mid U(p) .
\end{aligned}
$$

Combining (A.4), (A.8), and (A.9), we have

$$
p^{-k / 2+3 / 4} g \mid U\left(p^{2}\right) \widetilde{W}(p)=p^{-2 k+2}\left\{\left(\lambda_{p}^{2}-p^{2 k-2}\right) g\left|U(p)-\lambda_{p} g\right| U\left(p^{3}\right)\right\}
$$

Therefore

$$
f \mid Y_{p}=p^{-2 k+2}\left\{\left(\lambda_{p}^{2}-p^{2 k-2}\right) f-\lambda_{p} f \mid U\left(p^{2}\right)\right\} .
$$

Applying $Y_{p}$ to the both sides of the above (cf. [U1, (1.27)]), $(p-1) f\left|Y_{p}+p f=f\right| Y_{p}^{2}=p^{-2 k+2}\left\{\left(\lambda_{p}^{2}-p^{2 k-2}\right) f\left|Y_{p}-\lambda_{p} f\right| U\left(p^{2}\right) Y_{p}\right\}$. 
Modifying this formula and using (A.10),

$$
\begin{aligned}
& f \mid U\left(p^{2}\right) Y_{p} \\
& =p^{-2 k+2}\left\{\left(\lambda_{p}^{3}-\lambda_{p}\left(p^{2 k-1}+p^{2 k-2}\right)\right) f-\left(\lambda_{p}{ }^{2}-p^{2 k-1}\right) f \mid U\left(p^{2}\right)\right\} .
\end{aligned}
$$

We can represent these relations as a matrix as follows:

$$
\begin{aligned}
(\mathrm{A} .11)= & \left(f, f \mid U\left(p^{2}\right)\right) \\
& \times p^{-2 k+2}\left(\begin{array}{cc}
\lambda_{p}{ }^{2}-p^{2 k-2} & \lambda_{p}{ }^{3}-\lambda_{p}\left(p^{2 k-1}+p^{2 k-2}\right) \\
-\lambda_{p} & -\left(\lambda_{p}^{2}-p^{2 k-1}\right)
\end{array}\right) .
\end{aligned}
$$

The characteristic polynomial of this matrix is $t^{2}-(p-1) t-p=$ $(t-p)(t+1)$. Hence, this matrix has two distinct eigen values.

\section{REFERENCES}

[K] W. Kohnen, Newforms of half-integral weight, J. reine und angew. Math., 333 (1982), 32-72.

[M] T. Miyake, Modular Forms, Springer, 1989.

[M-R-V] Manickam, Ramakrishnan, and Vasudevan, On the theory of Newforms of half-integral weight, J. of Number theory, 34 (1990), 210-224.

[N] S. Niwa, On Shimura's trace formula, Nagoya Math. J., 66 (1977), 183-202.

[S] J.-P. Serre, Linear representations of finite groups, G. T. M. series, 42, Springer, 1977.

[Sh1] G. Shimura, On modular forms of half integral weight, Ann. of Math., 97 (1973), 440-481.

[Sh2] G. Shimura, The critical values of certain zeta functions associated with modular forms of half-integral weight, J. Math. Soc. Japan, 33-4 (1981), 649-672.

[Sh3] G. Shimura, Introduction to the arithmetic theory of automorphic functions, Publ. Math. Soc. Japan, 11 (1971), Iwanami Shoten and Princeton Univ. Press.

[She] T. R. Shemanske, Cuspidal Newforms and Character twists, J. reine angew. Math., 328 (1981), 58-71.

[She-W] T. R. Shemanske and L. H. Walling, Determining multiplicities of Half-integral weight newforms, Pacific J. of Math., 167 (1995), 345-383.

[U1] M. Ueda, On twisting operators and newforms of half-integral weight, Nagoya Math. J., 131 (1993), 135-205.

[U2] M. Ueda, On Representations of Finite Groups in the Space of Modular Forms of Half-integral Weight, Proc. of The Japan Acad., 70 (1994), 198-203.

[U3] M. Ueda, The trace formulae of twisting operators on the spaces of cusp forms of half-integral weight and some trace relations, Japanese J. of Math., 17-1 (1991), 83-135. 
[U4] M. Ueda, The decomposition of the spaces of cusp forms of half-integral weight and trace formula of Hecke operators, J. Math. Kyoto Univ., 28 (1988), 505-555.

[U5] M. Ueda, Supplement to the decomposition of the spaces of cusp forms of half-integral weight and trace formula of Hecke operators, J. Math. Kyoto Univ., 31 (1991), 307-309.

[U6] M. Ueda, in preparation.

Department of Mathematics

Faculty of Science

Nara Women's University

Nara 630 Japan

m-ueda@cc.nara-wu.ac.jp 